\title{
Diagnostic evaluation of smear negative tuberculosis in a resource poor setting
}

Citation for published version (APA):

Otieno, M. G. (2017). Diagnostic evaluation of smear negative tuberculosis in a resource poor setting. [Doctoral Thesis, Maastricht University]. Datawyse / Universitaire Pers Maastricht. https://doi.org/10.26481/dis.20170322gom

Document status and date:

Published: 01/01/2017

DOI:

10.26481/dis.20170322gom

Document Version:

Publisher's PDF, also known as Version of record

Document license:

Unspecified

\section{Please check the document version of this publication:}

- A submitted manuscript is the version of the article upon submission and before peer-review. There can be important differences between the submitted version and the official published version of record.

People interested in the research are advised to contact the author for the final version of the publication, or visit the DOI to the publisher's website.

- The final author version and the galley proof are versions of the publication after peer review.

- The final published version features the final layout of the paper including the volume, issue and page numbers.

Link to publication

\footnotetext{
General rights rights.

- You may freely distribute the URL identifying the publication in the public portal. please follow below link for the End User Agreement:

www.umlib.nl/taverne-license

Take down policy

If you believe that this document breaches copyright please contact us at:

repository@maastrichtuniversity.nl

providing details and we will investigate your claim.
}

Copyright and moral rights for the publications made accessible in the public portal are retained by the authors and/or other copyright owners and it is a condition of accessing publications that users recognise and abide by the legal requirements associated with these

- Users may download and print one copy of any publication from the public portal for the purpose of private study or research.

- You may not further distribute the material or use it for any profit-making activity or commercial gain

If the publication is distributed under the terms of Article $25 \mathrm{fa}$ of the Dutch Copyright Act, indicated by the "Taverne" license above, 


\section{Diagnostic Evaluation of Smear Negative}

Tuberculosis in a Resource Poor Setting

\section{Mala George Otieno}




\section{Du Mastricht University in Leaning!}

(C) Mala George Otieno, Maastricht2016

No part of this book may be reproduced or transmitted in any for morby any means, without prior permission in writing by the author, or when appropriate, by the publisher of the publication

Coverpicture Irvin Talboom

ISBN 9789461596703

Production print Datawyse | Universitaire Pers Maastricht 


\title{
Diagnostic Evaluation of Smear Negative Tuberculosis in a Resource Poor Setting
}

\author{
Dissertation \\ to obtain the degree of Doctorate Maastricht University, \\ on the authority of the Rector Magnificus, Prof.dr.Rianne M. Letschert \\ in accordance with the decision of Board of Deans, \\ to be defended in public on \\ Wednesday, 22 March 2017 at 14.00 hours
}

by

Mala George Otieno 


\section{Promoter}

Prof. dr. G.J. Dinant

\section{Co-promoter}

Dr. M.G. Spigt

\section{Assessment committee}

Prof. dr. J.F.C. Glatz (chair)

Prof. Dr. G.J. Wesseling

Dr. C.D.J. den Heijer

Dr. P. van den Hombergh, AMC Amsterdam

Dr. M.A. Mahmud, Mekelle University, Ethiopia

\section{Financial enclosure}

The research presented in this thesis was conducted at the School for Public Health and Primary Care: CAPHRI, Department of Family Medicine, of Maastricht University. CAPHRI participates in the Netherlands School of Primary Care Research CaRe. The research was supported fully by The Netherlands Fellowship Programs (NFP) that promote capacity building within organizations in 51 countries by providing fellowships for training and education for professionals. The NFP is initiated and fully funded by the Dutch Ministry of Foreign Affairs from the budget for development cooperation. 


\section{Contents}

Abbreviations

Chapter 1 General introduction

Chapter 2 Quality of diagnosis and monitoring of tuberculosis in Northern

Ethiopia: medical records-based retrospective study

Tropical Doctor 2015; 45(4):214-20

Chapter 3 Why tuberculosis service providers do not follow treatment guideline in Ethiopia: a qualitative study

Journal of Evaluation in Clinical Practice 2014; 20(1):88-93

Chapter 4 Predicting tuberculosis in clinical suspects and among smear negative cases, with routinely available diagnostic tools in Ethiopia Submitted

Chapter 5 Exploratory analyses of the level of salivary Nitric Oxide (NO) metabolites and microbiological outcome in suspected Tuberculosis patients

Submitted

Chapter 6 General Discussion

Summary

Valorisation

Acknowledgements

Biography 


\section{Abbreviations}

$\begin{array}{ll}\text { AFB } & \text { Acid Fast Bacilli } \\ \text { AUC } & \text { Area Under Curve } \\ \text { BMI } & \text { Body Mass Index } \\ \text { CT } & \text { Computer Tomography } \\ \text { CXR } & \text { Chest X-Ray } \\ \text { DNA } & \text { Deoxyribonucleic Acid } \\ \text { DOTS } & \text { Directly Observed Treatment-short Course } \\ \text { DST } & \text { Drug Susceptibility Testing } \\ \text { FDCS } & \text { Fixed Drug Combinations } \\ \text { FGDs } & \text { Focus Group Discussions } \\ \text { HIV } & \text { Human Immunodeficiency Virus } \\ \text { HRCT-scan } & \text { High Resolution Computer Tomography-scan } \\ \text { IFN-y } & \text { Interferon-gamma } \\ \text { ISTC } & \text { International Standards for Tuberculosis Care } \\ \text { LMICS } & \text { Low-and Middle-Income Countries } \\ \text { MDR-TB } & \text { Multidrug Resistant-TB } \\ \text { MGIT } & \text { Mycobacterium Growth Indicator Tube } \\ \text { MTB/RIF } & \text { New molecular test for detecting the presence of Mycobacterium TB } \\ \text { NO } & \text { Nitric Oxide } \\ \text { ORs } & \text { Odds Ratios } \\ \text { PCR } & \text { Polymerase Chain Reaction } \\ \text { QFT test } & \text { Immunological test for detecting the presence of Mycobacterium TB } \\ \text { RHZ } & \text { Rifampicin, Isoniazid and Pyrazinamide } \\ \text { RHZE } & \text { Rifampicin, Isoniazid, Pyrazinamide and Ethambutanol } \\ \text { RNS } & \text { Reactive Nitrogen Species } \\ \text { ROC curve } & \text { Receiver Operating Characteristic curve } \\ \text { TB } & \text { Tuberculosis } \\ \text { WHO } & \text { World Health Organization } \\ & \end{array}$


Chapter

1

General introduction 



\subsection{INTRODUCTION AND RESEARCH SCOPE}

The 22 high-burden Tuberculosis (TB) countries account for 95\% of world's TB cases and $98 \%$ of TB related deaths [1].Only 14 of these countries reached the $85 \%$ target for treatment success among all new cases in 2010 [2]. This despite the availability of highly effective TB therapy that could cure $90 \%$ of cases, and an intensive worldwide effort to improve access to the diagnostic and treatment interventions embodied in the directly observed treatment-short course (DOTS) strategy. Apart from that, in Ethiopia smearpositive case detection rate remains at 34\%, far less than the World Health Organization (WHO) estimate of 70\% [3]. The regional disaggregation showed highest case detection rate in urban administrations (Harari 95\%, Dire Dawa $81 \%$ and Addis Ababa 63\%); whereas, Somali, Amhara and Tigray regions performed low at 19\%, 23\%, and $26 \%$ respectively. Furthermore, the performance of the diagnostic and treatment guideline is far from good in real life [4], as it is often negatively influenced by local factors and practice related context $[5,6]$. Consequently, the relative importance of the different characteristics of a diagnostic test depends upon the setting in which the test is to be performed and the intended use of the results. Hence, the focus for TB control has moved towards reaching successful treatment of all cases of TB through offering a standard of care consistent with TB guidelines [7]. However, recent studies have reported multiple pitfalls in the diagnosis and treatment of TB patients, even in regions where TB might be considered a rarity [8-10]. These pitfalls echo a fundamental principle of TB control espoused by Piot more than 40 years ago, that a substantial proportion of TB cases occur among individuals who fail to access high-quality diagnostic and treatment services [11]. Therefore, this research will seek to offer real-life examples of what is needed to customize TB diagnostic guidelines to local contexts of healthcare in order to improve compliance.

\subsection{BACKGROUND}

\subsubsection{Global quality of TB care}

TB remains a major global health problem. In 2012 an estimated 8.6 million people developed the disease, and 1.3 million people died [12]. Great progress has been made in combating TB. The 2015 Millennium Development Goal of halting and beginning to reverse the incidence of TB has been achieved, and a target of $50 \%$ reduction in mortality compared with 1990 is on track. However, in some parts of the world, like Africa, this target may not be reached, and the global elimination target set for 2050 does not currently seem within reach, because TB incidence is declining far too slowly. Current TB control strategies in many countries are crucially dependent on care that is provided to persons who have symptoms of TB and those who have the disease. When TB is not 
considered and appropriately evaluated in persons with early symptoms, the resulting delay promotes further transmission of infection and more severe disease [13, 14].

Thus, individual care of people with TB symptoms or disease is the key and, at the same time, the vulnerable point of current public health actions for TB control. Substantial variations in the clinical approach of persons with TB symptoms or disease have been observed within and among countries, in the public and private health care sector, and among clinicians in the same health facility $[15,16]$. These variations can have important implications for TB control. While many national TB control programs have progressively adopted the recommendations for TB care of the World Health Organization (WHO), private practitioners, non-governmental caregivers, and academics often have perceived national TB control program guidelines as inferior or not applicable to their settings. To address these concerns and ensure maximum adherence to scientific evidence, an international coalition of organizations led by the WHO and the American Thoracic Society published the first edition of the International Standards for TB Care (ISTC) in 2006. The ISTC aims to unify approaches to clinical care of persons with TB symptoms or disease, irrespective of the country or setting in which care is taking place [17].

\subsubsection{TB burden in Ethiopia}

Ethiopia is one of the world's high TB, TB/HIV and multi-drug resistant TB (MDR-TB) burden countries. Prevalence and incidence of TB are 210 and 224 per 100000 persons respectively [18]. The estimated TB case detection rate (percentage of detected cases among the estimated number of incident cases) in Ethiopia has been consistently low; the WHO estimate is $64 \%$ for all forms. According to the same WHO report, there were 200000 prevalent TB cases in Ethiopia in 2013 (211 per 100 000). TB mortality is estimated at 30000 deaths (excluding deaths among HIV positive TB cases) or 32 deaths per 100000 per year. In a recent Ethiopian national population based survey in 2011, the prevalence of bacteriologically-confirmed TB (smear and/or culture positive) for persons aged 15 years and above was 277 per 100,000 [ 19 ]. The prevalence of smear positive pulmonary TB was 108 per 100000, or 63 per 100000, including children. The population-based TB survey concluded that TB burden in Ethiopia was lower than previously thought, which may indicate better program performance. However, a high proportion of TB among young persons' suggests that TB is circulating in the community and that there is a need for more efforts to limit the spread of TB disease [20].

Both men and women are equally vulnerable to TB in their productive ages of 20-30 years. Gender differences in health-seeking behaviour might contribute to differences in delay in TB diagnosis, treatment interruption, gender based TB stigma and discrimination [21]. Low detection rate and treatment for TB among women have been seen due to delay in accessing care, poor compliance to treatment, and belief in alternate treatments [21]. The reporting system does not disaggregate data by sex, but a recent study 
found no difference in diagnostic delay and treatment by sex [21]. The delay in males was higher but not statistically different.

\subsubsection{TB diagnostics}

In its Global TB Report 2013, the WHO highlighted detection of missed cases as a priority action to reach 2015 global targets [22]. The report notes "TB remains unique among the major infectious diseases in lacking accurate and rapid point-of-care tests, largely due to insufficient progress in biomarker discovery for TB diagnosis. The most pressing priority in TB diagnostics research today is the development of a simple, low-cost, instrument-free rapid test of suspected patients". Currently, the diagnosis relies on demonstration of the presence of bacteria in clinical specimens by serial sputum smear microscopy and culture. When applied to resource-limited settings, these methods suffer from significant limitations. Serial sputum smear microscopy requires the patient to make repeated trips to the clinic, which often is cost-prohibitive for the patient, resulting in the inability to properly diagnose and treat the patient. In addition, TB diagnostic tests are either too expensive, too time consuming (24 hour or more), or require specialized equipment, expertise, and power [23-25]. Furthermore, diagnostic tests have low sensitivities (50\% in the sputum smear test), or are unreliable when used on HIV-positive patients or children [26-28].

\subsubsection{Providers adherence to diagnostic guidelines}

Substantial effort has been devoted to improving physician compliance with evidencebased guidelines. Physicians are under increasing pressure to comply with evidencebased guidelines and to achieve high performance measures. Nevertheless, compliance with so-called best practices is far from ideal [29-31]. The barriers to guideline compliance have been categorized into three domains: physician knowledge (lack of awareness, lack of familiarity, or oversight), physician attitudes (lack of agreement; lack of self-efficacy that is, the belief that a physician can perform guideline recommendations; lack of outcome expectancy that is, skepticism that complying with the guideline would help patients; or the inertia of previous practice), and external barriers [32].

Studies of the quality of TB evaluation (i.e. diagnostic workup of patients with symptoms suggestive of TB) in high burden countries have generally shown poor adherence to international or national guidelines. ISTC, Edition 3 TB CARE I, The Hague, 2014, which has been endorsed by nearly all TB programs worldwide, recommended that at a minimum, all patients in high burden countries with cough of at least 2 weeks' duration should have at least two sputum smears examined for acid-fast bacilli (AFB) and be treated for TB if sputum AFB is smear-positive. Previous studies found that providers are not following guidelines for TB diagnosis and treatment, both from the public and private sector in many other high burden countries [33-39]. While previous research, 
including quantitative [40-42], qualitative [43-49] and mixed methods approaches [50], has assessed barriers patients face in accessing primary care centers that provide TB diagnostic services, less is known about barriers providers in these settings face in adhering to guidelines for evaluating patients for TB. The two studies evaluating provider perspectives have focused on their perceptions about patient barriers to accessing TB care $[43,51]$. Less well understood are the determinants of provider adherence to TB guidelines. In particular, there is increasing recognition that guideline implementation is heavily dependent on provider behaviour and in order to improve the quality of care, understanding and subsequently changing provider behaviour is required.

\subsubsection{Pathway to better diagnostics}

Poor diagnosis remains a major obstacle to global TB control. In most high-burden countries, TB is still diagnosed using tools such as direct sputum microscopy and chest radiographs. Although a large number of systematic reviews have been published on TB diagnostics, almost all focus on test accuracy (i.e. sensitivity and specificity) [52]. Evidence on test accuracy is essential, but policy development requires more than that. Along with data on test accuracy, there is need to consider user-important as well as patient-important outcomes $[53,54]$. User-important outcomes consist of practical concerns for the usability of a test in real-practice situations. Although these generally do not require fundamentally different strategies to evaluate, it is important that they are assessed under implementation or real-live settings. Wherein a study shows that implementing a diagnostic test in a given situation results in clinically relevant improvements in patient care and patient outcomes. For TB diagnostics, this might mean an increased number of patients detected and receiving appropriate treatment, fewer patients defaulting from the diagnostic pathway due to reduced numbers of patient visits, or more patients cured due to accurate detection of drug resistance [44, 52]. These types of evidence must be taken into account, along with test accuracy and reliability, when policy makers or programs are evaluating a diagnostic test or procedure for recommendation or widespread use [50]. Systematic reviews of diagnostics should make an effort to summarize data on these outcomes in addition to accuracy, appraise the quality of available evidence, and explore the uncertainty regarding the often assumed values and preferences of patients associated with these tests.

\subsubsection{Predictive model for diagnosis of TB}

Predictive models for the diagnosis of TB provide a useful framework for systematization of the diagnostic approach and are able to standardize data collection from clinicians [55]. Numerous decisions are made by care providers, on the basis of an estimated probability that a specific disease or condition is present (diagnostic setting) in an individual. In the diagnostic setting, the probability that a particular disease is present 
can be used, for example, to inform the referral of patients for further testing, to initiate treatment directly, or to reassure patients that a serious cause for their symptoms is unlikely. In diagnostic settings, probability estimates are commonly based on combining information from multiple predictors observed or measured from an individual $[56,57]$. Information from a single predictor is often insufficient to provide reliable estimates of diagnostic probabilities or risks [58]. In virtually all medical domains, diagnostic multivariable (risk) prediction models are being developed, validated, updated, and implemented with the aim to assist doctors and individuals in estimating probabilities and potentially influence their decision making. These models could be used to identify patients at very high risk of TB that may require further diagnostic tests after the results of a negative smear test. Besides, further research is necessary to determine the usefulness of these prediction models to establish the exact causes of false smear negative TB (patients that have TB, but have a negative smear test), that are still unknown. These models are suited to resource-poor high TB burden settings, where the available diagnostic test is perceived to be poor and some variables may have lost their predictive value.

\subsubsection{Clinical need for biomarkers of extent of tuberculosis}

Biomarkers are measurable characteristics that indicate normal biological or pathogenic processes, or pharmacological responses to a therapeutic intervention [59]. It has been suggested that volatile metabolites from Mycobacterium tuberculosis ( $M$. tuberculosis) organisms may be exploited to diagnose TB rapidly and could be used as biomarkers of cure and relapse [60]. However, the metabolite pattern found in individuals will vary greatly depending on the time of the day when the blood or urine sample is taken, and food, fluid or drug intake before sample collection, etc. For clinical trials, standardization of patients will become crucial. Although no published results exist for TB biomarker discovery using advanced technological platforms, metabolomics have been used to analyse several other conditions [61] and there is good reason to believe that similar approaches might be of benefit in TB biomarker discovery. Hosts as well as mycobacterial metabolites might be able to provide insight into host-pathogen specific interactions as well as disease state and treatment response and might be able to predict final outcome. This is an area of much interest and may lead to a practical, noninvasive way of diagnosing TB at point of care. However, there is no evidence yet that these markers can predict microbiological and treatment outcome.

\subsubsection{Nitric oxide in TB}

Nitric oxide (NO) is a signaling molecule with several well-known biological functions including vasodilation, neurotransmission and immune response [62].Several animal and macrophage experiments have shown that $\mathrm{NO}$ and related reactive nitrogen spe- 
cies (RNS) constitute a major host defense mechanism against intracellular pathogens including $M$. tuberculosis, in both the acute and the latent phases of infection $[63,64]$. It has also been shown that RNS are actively produced in human TB, although their relative importance is controversial [65-70]. Thus, uncertainties exist for the use of NO to diagnose TB prior to initiation of therapy, the most important being the degree to which this biomarker can differentiate recent or progressive from latent infection [71]. Inability to distinguish these two states will greatly reduce its potential to add value to clinical decision-making in TB-endemic regions, where a majority of adults would be expected to have been exposed to $M$. tuberculosis. There are many studies that suggest that the magnitude of the immune response reflects the magnitude of the bacterial load and two longitudinal studies in untreated contacts suggest that the highest responders have the highest risk of TB $[72,73]$, but these studies are relatively small and lack detailed sequential testing routines. Thus the kinetics and duration of the differences are unknown; however, the fact that the two studies produced similar results in very different populations and environments (Ethiopia and Germany) is encouraging. Studies to address the questions of remote versus recent infection, predictive value and optimization of the assays, should prove very valuable.

\subsection{OBJECTIVES AND OUTLINE OF THE THESIS}

The studies presented in this thesis focus on the quality of diagnostic care provided to suspected TB patients and centre on four principle investigations. The central aim of the studies presented in Chapters 2 and 3 was to improve understating of the current quality of diagnostic care and reasons for not following the guidelines. To achieve this, in Chapter 2 we first determined the quality of diagnostic care offered to patients with smearnegative TB compared with smear-positive cases. We primarily evaluated clinical measures commended by ISTC which represent activities that TB care providers perform mostly themselves and are consistent with the national Ethiopian TB guideline. While, in Chapter 3 we explored health care providers' practice-related and contextspecific reasons for non-compliance to diagnostic procedures at health facilities in Ethiopia. Our findings may offer real-life examples of what is needed to customize TB guidelines to local contexts of health care in order to improve compliance. We also examined whether a barrier is actual or perceived, as this may also affect the type of intervention needed to overcome it. The overarching aim of the study presented in Chapter 4 is to develop strategies to diagnose TB in patients whose initial test is negative for smears. These cases are mostly culture-negative TB and their diagnosis is problematic. Therefore, a prospective study was designed to determine the predictors of culture-negative TB among smear negative TB cases. Finally, in the Global TB Report 2014, the WHO declared that "one of the most urgently needed tests is a rapid biomarker-based test that can diagnose TB". Ideally, such TB biomarker should be able to detect the earliest 
and most difficult to diagnose TB cases, and should be suitable for use in resourcelimited settings. Although previous studies show that the presence of NO and its metabolites are potential screening biomarkers for TB, its relative importance is debated. Therefore, there is very limited evidence on NO in direct diagnosis of TB and in addition to lack of clinical studies describing the level of salivary NO in suspected TB patients. So, in Chapter 5, we evaluated the role of salivary NO in TB diagnosis.

These above aims raise the following study objectives:

1. To determine quality of diagnosis and monitoring of treatment response of patients with smear-negative TB compared with smear-positive cases in Ethiopia.

2. To explore TB service providers' reasons for not complying with TB treatment guidelines.

3. To evaluate the performance of predictors for estimating the probability of tuberculosis (TB) (1) among all clinical suspects and (2) among smear negative cases.

4. To assess the diagnostic value of the level salivary NO metabolites with microbiological outcome (infectiousness), and its association with the severity of the disease (commonly seen as wasting) in TB suspects.

Finally, we consider the main findings, methodological issues and general conclusion in a general discussion chapter. We conclude with a brief summary of the overall findings of each study presented in this thesis and implications, both for the theoretical development of research on TB care and specific public policies for professional practice. 


\section{REFERENCE}

1. Raviglione MC, Gupta R, Dye CM, Espinal MA: The burden of drug-resistant TB and mechanisms for its control. Ann N Y Acad Sci 2001, 953:88 - 97.

2. WHO. Global TB Report. WHO 2012.

3. FMOH, March 2010, TB prevention and control program Annual Bulletin

4. Soto A, Solari L, Gotuzzo E, Acinelli R, Vargas D, Van der Stuyft P. Performance of an algorithm based on WHO recommendations for the diagnosis of smear-negative pulmonary TB in patients without HIV infection. Trop Med Int Health 2011;16:424-30

5. Mala G, Moser A, Dinant GJ, Spigt M. Why TB service providers do not follow treatment guideline in Ethiopia: a qualitative study. J Eval Clin Pract 2013.

6. Gallardo CR, Rigau D, Irfan A, Ferrer A, Cayla JA, Bonfill X, Alonso-Coello P. Quality of TB guidelines: urgent need for improvement. Int J Tuberc Lung Dis 2010;14:1045-51.

7. ISTC. International Standards for TB Care (ISTC) second edition. The Hague TB Coalition for Technical Assistance 2009.

8. Singh, S., Madge, S., Lipman, M. (2002) TB in primary care. Br J Gen Pract, 52(478),357-8.

9. Corless, J. A., Stockton, P. A., Myers, S. B., Davies, P. D. (2002) A world-wide internet survey of public knowledge about TB. Respir Med, 96(1),59-60.

10. Moro, M. L., Resi, D., Mezzetti, F., Borrini, B. M. (2003) [Diagnostic delay in patients with pulmonary TB]. Recenti Prog Med, 94(4),157-62.

11. Piot, M. (1967) A simulation model of case finding and treatment in TB control programmes. In, Geneva: WHO.

12. WHO. Global TB Report 2013 [accessed 15 Feb 2014].

13. Greenaway C, Menzies D, Fanning A, Grewal R, Yuan L, FitzGerald JM; Canadian Collaborative Group in nosocomial Transmission of TB. Delays in diagnosis among hospitalized patients with active TB: predictors and outcomes. Am J Respir Crit Care Med 2002; 165:927-93.

14. Lee CH, Lee MC, Lin HH, Shu CC, Wang JY, Lee LN, Chao KM. Pulmonary TB and delay in anti-tuberculous treatment are important risk factors for chronic obstructive pulmonary disease. PLosOne 2012; 7(5):e37978.

15. Uplekar M, Juvekar S, Morankar S, Rangan S, Nunn P. TB patients and practitioners in private clinics in India. Int J Tuberc Lung Dis 1998; 2:324-329.

16. Chung WS, Chang RE, Guo HR. Variations of care quality for infectious pulmonary TB in Taiwan: a population based cohort study. BMC Public Health 2007; 7:107

17. Hopewell PC, Pai M, Maher D, Uplekar M, Raviglione MC. International standards for TB care. Lancet Infect Dis 2006; 6:710-725.

18. Global TB WHO Report 2014

19. First Ethiopian national population based TB prevalence survey, Addis Ababa, July 2011, page 2

20. Int J Tuberc Lung Dis. 2014 Jun; 18(6):635-9. doi: 10.5588/ijtld.13.0417

21. HEAL-TB/KAPTLD. Strategy to mainstream gender in TB care and control in Ethiopia. J.M. Chakaya et al

22. R. McNerney, P. Daley, towards a point-of-care test for active tuber-culosis: obstacles and opportunities, Nat. Rev. Microbiol. 9 (2011)204-213. [17] M.D.

23. Perkins, P.M. Small, Partnering for better microbial diagnostics, Nat. Biotechnol. 24 (2006) 919-921.

24. E. Keeler, Reducing the global burden of TB: the contribution of improved diagnostics, Nature 49-57 (2006) e9848.

25. R. Mutetwa, et al., Diagnostic accuracy of commercial urinary lipoara-binomannan detection in African TB suspects and patients, Int. J. Tuberc. Lung Dis. 13 (2009) 1253-1259.

26. C. Boehme, et al., Detection of mycobacterial lipoarabinomannan with an antigen-capture ELISA in unprocessed urine of Tanzanian patients with suspected TB, Trans. R. Soc. Trop. Med. Hyg. 99 (2005)893-900.

27. K. Dheda, et al., Clinical utility of a commercial LAM-ELISA assay for TB diagnosis in HIV-infected

28. McLaughlin TJ, Soumerai SB, Willison D, et al. Adherence to national guidelines for drug treatment of suspected acute myocardial infarction: evidence for undertreatment in women and the elderly. Arch Intern Med. 1996; 156:799-805. 
29. European Secondary Prevention Study Group. Translation of clinical trials into practice: a European population-based study of the use of thrombolysis for acute myocardial infarction. Lancet. 1996; 347:1203-7.

30. Antman EM, Lau J, Kupelnick B. A comparison of results of meta-analyses of randomized control trials and recommendations of clinical experts: treatments for myocardial infarction. JAMA. 1992; 268:240-8.

31. Cabana MD, Rand CS, Powe NR, et al. Why don't physicians follow clinical practice guidelines? A framework for improvement. JAMA. 1999; 282:1458-65.

32. Hopewell PC, Pai M. TB, vulnerability, and access to quality care. JAMA. 2005;293:2790 - 3 .

33. Olle-Goig JE, Cullity JE, Vargas R. A survey of prescribing patterns for TB treatment amongst doctors in a Bolivian city. Int J Tuberc Lung Dis. 1999;3:74 - 8.

34. Prasad R, Nautiyal RG, Mukherji PK, Jain A, Singh K, Ahuja RC. Diagnostic evaluation of pulmonary TB: what do doctors of modern medicine do in India? Int J Tuberc Lung Dis. 2003; 7:52 - 7.

35. Shah SK, Sadiq H, Khalil M, Noor A, Rasheed G, Shah SM, et al. Do private doctors follow national guidelines for managing pulmonary TB in Pakistan? East Mediterr Health J. 2003;9:776 - 88.

36. Singla N, Sharma PP, Singla R, Jain RC. Survey of knowledge, attitudes and practices for TB among general practitioners in Delhi, India. Int J Tuberc Lung Dis. 1998;2:384 - 9.

37. Uplekar M, Pathania V, Raviglione M. Private practitioners and public health: weak links in TB control. Lancet. 2001;358:912-6.

38. Storla DG, Yimer S, Bjune GA. A systematic review of delay in the diagnosis and treatment of TB. BMC Public Health. 2008;8:15.

39. Godfrey-Faussett P, Kaunda H, Kamanga J, van Beers S, van Cleeff M, Kumwenda-Phiri R, et al. Why do patients with a cough delay seeking care at Lusaka urban health centres? A health systems research approach. Int J Tuberc Lung Dis. 2002;6:796-805.

40. Mauch V, Woods N, Kirubi B, Kipruto H, Sitienei J, Klinkenberg E. Assessing access barriers to TB care with the tool to estimate patients ' costs: pilot results from two districts in Kenya. BMC Public Health. 2011;11:43.

41. Gele AA, Sagbakken M, Abebe F, Bjune GA. Barriers to TB care: a qualitative study among Somali pastoralists in Ethiopia. BMC Res Notes. 2010;3:86.

42. Buregyeya E, Kulane A, Colebunders R, Wajja A, Kiguli J, Mayanja H, et al. TB knowledge, attitudes and health-seeking behaviour in rural Uganda. Int J Tuberc Lung Dis. 2011;15:938 - 42.

43. Hu A, Loo E, Winch PJ, Surkan PJ. Filipino women ' s TB care seeking experience in an urban poor setting: a socioecological perspective. Health Care Women Int. 2012;33:29-44.

44. Needham DM, Bowman D, Foster SD, Godfrey-Faussett P. Patient care seeking barriers and TB programme reform: a qualitative study. Health policy (Amsterdam, Netherlands). 2004;67:93 - 106.

45. Aye R, Wyss K, Abdualimova H, Saidaliev S. Illness costs to households are a key barrier to access diagnostic and treatment services for TB in Tajikistan. BMC Res Notes. 2010;3:340.

46. Wei X, Chen J, Chen P, Newell JN, Li H, Sun C, et al. Barriers to TB care for rural-to-urban migrant TB patients in Shanghai: a qualitative study. Trop Med Int Health. 2009;14:754 - 60.

47. Dimitrova B, Balabanova D, Atun R, Drobniewski F, Levicheva V, Coker R. Health service providers ' perceptions of barriers to TB care in Russia. Health Policy Plan. 2006;21:265 - 74.

48. Long $Q$, Li Y, Wang Y, Yue Y, Tang C, Tang S, et al. Barriers to accessing TB diagnosis for rural-to-urban migrants with chronic cough in Chongqing, China: a mixed methods study. BMC Health Serv Res. 2008;8:202.

49. Hane F, Thiam S, Fall AS, Vidal L, Diop AH, Ndir M, et al. Identifying barriers to effective TB control in Senegal: an anthropological approach. Int J Tuberc Lung Dis. 2007;11:539 - 43.

50. Madhukar Paia, Jessica Miniona, Karen Steingartb and Andrew Ramsayc. New and improved TB diagnostics: evidence, policy, practice, and impact. Wolters Kluwer Health | Lippincott Williams \& Wilkins. 2010;1070-5287

51. Bossuyt PM, McCaffery K. Additional patient outcomes and pathways in evaluations of testing. Med Decis Making 2009; 29:E30-E38. 


\section{Chapter 1}

52. Lord SJ, Irwig L, and Bossuyt PM. Using the principles of randomized controlled trial design to guide test evaluation. Med Decis Making 2009; 29:E1-E12.

53. WHO. Engaging Professional Association in TB Control. Report of the Meeting of the DOTS Expansion Working Group. WHO/HTM/TB/2009.413. WHO, Geneva, 2009.

54. Golub JE, Mohan $\mathrm{Cl}$, Comstock GW et al. Active case finding of TB: historical perspective and future prospects. Int. J. Tuberc. Lung Dis. 2005; 9: 1183-203.

55. Steyerberg EW. Clinical Prediction Models: A Practical Approach to Development, Validation, and Updating. New York: Springer; 2009

56. Wasson JH, Sox HC, Neff RK, Goldman L. Clinical prediction rules. Applications and methodological standards. N Engl J Med. 1985; 313:793-9. [PMID: 3897864]

57. Hayward RA, Kent DM, Vijan S, Hofer TP. Multivariable risk prediction can greatly enhance the statistical power of clinical trial subgroup analysis. BMC Med Res Methodol. 2006; 6:18. [PMID: 16613605]

58. Kattan MW, Vickers AJ. Incorporating predictions of individual patient risk in clinical trials. Urol Oncol. 2004; 22:348-52. [PMID: 15283895]

59. Biomarkers working group (2001). Biomarkers and surrogate endpoints: preferred definitions and conceptual framework. Clinical Pharmacology and Therapeutics, 69(3):89-95.

60. Phillips $\mathrm{M}$ et al. (2007). Volatile biomarkers of pulmonary TB in the breath. TB (Edinburgh, Scotland), $87(1): 44-5$

61. Kell DB (2006). Systems biology, metabolic modelling and metabolomics in drug discovery and development. Drug discoveryToday, 11(23-24):1085-1092.

62. Proctor PH. Endothelium-Derived Relaxing Factor and Minoxidil: Active Mechanisms in Hair Growth.Arch Dermatol.1989; 125(8):1146. oi:10.1001/archderm.1989.01670200122026

63. Chan ED, Chan J, Schluger NW (2001) What is the role of nitric oxide in murine and human host defense against TB?Current knowledge. Am J Respir Cell Mol Biol 25: 606-612.

64. Scanga CA, Mohan VP, Tanaka K, Alland D, Flynn JL, et al. (2001) The inducible nitric oxide synthase locus confers protection against aerogenic challenge of both clinical and laboratory strains of Mycobacterium TB in mice. Infect Immun 69: 7711-7717.

65. Liu PT, Modlin RL (2008) Human macrophage host defense against Mycobacterium TB. Curr Opin Immunol 20: 371-376.

66. Nicholson S, Bonecini-Almeida Mda G, Lapa e Silva JR, Nathan C, Xie QW, et al. (1996) Inducible nitric oxide synthase in pulmonary alveolar macrophages from patients with TB. J Exp Med 183: 2293-2302.

67. Rich EA, Torres M, Sada E, Finegan CK, Hamilton BD, et al. (1997) Mycobacterium TB (MTB)-stimulated production of nitric oxide by human alveolar macrophages and relationship of nitric oxide production to growth inhibition of MTB. Tuber Lung Dis 78: 247-255.

68. Rockett KA, Brookes R, Udalova I, Vidal V, Hill AV, et al. (1998) 1,25Dihydroxyvitamin D3 induces nitric oxide synthase and suppresses growth of Mycobacterium TB in a human macrophage-like cell line. Infect Immun 66: 5314-5321.

69. Schon T, Elmberger G, Negesse Y, Pando RH, Sundqvist T, et al. (2004) Local production of nitric oxide in patients with TB. Int J Tuberc Lung Dis 8: 1134-1137.

70. Choi HS, Rai PR, Chu HW, Cool C, Chan ED (2002) Analysis of nitric oxide synthase and nitrotyrosine expression in human pulmonary TB. Am J Respir Crit Care Med 166: 178-186.

71. Zumla A, Wallis R, Doherty M, Klein N, Parida S, Olesen O, Lång H, Vahedi M and Onyebujoh P. Joint TDR/EC expert consultation on biomarkers in TB: report of the joint TDR/EC expert consultation to evaluate the potential roles of biomarkers in the management of HIV-infected and HIV-uninfected patients with TB, Geneva, Switzerland, 2-3 July 2008

72. Diel R et al. (2008). Predictive value of a whole blood IFN-gamma assay for the development of active TB disease after recent infection with Mycobacterium TB. American Journal of Respiratory and Critical Care Medicine, 177(10):1164-1170.

73. Doherty TM et al. (2002). Immune responses to the Mycobacterium TB-specific antigen ESAT-6 signal subclinical infection among contacts of TB patients. Journal of Clinical Microbiology, 40(2):704-706. 
Chapter

\section{Quality of diagnosis and monitoring of tuberculosis in Northern Ethiopia: medical records-based retrospective study}




\section{ABSTRACT}

Objectives: To determine quality of diagnosis and monitoring of treatment response of patients with smear-negative pulmonary tuberculosis (TB) compared with smearpositive cases in Ethiopia.

Methods: A retrospective analysis of medical records of newly diagnosed pulmonary TB cases that were registered for taking anti-TB medication and had completed treatment between 2010 and 2012. We evaluated the percentage of cases that were managed according to the International Standards of Tuberculosis Care (ISTC) and compared smear-negative with smear-positive cases.

Results: We analyzed 1168 cases of which 742 (64\%) were sputum smear-negative cases. Chest radiography examination at diagnosis and microbiological testing at the end of the intensive phase of treatment was performed in a smaller proportion than in smearpositive TB cases (70\% vs. $79 \%$, P value $<0.001)$ and (70\% vs. 95\%, P value $<0.001)$, respectively.

Conclusions: Clinical actions recommended in the ISTC are of greatest importance in minimizing pitfalls in care of smear-negative TB yet were performed less often in smearnegative than smear-positive TB cases. 


\section{INTRODUCTION}

Despite available treatment that could cure $90 \%$ of cases, there were 8.7 million tuberculosis (TB) cases and 1.4 million deaths from TB worldwide in 2011 [1]. Moreover, of the 22 high-burden TB countries, only 14 reached the $85 \%$ target for treatment success among all new cases in 2010 [1].

The focus for TB control has moved towards reaching successful treatment of all cases of TB through offering a standard of care consistent with TB guidelines [2]. To achieve this, the ISTC provides a widely accepted protocol of care that all providers should follow in managing patients who have suspected TB.

The degree to which clinical actions specific for smear-negative TB are performed is largely unknown, owing to its relatively low public health priority [3] and there is an assumption that the prognosis for smear-negative cases is better than for smearpositive cases $[4,5]$. We hypothesized those clinical measures related to care for smearnegative TB patients may be poorly followed compared to smear-positive TB cases.

To understand the current diagnosis and monitoring of treatment response of TB, this study determined the proportion of TB cases in which clinical measures were followed according to ISTC 5 and 10 [2], in both smear-negative and smear-positive cases, diagnosed and treated in four different hospitals. A critical analysis of current clinical actions against these agreed standards, could lead to the identification of the most prevalent gaps in TB care. Accordingly, if measures are taken to bridge these adherence gaps in performed clinical actions, usual care will become more effective, for both smear-negative and smear-positive TB.

\section{METHODS}

\section{Study design}

We conducted a retrospective review of medical records of four hospitals in Ethiopia. All adults aged 18 years and above, newly diagnosed pulmonary TB cases that were registered for taking anti-TB medication and had completed treatment from September 2010 through December 2012, were included in the study. Patients were excluded if they: (1) had a previous history of TB; (2) had extra-pulmonary TB; (3) had no clinical data about treatment completion; (4) were documented to have a concurrent HIV infection, as there are differences in necessary clinical actions for these patients; and (5) if what was recorded in the patient's chart, TB treatment logbook and laboratory logbook was contradictory. All patients diagnosed with TB were also routinely tested for HIV. Currently the prevalence of HIV in Ethiopia is estimated to be $2.1 \%$ and $41 \%$ of TB patients are estimated to have co-infection with HIV $[6,7]$. 


\section{ISTC 5 on diagnosis}

Sputum smear microscopy is critical in the diagnosis of TB. Suspected patients are treated for smear-positive TB if the test is positive on admission. If the test is negative, but there is a strong suspicion of TB based on clinical symptoms, diagnosis of TB may be made by other means. According to ISTC 5, the following actions should be performed after a patient has an initial smear-negative test: (1) repeat the sputum test; (2) start antibiotic treatment; and (3) perform a Chest $\mathrm{x}$-ray radiography (CXR). Thereafter, patients suspected of having TB (e.g. compatible radiograph and clinical symptoms) should be treated as smear-negative TB patients. Patients who are not treated for TB should receive abroad based antibiotic to treat other possible bacterial infections. The antibiotic course should be completed.

\section{ISTC 10 on monitoring treatment response}

In addition to TB diagnosis, sputum smear microscopy has further use in monitoring treatment response. In both smear-negative and smear-positive, the treatment response should be monitored at the time of completion of the initial phase of treatment (2 months). If the sputum smear is positive at this stage, sputum smears should be examined again at 3 months, and if still positive, culture and drug susceptibility testing should be performed. However, the costs and complexities of routine cultures for TB as well as the delay in receiving results limit the widespread use of sputum culture. New diagnostic strategies using the more sensitive polymerase chain reaction (PCR) amplification of Mycobacterium tuberculosis deoxyribonucleic acid (DNA) have recently been introduced, but these may be too expensive for wide-spread use in resourcelimited settings $[8,9]$. Patients whose sputum smear microscopy was negative before treatment as well as after 2 months need no further sputum monitoring and should be monitored clinically, for which body weight is a useful progress indicator, because this should increase with successful treatment.

\section{Study setting}

Patients were recruited from four of the six hospitals in the Tigray region in Ethiopia. In these hospitals the Ethiopian TB guidelines are used for diagnosis and monitoring of treatment response. The ISTC recommendations are cross-referenced into the Ethiopian guidelines [10]. However, the former differ from the latter in that they recommend which clinical measures should be followed, whereas the Ethiopian guidelines describe the sequence in which the measures should be accomplished.

TB treatment consists of two phases, the intensive and continuation phases. During the first, ambulatory patients who live in communities far from the TB clinic are supplied with drugs and then referred to a health facility near their place of residence for 
Directly Observed Therapy under Supervision (DOTS) by a TB service provider on a daily basis. Patients living close to the TB clinic go to the hospital where their TB was diagnosed for DOTS every morning. TB patients are only admitted to hospital if they are very ill and are discharged when they can walk by them-selves; there is no isolation for those with positive sputum smears.

In the continuation phase, all patients are seen at the TB clinic every month on an ambulatory basis, irrespective of where DOTS was administered during the intensive phase. Monitored treatment response, all medications given, the bacteriologic response, and adverse reactions, are all recorded. Treatment is provided free of charge to all TB patients in Ethiopia.

\section{TB data records and data collection}

The register is maintained at local treatment units and comprises a list of all persons who have been diagnosed with TB. It contains entries for demographic and clinical characteristics including status of patients follow-up, laboratory tests results and treatment outcome. Occasionally the healthcare worker in charge of a TB unit does evaluate practices recommended by national guidelines and the ISTC, and reminds local staff to document these practices on the clinical encounter form. These clinical actions are cross-checked to evaluate completeness, accuracy and promptness of record-keeping, and for the purpose of programme auditing.

Table1. Patient profile at diagnosis

\begin{tabular}{lcccccc}
\hline \multicolumn{5}{c}{ Proportion of variables by hospital settings } \\
\cline { 2 - 6 } & Hospital 1 & Hospital 2 & Hospital 3 & Hospital 4 & Total & P-value \\
Baseline Characteristics & $n=306$ & $n=279$ & $n=283$ & $n=300$ & $n=1168$ & \\
\hline Age (years; mean (SD)) & $38.8(15.9)$ & $39.2(17.1)$ & $35.4(14.1)$ & $36.1(15.3)$ & $37.4(15.7)$ & 0.017 \\
Male gender (n (\%)) & $170(56 \%)$ & $156(56 \%)$ & $153(54 \%)$ & $165(55 \%)$ & $644(55 \%)$ & 0.981 \\
Mean weight (kg (SD)) & $45.8(8.0)$ & $45.9(7.5)$ & $44.7(9.1)$ & $46.7(8.8)$ & $45.8(8.4)$ & 0.077 \\
Smear-negative TB (n (\%)) & $234(77 \%)$ & $169(61 \%)$ & $171(60 \%)$ & $168(56 \%)$ & $742(64 \%)$ & $<.001$ \\
Smear-positive TB (n (\%)) & $72(23 \%)$ & $110(39 \%)$ & $112(40 \%)$ & $132(44 \%)$ & $426(36 \%)$ & $<.001$ \\
Symptoms compatible & & & & & \\
with TB (n (\%)) & & & & & $<.001$ \\
Cough & $178(58 \%)$ & $279(100 \%)$ & $236(83 \%)$ & $273(91 \%)$ & $966(83 \%)$ & $<.001$ \\
Haemoptysis & $5(2 \%)$ & $11(4 \%)$ & $64(23 \%)$ & $67(22 \%)$ & $147(13 \%)$ & $<.001$ \\
Night Sweating & $260(85 \%)$ & $276(99 \%)$ & $163(58 \%)$ & $206(69 \%)$ & $905(78 \%)$ & $<.001$ \\
Auxiliary Temperature & $242(79 \%)$ & $13(5 \%)$ & $50(18 \%)$ & $118(39 \%)$ & $423(36 \%)$ & $<$ \\
$>37.8^{\circ} \mathrm{C}$ & & & & & \\
\hline
\end{tabular}

SD standard deviation 
A structured data collection checklist was designed and tested before data collection. A team of four registered nurses working in TB clinics attended a 1-day workshop about the structured data sheet provided by the principal investigator, and were trained for data collection. Data were extracted from TB clinic registries based on paper patient charts, paper TB treatment logbooks and paper laboratory logbooks. From patient charts we collected demographic characteristics and clinical features (symptoms, CXR findings and prescription of broad-spectrum antibiotics). Laboratory results (smear results, type of TB) and clinical monitoring of body weight were collected from TB treatment logbooks. We counter-checked TB treatment logbooks and laboratory logbooks to confirm if the sputum smear test was performed.

\section{Data analysis}

Data were analyzed using SPSS version 19 (SPSS, Inc., Chicago, IL, USA). Descriptive analyses were employed to calculate the proportion of TB cases that were diagnosed and monitored according to ISTC protocol. Differences between smear-negative and smear-positive cases were analyzed by applying Pearson's Chi-square tests for dichotomous outcomes and Mann-Whitney $U$ tests for continuous outcomes. For testing differences between the four different hospitals, we used Pearson's Chi-square and KruskalWallis tests.

Personal identifiers were not collected and data were analysed anonymously. The study protocol was reviewed and approved by the Institutional Review Board of the Research and community service of College of Health Sciences of Mekelle University (ERC 0304/2010). A letter of permission to review medical records was obtained from the office of the Director of Ayder Referral Hospital.

\section{RESULTS}

\section{Patient profile at diagnosis}

We selected 1168 medical records of newly diagnosed pulmonary TB cases that were registered for taking anti-TB medication and had completed treatment from September 2010 to September 2012 (Table 1). In total, 742 (64\%) were smear-negative and 426 (36\%) smear-positive. Patients' age at diagnosis was in the range of 18-86 years with a mean of 37 years and 644 (55\%) were male. Almost all patients' weights were measured at diagnosis with a remarkably low mean of $45.8 \mathrm{~kg}$. The most common recorded symptoms compatible with TB were cough (83\%), night sweating (78\%), fever (36\%) and hemoptysis (13\%), but there were significant differences between the different hospitals. 


\section{Performed clinical actions}

Microbiological testing by sputum smear microscopy was always performed (100\%) at diagnosis and at the end of the continuation phase of treatment (Table 2). However, microbiological testing at the end of the intensive phase of treatment, to monitor response to treatment, was performed in a higher proportion in smear-positive than smear-negative cases $(96 \%$ vs. $70 \%$, P value $<0.001)$. A significant difference ( $\mathrm{P}$ val$\mathrm{ue}<0.001$ ) in the proportion of patients who received CXR examination at diagnosis was observed between the hospitals. Interestingly, CXR examination was performed at diagnosis less often in smear-negative than in smear-positive cases (70\% vs. $79 \%, \mathrm{P}$ value $<0.001$ ). In Hospital 1, only half of smear-negative cases were evaluated by CXR examination compared to $100 \%$ in smear-positive TB cases (P value $<0.001$ ). Broad-spectrum antibiotics were never prescribed. Weight was always measured at diagnosis in all hospitals. However, weight monitoring at the end of the intensive was averagely performed less often in smear-negative than smear-positive TB cases $(27 \%$ vs. $52 \%$, P value $<0.001)$. However, monitoring of weight at the end of the continuation phase was rarely done across all settings except in Hospital 4.

\section{DISCUSSION}

Our findings show that a higher proportion of clinical actions were omitted during diagnosis and monitoring of treatment response in smear-negative compared with smearpositive TB cases, despite the fact that the clinical actions were especially important for the former. We also observed large differences between hospitals regarding ISTC adherence.

Performing CXR examination at diagnosis in smear-negative TB cases was only performed in $70 \%$ of cases, and significantly less often in smear-negative than in smearpositive cases. This is a determining factor especially in the diagnosis of smear-negative TB where diagnostic evaluation is challenging, even when all appropriate investigations have been conducted [11]. Besides, clinical monitoring of body weight at the end of the intensive phase, which is an important indicator of treatment response in smearnegative cases, was performed less often than in smear-positive TB cases. Hence, it is difficult to understand how smear-negative TB patients were monitored clinically.

Microbiological testing at the end of the intensive phase was on average done less in smear-negative than smear-positive TB cases. It is also difficult to understand how patients whose sputum smear microscopy was negative at the start of treatment, were rechecked at the end of the intensive phase either to rule out non-response to treatment or an error at the time of initial diagnosis (i.e. a true smear-positive patient was misdiagnosed as smear-negative). It is surprising that there was little use of such easily measured and inexpensive markers such as sputum and weight monitoring. 
The overall performed clinical measures also differed significantly between the hospitals despite the available basic infrastructure to provide TB services being of comparable quality. Significant differences in performance of CXR examination at diagnosis, microbiological testing at the end of intensive phase and monitoring of body weight were observed between hospitals. For example, Hospital 4 almost fully met the desired standard in diagnosis and monitoring treatment response in all TB cases, while Hospital 3 performed poorly. Although we did not evaluate infrastructural differences between hospitals, a study in a similar resource poor settings reported no relationship between quality of structural facilities and quality of TB care in hospitals [12]. However, healthcare providers' expertise in management of TB was linked to quality of care [13]. Therefore, dissimilarities in performed clinical actions in our study are related to findings by WHO and others [14-16] that have shown that clinicians often deviate from internationally recommended TB management. Strategies that increased clinicians' adherence to set standards in some hospitals need to be identified and introduced to the less adherent hospitals in order to improve the quality of TB care.

\section{Relevance for healthcare}

The performance of the WHO's diagnostic algorithm is far from good in real life [17], since it is often negatively influenced by local factors and practice-related context $[14,18]$. Evaluating quality of TB care may be more realistic in routine care, if the overall performed clinical actions are assessed instead of assessing the sequence of actions in the diagnostic algorithm [19]. In our study, we assessed the overall performed clinical actions in the targeted standards which are of greatest need in minimizing pitfalls in diagnosis and monitoring response of treatment in smear-negative TB patients and reported that they were done less often in smear-negative than smear-positive TB cases. Our findings add that care offered to smear-negative cases continues to be deviant from the standards, compared to smear-positive TB cases. This could be due to the assumption that the prognosis for smear-negative cases is better than for smearpositive cases. However, this assumption can only be valid if microscopy procedures are followed completely and reliably [20-22]. It has regrettably been shown that in many low-income settings, laboratory procedures are not optimal [14, 23, and 24]. Therefore, there is a need to explore the reasons behind poor standardized clinical work-up in smear-negative compared to smear-positive TB cases. 


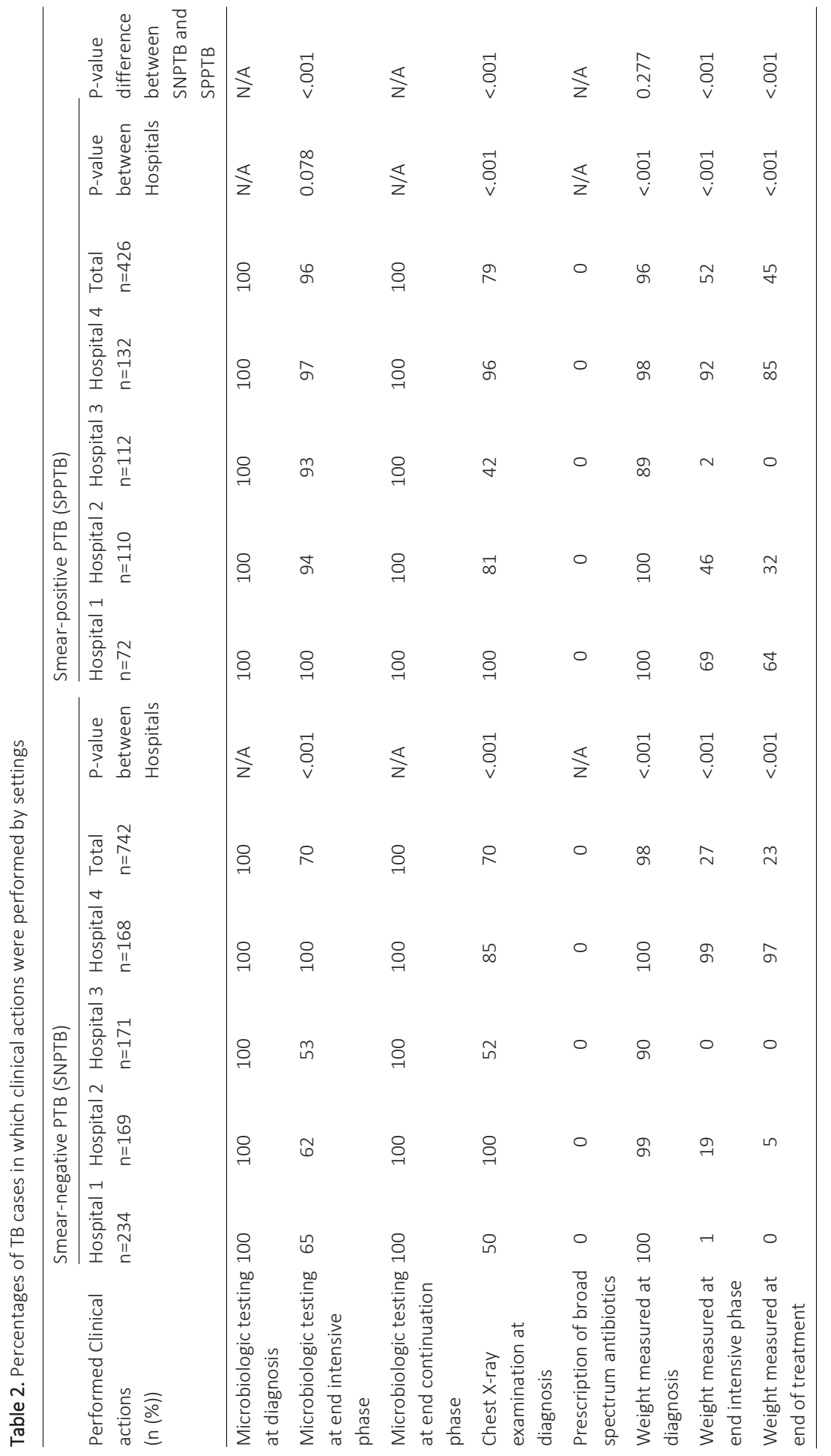




\section{Strengths and limitations}

Our study primarily reviewed clinical measures recommended by ISTC which represent activities that TB care providers perform mostly themselves and are consistent with the national Ethiopian TB guideline. The main limitation of our study was that absence of data did not always signify the absence of services. It is possible that, services may have been provided to patient (e.g. prescription of broad spectrum antibiotics), but not documented. Since incomplete records were taken as non-adherence, adherence could have been larger in reality. Besides, we were limited in the number of clinical measures we could evaluate because our analysis relied on TB cases with complete medical records. However our findings from analysis of the best cases possible, with complete medical records, echo a fundamental principle of TB control espoused by Piot more than 40 years ago, that a substantial proportion of TB cases occur among individuals who fail to access high quality diagnostic and treatment services [25].

\section{CONCLUSIONS}

Performing clinical measures recommended by ISTC is of the greatest importance in optimizing care for smear-negative TB. Recommended actions were unfortunately performed less often in smear-negative than smear-positive TB cases. We perceived this to be a habitual bias by TB healthcare providers, who apparently still consider managing smear-positive cases to be the most effective strategy to curb the TB epidemic. This treatment adherence gap would be significantly closed if strategies that increase compliance to ISTC are identified in hospitals that best meet the desired standards. There is also a need to explore qualitatively the reasons and hidden assumptions behind poor standardized clinical work-up in smear-negative as compared to smear-positive TB cases. 


\section{REFERENCES}

1. World Health Organization. Global Tuberculosis Report. Geneva: WHO, 2012.

2. International Standards for Tuberculosis Care. International Standards for Tuberculosis Care (ISTC), 2nd edn. The Hague: Tuberculosis Coalition for Technical Assistance, 2009.

3. World Health Organization. Treatment of Tuberculosis Guidelines, 4th edn. Geneva: WHO, 2010.

4. Tiemersma EW, van der Werf MJ, Borgdorff MW, Williams BG and Nagelkerke NJ. Natural history of tuberculosis: duration and fatality of untreated pulmonary tuberculosis in HIV negative patients: a systematic review. PLoS One 2011; 6: e17601.

5. Shah NS, Cavanaugh JS, Pratt R, Cain KP, Wells C, Laserson K, et al. Epidemiology of smear-negative pulmonary tuberculosis in the United States, 1993-2008. Int J Tuberc Lung Dis 2012; 16: 1234-1240.

6. Berhane Y. The AIDS pandemic is still expanding its horizon: comprehensive, harmonized and large scale responses needed. Ethiop J Health Dev 2004; 18: 67-70.

7. Federal Ministry of Health. Tuberculosis, TB/HIV and Leprosy Prevention and Control Strategic Plan, 2007/8 - 2009/10. Addis Ababa: Federal Ministry of Health, 2007.

8. Lawn SD, Bekker LG, Middelkoop K, Myer L and Wood R. Impact of HIV infection on the epidemiology of tuberculosis in a peri-urban community in South Africa: the need for age-specific interventions. Clin Infect Dis 2006; 42: 1040-1047.

9. Scott LE, McCarthy K, Gous N, Nduna M, Van Rie A, Sanne I, et al. Comparison of Xpert MTB/RIF with other nucleic acid technologies for diagnosing pulmonary tuberculosis in a high HIV prevalence setting: a prospective study. PLoS Med 2011; 8: e1001061.

10. Federal Ministry of Health. Guidelines for Clinical and Programmatic Management of TB, Leprosy and TB/HIV in Ethiopia. Addis Ababa: Federal Minsitry of Health, 2012.

11. Ashkin D, Hollender ES and Narita M. "Won't get fooled again" (by tuberculosis). Chest 1999; 116: 856857.

12. Hongoro C, McPake B and Vickerman P. Measuring the quality of hospital tuberculosis services: a prospective study in four Zimbabwe hospitals. Int J Qual Health Care 2005; 17: 287-292.

13. Chung WS, Chang RE and Guo HR. Variations of care quality for infectious pulmonary tuberculosis in Taiwan: a population based cohort study. BMC Public Health 2007; 7: 107.

14. Mala G, Moser A, Dinant GJ and Spigt M. Why tuberculosis service providers do not follow treatment guide-line in Ethiopia: a qualitative study. J Eval Clin Pract 2014; 20: 88-93.

15. Uplekar M, Juvekar S, Morankar S, Rangan S and Nunn P. Tuberculosis patients and practitioners in private clinics in India. Int J Tuberc Lung Dis 1998; 2: 324-329.

16. World Health Organization. Involving private practitioners in tuberculosis control issues; interventions, and emerging policy framework. Geneva: WHO, 2001.

17. Soto A, Solari L, Gotuzzo E, Acinelli R, Vargas D and Van der Stuyft P. Performance of an algorithm based on WHO recommendations for the diagnosis of smear-negative pulmonary tuberculosis in patients without HIV infection. Trop Med Int Health 2011; 16: 424-430

18. Gallardo CR, Rigau D, Irfan A, Ferrer A, Cayla JA, Bonfill X, et al. Quality of tuberculosis guidelines: urgent need for improvement. Int J Tuberc Lung Dis 2010; 14: 1045-1051.

19. Ahmad RA, Matthys F, Dwihardiani B, Rintiswati N, de Vlas SJ, Mahendradhata Y, et al. Diagnostic workup and loss of tuberculosis suspects in Jogjakarta, Indonesia. BMC Public Health 2012; 12: 132.

20. Aber VR, Allen BW, Mitchison DA, Ayuma P, Edwards EA and Keyes AB. Quality control in tuberculosis bacteriology. 1. Laboratory studies on isolated positive cul-tures and the efficiency of direct smear examination. Tubercle 1980; 61: 123-133.

21. Siddiqi K, Lambert ML and Walley J. Clinical diagnosisof smear-negative pulmonary tuberculosis in lowincomecountries: the current evidence. Lancet Infect Dis 2003; 3: 288-296.

22. Gilpin C, Kim SJ, Lumb R, Rieder HL and Van Deun A Working Group on Sputum Smear Microscopy. Critical appraisal of current recommendations and practices for tuberculosis sputum smear microscopy. Int J Tuberc Lung Dis 2007; 11: 946-952. 


\section{Chapter 2}

23. Khan MS, Dar O, Sismanidis C, Shah K and Godfrey-Faussett P. Improvement of tuberculosis case detection and reduction of discrepancies between men and women by simple sputum-submission instructions: a pragmatic randomized controlled trial. Lancet 2007; 369: 1955-1960.

24. Prasad R, Nautiyal RG, Mukherji PK, Jain A, Singh K and Ahuja RC. Treatment of new pulmonary tuberculosis patients: what do allopathic doctors do in India? Int J Tuberc Lung Dis 2002; 6: 895-902.

25. Piot M. A simulation model of case finding and treatment in tuberculosis 
Chapter

\section{Why tuberculosis service providers do not follow treatment guideline in Ethiopia: a qualitative study}




\section{ABSTRACT}

Aim: Little is known about the reasons why tuberculosis (TB) service providers do not follow TB treatment guidelines. A better understanding of their real-world experiences associated with non-compliance with guideline may highlight opportunities for enhanced TB care. Whether the problem is actual or perceived may also affect the type of intervention needed to overcome the barrier. This study aimed to explore TB service providers' reasons for not complying with TB treatment guidelines.

Method: A descriptive qualitative design was used. Focus group discussions were held with 39 service providers involved in TB care. The study was carried out in the Tigray region of Ethiopia, in four of the six zonal hospitals. Data were analyzed using content analysis.

Results: The main reasons for non-compliance with TB treatment guidelines were: (1)insufficient diagnostic modalities, limiting the capacity to correctly diagnose TB and provide the right regimen; (2) ambiguity in guideline recommendations especially for specific subgroups of patients, making it difficult to prioritize them to the right regimen; and (3) poor documentation concerning referred patients, with no appropriate examination and difficulty obtaining information from previously treated patients.

Conclusion: Non-compliance may reflect valid questions about the applicability of guideline recommendations in the local context of health care. Special consideration should be given to previously treated patients, as an important factor influencing guideline compliance. Guideline compliance may also be improved by a better strategy to retest those patients whose initial tests are negative in health care contexts where TB is frequently missed. 


\section{INTRODUCTION}

The primary aims of tuberculosis (TB) control programmes are early diagnosis and prompt treatment of infectious cases in order to limit transmission [1]. The World Health Organization (WHO) has developed specific outcome measures to evaluate TB control programmes. To achieve the intended outcome, the WHO has recommended diagnosis and treatment guidelines for the implementation of national tuberculosis programmes for each country $[2,3]$. Treatment outcomes are recorded internationally, and targets of $70 \%$ case detection and $85 \%$ cure in smear-positive pulmonary TB have been set [4]. However, these broad outcome measures do not provide detailed insight into the pathways of clinical care, nor do they identify reasons for missing the targets.

Recent studies have reported multiple pitfalls in the diagnosis and treatment of TB patients, even in regions where TB might be considered a rarity [5-7]. Clinicians and patients have identified suboptimal diagnostic processes [8], uncertainty about appropriate clinical practice in the treatment of specific subgroups of patients, lack of integration and coordination of health services [9], as well as excessively prolonged prescription of anti-TB drugs [10]. This despite the availability of highly effective TB therapy and an intensive worldwide effort to improve access to the diagnostic and treatment interventions embodied in the directly observed treatment-short course (DOTS) strategy. This pitfalls echoes a fundamental principle of TB control espoused by Piot more than 40 years ago, that a substantial proportion of TB cases occur among individuals who fail to access high-quality diagnostic and treatment services [11].

Little information is available to explain why TB service providers do not follow TB guidelines in regions where TB prevalence is high. Studies to document quality deficits in TB care in these regions have taken the form of surveys using closed questions [12, 13]. In addition, surveys of barriers have relied on the providers' opinions and may not accurately reflect how problematic a barrier actually is, and the surveys may not have captured all the relevant barriers. Furthermore, studies that attempted to explore physician compliance with guidelines have focused on general practitioners' knowledge and physicians' attitude, rather than practice-related and context-specific reasons for not following guidelines $[12,13]$. In this study, we explored reasons given by TB service providers for their non-compliance with TB guidelines. The objective was to explore health care providers' practice-related and context-specific reasons for not following the guidelines, since a better understanding of their real-world experiences associated with non-compliance with guidelines may reveal opportunities for enhanced TB care. We also examined whether a barrier is actual or perceived, as this may also affect the type of intervention needed to overcome it. 
Chapter 3

\section{METHODS}

\section{Study design}

This study used a descriptive qualitative design to explore TB service providers' practicerelated and context-specific reasons for not complying with TB treatment guidelines. Semi-structured interviews were held with 39 service providers involved in TB care. Data were analyzed using content analysis.

\section{Study setting}

The study was carried out in the Tigray region of Ethiopia, in four of the six zonal hospitals, ranging in size from 36 to 500 beds. In these hospitals, TB is diagnosed and registered, and anti-TB treatment is started. The Ethiopia TB guideline is used for the diagnosis, treatment and monitoring of treatment response in adult patients who have, or are suspected of having, TB. Sputum smear microscopy is the main critical element in the diagnosis of active cases of TB. TB treatment consists of two phases, the intensive and continuation phases. During the intensive phase of treatment, patients who live in communities far away from the TB clinic are supplied with drugs and then referred to a health facility near their place of residence for DOTS by a TB service provider on a daily basis. Patients living close to the TB clinic go to the hospital where their TB was diagnosed for DOTS every morning. In the continuation phase of treatment, all patients are seen at the TB clinic every month on an ambulatory basis, irrespective of where DOTS was administered during the intensive phase. Response to treatment is monitored and recorded, including all medications given, bacteriologic response and adverse reactions. Treatment is provided free of charge to all TB patients in Ethiopia.

\section{TB treatment guideline}

Suspected TB patients with positive sputum smear are treated for TB. While suspected TB patients with negative sputum smears are managed according to a diagnostic algorithm that includes treatment with broad spectrum antibiotic and chest $X$-ray examination to determine whether or not the patient has smear-negative TB [3, 14]. Patients having chest $\mathrm{X}$-ray findings compatible with TB are treated for smear-negative TB. Other suspected patients not treated for TB receive a broad-spectrum antibiotic to treat bacterial infection. Response to antibiotics is assessed within 3-5 days into treatment, and, if there is no improvement, anti-TB treatment is initiated.

Patients are prescribed anti-TB regimens based on their previous TB treatment history: as new patients and previously treated patients. New patients are treated with 'new patient regimen', while previously treated patients receive a retreatment regimen containing first-line drugs $[3,14]$. All patients treated for TB are evaluated for treatment 
response. TB treatment is considered failed if the specimen obtained at the end of the third month is still smear-positive; hence sputum culture and drug susceptibility testing (DST) should be performed. TB patients whose treatment has failed or other patient groups with high likelihood of multidrug-resistant TB (MDR-TB) should be started on an empirical MDR regimen. TB patients returning after defaulting or relapsing from their first treatment course may receive the retreatment regimen containing first-line drugs if country-specific data show low or medium levels of MDR in these patients or if such data are unavailable. Furthermore, in settings where DST results are not yet routinely available to guide the management of these individual patients, the empirical regimens will continue throughout the course of treatment.

\section{Participants}

A total of 39 TB service providers participated. They were six health officers, 12 nurses, six laboratory technicians, five internists and 10 general practitioners. Included were participants who were either involved in TB guideline implementation or provided services to TB patients in government-run hospitals. Participants were directly involved in the diagnosis and treatment of TB patients, with the exception of the laboratory technicians. Excluded were health care professionals who were not involved in providing care to TB patients. In order to get a broad view of the topic under study, we selected a range of professionals with different perspectives, using purposive sampling [15]. We made sure that the sample included males and females, young/inexperienced and older/experienced professionals and specialists working in the hospitals, to foster discussion among participants.

\section{Ethical considerations}

Ethical approval for the study was obtained from Tigray Bureau of Health, College of Health Sciences, Mekelle University and four health institutions. The aim of the study was explained to the participants, who gave informed written consent.

\section{Data collection}

Data collection took place between June and August 2011. A total of six focus group discussions (FGDs) were held, each with six to eight people participating. The FGDs took place at a location that was accessible to all participants. All FGDs were led by an experienced moderator with an understanding of the topic under study, who was a trained health worker and a native speaker of the local/official language of the study area. A questioning route for the FDGs [16] was prepared, with open-ended questions, probes and prompts. The questions were based on information from the current literature (sensitizing concepts) [17], and had been reviewed by stakeholders and adapted accord- 
ingly. The topics were quality deficits, compliance with TB guidelines and information transfer. The topics in the questioning route were presented one by one, allowing sufficient discussion of each topic. Since data collection and analysis happened simultaneously, the questions were adjusted according to the findings of previous data collection rounds, and more questions were added or modified as we proceeded through the research process to obtain more in-depth information about the topic. For example, we added questions about adherence to DOTS principles. Data were collected in three rounds, with two FGDs conducted in each round. Each FGD lasted 2 hours. The FGDs were tape recorded and field notes were written down in a notebook by an assistant moderator. Data were transcribed verbatim in Amharic language and translated into English.

\section{Data analysis}

Data analysis used the qualitative content analysis approach based on the constant comparison method [17]. Codes and categories emerged inductively from the data through careful examination by the researcher. First, the transcripts from the FGDs and the field notes were read to get a general impression, after which the data were coded using open coding. The open codes were compared and contrasted and categories were defined. These categories were again compared and contrasted, and correct categories and subcategories emerged. This was done within and across the data sets. The ongoing data analysis took place throughout the study. After the first two FGDs with professionals, the research team read the translations and discussed emerging categories and subcategories, and decided which issues needed further elaboration. This procedure was followed in three analytical sessions, one after each round of FGDs [15]. We reached data saturation after four FGDs and used the remaining two FGDs to confirm the findings.

\section{Trustworthiness}

To ensure the reliability of the study data, we used investigator and methodological triangulation [18]. Data triangulation was secured by including different professional groups (health officers, nurses, laboratory workers, etc.). Investigator triangulation was applied by involving two different investigators in the data collection and analysis process. Methodological triangulation was ensured by gathering different types of data, such as FGD transcripts and field notes. Furthermore, to improve the transferability of the findings to different settings and contexts, a detailed description was prepared of the setting, sampling, sample size, inclusion and exclusion criteria, interview procedure and topics. This should help readers decide whether the findings of this study are transferable to their own context [18]. 


\section{RESULTS}

Five categories of factors influencing TB service providers' compliance with the guidelines emerged. They were listed in order of the most to the least important reason for non-compliance with guidelines as insufficient diagnostic modalities, ambiguity in guideline recommendations, poor documentation, health service situation and professional social context, respectively.

\section{Insufficient diagnostic modalities}

The TB service providers reported that the national TB programme guideline recommendations were insufficiently helpful in situations where there was a lack of diagnostic modalities. They believed that the major cause of non-adherence to the guidelines was having sputum smear microscopy as the only critical modality for the diagnosis of active TB cases.

We have very deficient diagnostic modalities; we cannot do sputum culture or blood culture (. . . ) the diagnostic guidelines are not adaptable to the situation on the ground.

Respondents also reported that they doubted the results of sputum smear microscopy tests. In their practice, they observed that the sputum smear microscopy test gave false-negative or false-positive results even in patients with clear clinical symptoms. Consequently, TB service providers had adopted a practice of requesting both sputum smear microscopy test and chest X-ray examination simultaneously at the beginning of the diagnostic process. This is, however, not in accordance with the diagnostic algorithm, and results in overreliance on chest X-ray radiography for diagnosis.

To diagnose pulmonary TB we can do only sputum smear microscopy test, which usually gives negative results in our set-up (. . .) If those pulmonary TB suspects that might be positive are told that they are negative, this is the worst thing for the transmission of TB. For this reason, we do order both the sputum smear microscopy and chest X-ray together because the sputum microscopy test usually gives us a negative result.

\section{Ambiguity in guideline recommendations}

TB service providers experienced ambiguity in prioritizing previously treated patients who had relapsed, failed or defaulted treatment. Prioritization is important to provide the right regimen for these patients as recommended in the guidelines. Guidelines recommend that patients should be categorized for retreatment according to their likelihood of developing drug resistance and the availability of alternative diagnostic 
modalities. Respondents reported that they could only prioritize these patients for retreatment based on the sputum smear microscopy examination, which was of poor quality. Thus, their clinical judgment was not supported by these recommendations in everyday practice.

We do not have faith in the sputum smear microscopy test, and if there is no culture service, I should be allowed to use my clinical judgment not only in smearnegative patients, but also in categorizing previously treated patients, but the guide-line does not allow this. I mean the guideline should support what we have.

Respondents also claimed that guidelines were not sufficiently relevant for specific subgroups of patients, particularly patients who developed drug-induced toxicity or patients with special requirements such as those with TB meningitis. A source of particular concern was the difficulty of choosing the appropriate treatment regimen if the preferred combination of anti-TB drugs is unavailable. The preferred presentation of most anti-TB drugs was lacking because they were donated to hospitals as fixed drug combinations (FDCs). Consequently, respondents reported that guideline recommendations did not reflect the best practice in their situation, where only FDCs were available in unsuitable combinations.

Drugs may not be available even in fixed doses preparations; let alone the single drugs. Then we are blamed for not sticking to the guideline recommendations and not sticking to the right regimens, but they don't supply the drugs. We don't have any other option, so how should we treat these patients? What we do have currently is Rifampicin, Isoniazid, Pyrazinamide and Ethambutanol (RHZE) instead of $\mathrm{RHZ}$ and we are still treating the patient with RHZE and streptomycin like a retreatment case. This is a retreatment regimen. 'What should I do'? Tell me what to do when the right presentation of FDCs is unavailable? How can I remove the Ethambutanol from the RHZE?

\section{Poor documentation}

Poor referral linkage was a theme repeatedly reported by respondents in all FGDs. The TB service providers did not have the necessary information about which tasks had been completed, and they also lacked information on why certain interventions had already been applied to the patients referred to them. Respondents reported that patients were referred without a systematic clinical history, examination and appropriate investigation. Hence, they experienced difficulties in the care of such patients due to a loss of transparency in rational clinical decision-making.

Most of the time patients come to you without proper referral papers or with no clear evidence why the patient was started on anti-TB drugs and the evidence is 
difficult to trace back. First of all, we don't know how the patient was diagnosed with TB and we rely on the patients to tell us whether their sputum was negative or positive, but most patients wouldn't know. This is what I usually see as a deficit in the quality of TB care.

The TB service providers also reported key information being lacking from patient records. They expressed their frustration about the TB management of previously treated patients, where they knew little about their clinical history. In most cases, these patients can hardly provide any accurate past and present treatment information. Many respondents were unable to trace the information about diagnosis and medication previously taken by patients.

We usually face these difficulties with the care of patients who have been treated with anti-TB drugs previously. We don't know what type of TB they've been having and what kind of medication they've been taking, because these patients don't know anything about their disease condition. We face a dilemma on how to manage the patient because the whole history is missing, and this is common in many big hospitals in the country, not only in ours.

\section{Health service situation}

The TB service providers observed that almost all patients were faced with limited access to health service, due to geographical or economic disadvantages. In addition, patients were obliged to make multiple visits to TB clinics often over a considerable period of time. Such patients warrant making exceptions to some components of ideal care. The respondents were concerned that without exceptions to guideline recommendations, such patients might be at significantly higher risk of missing care due to loss to follow-up.

Most patients come from far away to the TB clinic, and are poor peasant farmers. So we cannot strictly follow the guideline recommendations (especially the diagnostic algorithm and DOTS principle) because patients will be lost to followup and may either go to another place or may not come at all next time.

Respondents expressed negative expectations about DOTS principles. In particular, they considered DOTS to be ineffective in the prevention of drug resistance. As a result, antiTB drugs were distributed at the bedside to inpatients without direct observation, while outpatients were provided with the anti-TB drugs for weeks without any professional supervision.

Do we really believe DOTS works to prevent the development of MDR-TB, which is actually emerging and is rising alarmingly? I think that the DOTS principles are 
a difficult ideology to practice but that there's currently no other option than DOTS to prevent the development of drug-resistant TB.

\section{Professional social context}

Respondents mentioned that medical professionals in government-run and privately run hospitals had little interest in following national guideline recommendations, especially where they perceived them to conflict with their own interests.

In private practice there is a tendency not to stick to the diagnostic algorithm. If I tell a patient you have bronchitis or something else, which is not TB, and I give the patient only amoxicillin and then advise them to come back after a month or after two weeks for re-evaluation, then it's not uncommon to see the same patient with an anti-TB drug the next day, in one of the private clinics. Even in government-run hospital settings this follow-up may not be perfect, because I may not be there and then the patient is kind of lost in between, this is my concern. In a rural healthcare setting it may be easier to do this, where I'm the only one who follows the same patient and can eventually follow the guidelines. But the issue here is, if I follow the guidelines and give the patient antibiotics; other healthcare providers may start anti-TB when I am no longer involved with the same patient.

In addition, junior health care providers reported that influential colleagues deviated from the guidelines based on their expert knowledge or individual treatment preferences.

With regard to the categorization of pediatric patients in particular, most of them are categorized as category three because of a lack of diagnostic modalities and the influence of seniors.

\section{DISCUSSION}

\section{Summary of main findings}

The aim of this study was to explore Ethiopian TB service providers' practice-related and context-specific reasons for not complying with TB treatment guidelines. Major reasons for non-compliance were insufficient diagnostic modalities, ambiguity in guideline recommendations, poor documentation, health service situation and professional social context.

The TB service providers in this study had developed their own routine and style of diagnostic evaluation. They had adopted a practice of requesting both a sputum smear 
microscopy test and a chest X-ray examination at the beginning of the diagnostic process. This finding is similar to that of a study in India, where poor quality of the sputum smear microscopy test was also considered to be the reason for non-compliance with diagnostic guidelines [19]. These authors also observed a tendency to over-rely on chest X-ray for the diagnosis of pulmonary TB. A specific aspect in our setting was that respondents had developed negative expectations about the results of sputum smear microscopy tests. This contextual factor, which is unrelated to the actual performance of this diagnostic tool, substantially influenced their compliance with the guidelines.

Several barriers hampering the diagnosis and treatment of specific subgroups of patients have been described previously, especially in a regions where TB is considered a rarity [7]. In that study, the most common reasons for non-compliance involved issues of older patients and immigrant populations. TB was often considered difficult to ascertain in elderly patients, and the authors feared drug-induced toxicity in these patients. The most critical problem in immigrant patients was the lack of information on drug resistance patterns [7]. Consequently, clinical decision making about the initial treatment regimen for immigrant patients was perceived as a great challenge [6].

In our study, the most problematic subgroup was that of previously treated patients who had relapsed or failed or defaulted on treatment. It was difficult to prioritize these patients for the retreatment regimens available. Correctly diagnosing TB and providing the right regimen for these patients was a major problem, due to insufficient diagnostic modalities, lack of information on previous medical history and unavailability of information on drug resistance patterns. The consequences were numerous. Respondents observed diagnostic errors that exposed patients to inappropriate anti-TB drugs, leading to drug-induced toxicity.

The question arises if non-compliance is justified in these cases. Our findings suggest that non-compliance may reflect valid questions about the applicability of guideline recommendation to this specific subgroup of patients. Prescription of non-standard medication regimens has been reported in other studies [20, 21], and our findings confirm that this practice continues. The most commonly reported reasons for not using recommended regimens were doubts about the efficacy of the regimen for the recommended period, and the perceived risk of toxicity [20,21]. Our findings do not enable us to confirm these reasons, because the use of inappropriate regimens was associated with either a lack of separate medicines or unavailability of FDCs in the preferred presentation. Priority was given to average patients with no complications, and fewer investments were being made to improve the quality of TB care for patients that do not fall into the category of average patients. But as Vijan pointed out, 'Not all patients are the same. Treating them as such not only minimizes autonomy, but it is also a recipe for inefficiency' [22].

Most previous studies have acknowledged that unsupervised treatment was taking place under DOTS. DOTS was perceived to be useful only for a few average patients, suggesting a mismatch between patient needs and the existing strategy [23]. Geograph- 
ic and economic disadvantages were the most common reasons for non-compliance with DOTS in our study, as has also been found in other studies [24-26]. Surprisingly, unsupervised treatment was provided by our respondents because of negative expectations about a core principle of DOTS that is effective prevention of the development of drug resistance. The rise of MDR-TB in our setting [27] signals a failure to adhere to the DOTS strategy. Although randomized controlled trials of DOTS have shown no benefit in terms of adherence to medication [28-30], it is still believed to be the best strategy available for TB control [31].

\section{Strengths and limitations of our study}

The strengths of this study were that it included a range of different TB service providers from a number of different health care settings. This allowed us to identify divergent perceptions and experiences, assumptions and beliefs, and to explore what actually happens at the patients' bedside. It is also important to note that almost all the respondents claimed to have expert knowledge and were willing to comply with the guidelines, although we made no attempt to evaluate their knowledge of and attitude towards the guidelines. The purposive sampling may have led to the selection of respondents who were optimistic. Finally, the findings of this study should be interpreted against the background of its setting (with poorly developed infrastructure).

\section{Implication for future research and practice}

TB guidelines may not improve the quality of care when available diagnostic tests are, or are perceived to be, poor. Our findings indicate that the degree of trust that TB service providers had in the diagnostic test results had a great impact on compliance with the guidelines. Thus, addressing their negative perception of sputum smear microscopy may be just as important as scaling up the diagnostic test. Furthermore, there was dilemma among TB service providers about relying only on sputum microscopy in active case finding. The underlying conflict was a lack of alternative diagnostic modalities when the initial test result does not correspond with the patient's clinical presentation. A better strategy to retest those patients whose initial tests are negative in settings where TB is frequently missed may improve compliance with the guideline.

A recent review of the TB guidelines showed that the overall methodological quality of the guideline was low [32]. Several domains of the guidelines, such as applicability to specific local situations, contributed to their poor performance [32]. Our findings offer real-life examples of what is needed to customize TB guidelines to local contexts of health care in order to improve compliance. We recommend developing intervention strategies that are not tailored only to average patients. Consideration should be given to specific subgroups as an important influence on guideline compliance. 


\section{CONCLUSION}

Non-compliance may reflect valid questions about the applicability of guideline recommendations to the local context of health care. Special consideration should be given to previously treated patients, as an important influence on compliance with guidelines. Guideline compliance may also be improved by a better strategy to retest those patients whose initial tests are negative in regions where TB is frequently missed. 


\section{REFERENCES}

1. van der Werf, M. J. \& Borgdorff, M. W. (2007) Targets for tuberculosis control: how confident can we be about the data? Bulletin of the World Health Organization, 85 (5), 370-376.

2. WHO (2008) Global Tuberculosis Control: Surveillance, Planning, Financing. Geneva: World Health Organization.

3. WHO (2009) Treatment of Tuberculosis Guidelines. Geneva: World Health Organization.

4. Maher, D., Blanc, L. \& Raviglione, M. (2004) WHO policies for tuberculosis control. Lancet, 363 (9424), 1911.

5. Singh, S., Madge, S. \& Lipman, M. (2002) Tuberculosis in primary care. The British Journal of General Practice, 52 (478), 357-358.

6. Corless, J. A., Stockton, P. A., Myers, S. B. \& Davies, P. D. (2002) A world-wide internet survey of public knowledge about tuberculosis. Respiratory Medicine, 96 (1), 59-60.

7. Moro, M. L., Resi, D., Mezzetti, F. \& Borrini, B. M. (2003) Diagnostic delay in patients with pulmonary tuberculosis. Recenti Progressi in Medicina, 94 (4), 157-162.

8. Metcalf, E. P., Davies, J. C., Wood, F. \& Butler, C. C. (2007) Unwrapping the diagnosis of tuberculosis in primary care: a qualitative study. The British Journal of General Practice, 57 (535), 116-122.

9. Moro, M. L., Resi, D., Lelli, B., Nicoli, A., Gagliotti, C. \& Falcone, F. (2005) Barriers to effective tuberculosis control: a qualitative study. The International Journal of Tuberculosis and Lung Disease, 9 (12), 13551360 .

10. Valin, N., Hejblum, G., Borget, I., Mallet, H. P., Antoun, F., Che, D. \& Chouaid, C. (2010) Factors associated with excessively lengthy treat-ment of tuberculosis in the eastern Paris region of France in 2004. BMC Public Health, 10, 495.

11. Piot, M. (1967) A Simulation Model of Case Finding and Treat-ment in Tuberculosis Control Programmes. Geneva: World Health Organization.

12. Munro, S. A., Lewin, S. A., Smith, H. J., Engel, M. E., Fretheim, A. \& Volmink, J. (2007). Patient adherence to tuberculosis treatment: a systematic review of qualitative research. Plos Medicine, 4 (7), 1230- 1245

13. Noyes, J. \& Popay, J. (2007) Directly observed therapy and tuberculosis: how can a systematic review of qualitative research contribute to improving services? A qualitative meta-synthesis. Journal of Advanced Nursing, 57 (3), 227-243.

14. FMoH (2012) Guidelines for Clinical and Programmatic Management of TB, Leprosy and TB/HIV in ETHIOPIA, Addis Ababa.

15. Holloway, I. \& Wheeler, S. (2002) Qualitative Research in Nursing. Oxford: Blackwell.

16. Krueger, R. A. \& Casey, M. A. (2000) Focus Groups. A Practical Guide for Applied Research. Thousand Oaks, CA: Sage Publications.

17. Strauss, A. \& Corbin, J. (1998) Basics of Qualitative Research Techniques and Procedures for Developing Grounded Theory. London: Sage Publications.

18. Lincoln, Y. \& Guba, E. G. (1985) Naturalistic Inquiry. Newbury Park, CA: Sage Publications.

19. Prasad, R., Nautiyal, R. G., Mukherji, P. K., Jain, A., Singh, K. \& Ahuja, R. C. (2002) Treatment of new pulmonary tuberculosis patients: what do allopathic doctors do in India? The International Journal of Tuberculosis and Lung Disease, 6 (10), 895-902.

20. Chengsorn, N., Bloss, E., Anekvorapong, R., et al. (2009) Tuberculosis services and treatment outcomes in private and public health care facilities in Thailand, 2004-2006. The International Journal of Tuberculosis and Lung Disease, 13 (7), 888-894.

21. Quy, H. T., Lonnroth, K., Lan, N. T. \& Buu, T. N. (2003) Treatment results among tuberculosis patients treated by private lung specialists involved in a public-private mix project in Vietnam. The International Journal of Tuberculosis and Lung Disease, 7 (12), 1139-1146.

22. Vijan, S. (2000) Are we overvaluing performance measures? Effective Clinical Practice, 3 (5), 247-249. 
23. Atkins, S., Lewin, S., Ringsberg, K. C. \& Thorson, A. (2012) Towards an empowerment approach in tuberculosis treatment in Cape Town, South Africa: a qualitative analysis of programmatic change. GlobHealth Action, 5, 1-11.

24. O’Boyle, S. J., Power, J. J., Ibrahim, M. Y. \& Watson, J. P. (2002) Factors affecting patient compliance with anti-tuberculosis chemo-therapy using the directly observed treatment, short-course strategy (DOTS). The International Journal of Tuberculosis and Lung Disease, 6 (4), 307-312.

25. Jaramillo, E. (1999) Encompassing treatment with prevention: the path for a lasting control of tuberculosis. Social Science and Medicine, 49 (3), 393-404.

26. Lonnroth, K., Tran, T. U., Thuong, L. M., Quy, H. T. \& Diwan, V. (2001) Can I afford free treatment?: perceived consequences of health care provider choices among people with tuberculosis in Ho Chi Minh City, Vietnam. Social Science and Medicine, 52 (6), 935-948.

27. Integrated Regional Information Networks (IRIN) Africa (2010) Racing to Contain MDR-TB. IRIN: Ethiopia.

28. Zwarenstein, M., Schoeman, J. H., Vundule, C., Lombard, C. J. \& Tatley, M. (1998) Randomised controlled trial of self-supervised and directly observed treatment of tuberculosis. Lancet, 352 (9137), 1340- 1343.

29. Garner, P. (1998) What makes DOT work? Directly observed therapy. Lancet, 352 (9137), 1326-1327.

30. Newell, J. N., Baral, S. C., Pande, S. B., Bam, D. S. \& Malla, P. (2006) Family-member DOTS and community DOTS for tuberculosis control in Nepal: cluster-randomised controlled trial. Lancet, 367 (9514), 903909.

31. Davies, P. D. (2003) The role of DOTS in tuberculosis treatment and control. American journal of respiratory medicine, 2 (3), 203-209.

32. Gallardo, C. R., Rigau, D., Irfan, A., Ferrer, A., Cayla, J. A., Bonfill, X. \& Alonso-Coello, P. (2010) Quality of tuberculosis guidelines: urgent need for improvement. The International Journal of Tubercu-losis and Lung Disease, 14 (8), 1045-1051. 

Chapter

\section{Predicting tuberculosis in clinical suspects and among smear negative cases, with routinely available diagnostic tools in Ethiopia}




\section{ABSTRACT}

Objectives: To evaluate the performance of predictors for estimating the probability of tuberculosis (TB) (1) among all clinical suspects and (2) among smear negative cases.

Methods: From September to November 2013, a total of 426 consecutive patients, admitted to two hospitals in Tigray Ethiopia, with suspicion of TB, were evaluated by sputum smear microscopy and chest radiography in accordance with the Ethiopian guidelines of the National Tuberculosis and Leprosy Program. The performance of predictors for estimating the probability of TB in clinical suspects and smear negative TB cases were based on the results of culture tests.

Results: Among all cases the strongest predictive variables both in univariate and multivariate analysis of culture-positive TB, were a positive smear test (OR 172; Cl 23.231273.54) and having chest radiography lesions compatible with TB (OR 10.401; Cl;5. 862-18.454). However, among smear negative cases almost all variables showed no significant difference between culture-positive and culture-negative TB. The regression model had a good predictive performance, for identifying culture-positive TB among patients (AUC 0.84) with clinical suspicion, but it was rather poor in patients with a negative smear result (AUC 0.64). Combining all the predictors in the model compared to only the independent significant variables did not really improve its performance to identify culture-positive (AUC 0.84 to 0.87 ) and culture-negative (AUC 0.64 to 0.69) TB.

Conclusions: Our findings suggest that neither the hallmark symptoms nor radiographic findings are invariably associated with culture-negative compared to culture-positive TB, among smear negative suspects. Therefore, predictive models based on clinical variables will not be useful to discriminate patients with culture negative from culturepositive TB among smears negative cases. 


\section{INTRODUCTION}

Pulmonary tuberculosis (TB) with culture-negative, but positive clinical and chest radiographic findings is often encountered in routine practice in low-income countries. These cases are mostly also smear-negatives and their diagnoses have been problematic, which has been a matter of concern in recent decades [1, 2]. The clinical form of presentation is insidious and early diagnosis is often difficult if TB is not advanced [3]. Without a standardized clinical work up, the rate of diagnostic error has been estimated as high as 35\% to 52\% [4-6]. However, in settings with adequate resources, allowing for comprehensive diagnostic work-up and the exclusion of other respiratory diseases, a substantial proportion of the cases is diagnosed as culture-negative TB [7, 8]. Despite the considerable incidence of culture-negative TB, little is known about its clinical manifestations compared to culture-positive TB among smear negative cases.

Recognizing and diagnosing culture-negative TB is clinically challenging. The Nguyen et al. and a Hong Kong based study in the early 1980s [9, 10], found a lower frequency of cavitation in culture-negative pulmonary TB compared with culture-positive subjects. These findings indicate that, culture-negative pulmonary TB is an early disease state with low mycobacterial burden. Nguyen et al. and others [9, 11-13] observed a reduced frequency of clinical symptoms and radiographic lesions in cases with culture-negative TB, but their comparison groups (culture-positive) had both smear negative and smear positive cases. Inclusion of smear positive cases may potentially lead to different findings compared with a situation where the analysis is restricted only to smear negative TB. Therefore, we hypothesize that culture-negative TB cases may present with no significant differences in clinical and radiographic abnormalities when compared to those with culture-positive among smear-negative cases.

In addition, while reviewing deaths due to respiratory infections in Africa, several authors [14-19] found that missed TB diagnoses were likely due to less symptomatic states of TB premortem. Therefore, if bacteriological confirmation is needed before initiating the anti-TB treatment as per the Ethiopian TB guideline [20], it will result in far too many culture-negative TB cases left untreated, as there is no gold standard test for the diagnosis [7]. Moreover, the clinical definitions in existing guidelines are rather vague [21-23] and do not allow to classify patients according to their probability of TB.

While application of predictive modelling in patients with clinical suspicion of TB has been described before, these studies usually combined only independent significant predictive variables into the decision models [24-28]. In addition, information from a single predictor is often insufficient to provide reliable estimates of diagnostic probabilities or risks [29-30]. In particular for pragmatic reasons, clinicians in Ethiopia use all available information for each patient to determine whether TB is indeed present or absent. Therefore, in this study, we evaluated the performance of predictors for estimating the probability of TB when all versus only significant variables are considered; (1) among all clinical suspects and (2) among smear negative cases. 


\section{METHODS}

\section{Study setting, population and data collection}

This study was conducted at the Aydar and Mekelle Hospitals, 500 and 350 inpatient beds public sector referral hospitals respectively, that serve approximately 18 million people in Northern Ethiopia. From September to November 2013, a total of 426 consecutive patients, admitted to these hospitals, with suspicion of TB, were evaluated in accordance with the Ethiopian guidelines of the National Tuberculosis and Leprosy Program [17]. The study included all patients aged $\geq 18$ years, with a history of cough of $>3$ weeks, night sweats, fever for 1 month, weight loss and/or loss of appetite. All suspects were asked to produce two sputum samples (spot and early morning sample), used for culture testing. Patients unable to produce sputum and unwilling to give written informed consent were excluded from the study. Patients were also excluded from the study if they had discontinued anti-TB therapy in the past 3 months. Furthermore, we collected data in four main domains 1) patient history, 2) physical examination, 3) chest radiograph, and 4) sputum smear results. Personal identifiers were not collected and data were analyzed anonymously. The study protocol was reviewed and approved by the Institutional Review Board of the Research and community service of College of Health Sciences of Mekelle University (ERC 0304/2010).

Diagnosis of culture-positive TB was based on Mycobacterium tuberculosis growth in at least one of the first three sputum cultures. Smear-negative TB was defined as no acid fast bacillus (AFB) identified in the initial three sputum smears, while smearpositive TB was defined as at least one positive AFB smear. Culture-negative TB was defined as clinical and/or radiographic presentation consistent with TB, three initial mycobacterial sputum cultures negative, and no evidence of other respiratory disease.

Only early morning sputum specimens submitted to the hospital TB laboratory as part of routine sputum smear microscopy test was used for culture examinations. Sputum specimens were decontaminated on arrival at the reference laboratory using $2 \%$ sodium hydroxide and $0.5 \% \mathrm{~N}$-acetyl-cysteine for $25 \mathrm{~min}$, then neutralized to $\mathrm{pH} 7$, concentrated by centrifugation (3000xg for $15 \mathrm{~min}$ ) and inoculated into a single Mycobacterium Growth Indicator (MGIT) Tube 960 (MGIT, Becton Dickinson Microbiology Systems, Sparks, MD, USA) [32]. Smears were made from isolates obtained from the MGIT tubes, stained by the Ziehl Neelsen staining method, and examined under $100 \mathrm{x}$ magnifications using a light microscope for the presence of AFB. The growth on AFB positive MGIT tubes were further inoculated into two Lowenstein-Jensen slants, one containing sodium pyruvate. The cultures were examined twice a week and their rate of growth and colonial morphologies recorded.

The chest radiography lesions were categorized in terms of the involved lung field and the pattern of involvement. The involved field was categorized as upper and lower lung fields; left and right side of lung affected with lesions. The radiographic pattern was 
categorized as normal, or abnormal with lesions consistent with TB [31]. "Normal" pattern was defined as the absence of any abnormal lesion on chest radiography. A targeted physical examination was performed for height, weight, axillary or oral temperature, pulse rate, respiration rate and blood pressure. Chest radiography was read by local site investigators.

\section{Statistical analysis}

Univariate comparisons were performed using the $\chi 2$ test and Fisher's exact test for categorical variables and Student's t-test for continuous variables where appropriate. In this study, active TB was determined by a culture-positive result. Receiver operating characteristic (ROC) analysis was used to compare the accuracy of each predictive variable compared to culture results. The area under the ROC curve (AUC) was used as a measure of diagnostic accuracy. Under common practice, AUCs of $0.60-0.69,0.70-$ $0.79,0.80-0.89$ and $0.90-1.0$ were considered to correspond respectively to 'poor', 'fair', 'good' and 'excellent' diagnostic accuracy.

Unconditional logistic regression models were used to discriminate the outcome of culture results in clinical suspects and among smear negative cases and generate odds ratios (OR) and 95\% confidence intervals $(\mathrm{Cl})$ as estimates of effect size. Independent variables were utilized in logistic regression models in two ways: continuous and binary. Binary values were determined by median values. Best cutoff values were chosen for those continuous variables with values that discriminate culture-negative from culturepositive TB among smear-negative TB cases using ROC. For both continuous and binary independent variables, following initial models that included all variables, a second model that excluded nonstatistically significant variables $(P>0.05)$ was run. The HosmerLemeshow test was used to assess the fit of the logistic regression model. Estimates of sensitivity, specificity, and AUC were determined by final model fit. Standard errors for the AUC were calculated using the Mann-Whitney method. All statistical analyses were performed using SAS ver. 9.2 (SAS Institute, Cary, NC, USA). Statistical significance was at two-tailed $\mathrm{P}<0.05$.

\section{RESULTS}

Among the 426 enrolled cases, 255 (60\%) had an abnormal chest radiograph, of which 192 (75\%) had lesions compatible with TB (Figure 1). 46 (24\%) patients with chest radiography compatible with TB had positive results on sputum smear microscopy test and were therefore diagnosed as smear positive TB. Of these, 43 (98\%) were confirmed culture-positive cases. The remaining 146 (76\%) patients had negative sputum smears, while having a positive chest radiography and were therefore considered to have smear negative TB. However, 78 (64\%) of these patients were culture-negative TB cases. None 
of the patients with normal CXR had a positive smear test. A total of 72 patients without culture results were excluded from final analysis ( 2 with positive smears and 33 with negative smears among patients with abnormal CXR and 37 with negative smears among patients with normal CXR).

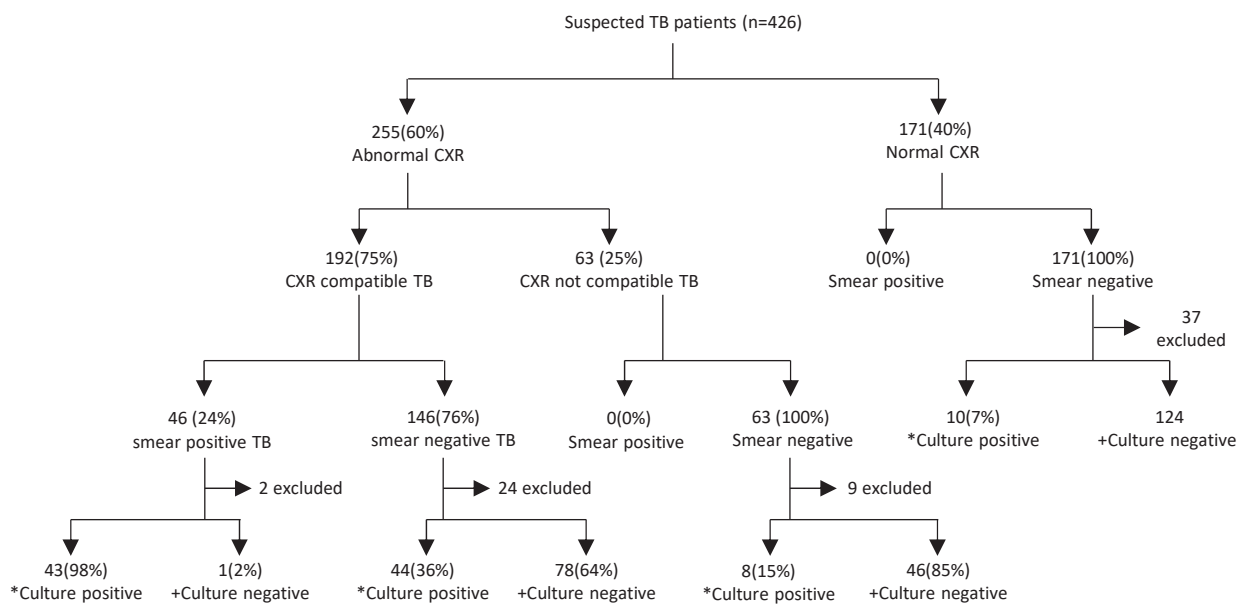

Figure 1. Participant flow diagram

*Total culture positive cases ( $n=105)$; +Total culture negative cases ( $n=249)$; Total excluded cases (because culture test not done) $(n=72)$

Predictive variables of culture-positive TB cases among patients with clinical suspicion are given in Table 1 . The results of the univariate analysis revealed that patients previously treated with anti-TB drugs (OR 2.72; Cl: 1.57-4.69), were more likely to have culture-confirmed TB than never treated patients. In addition, upper lung fields (OR 4.76; $\mathrm{Cl}$ : 2.52-8.99) and having bilateral lesions (OR 5.58; $\mathrm{Cl}$ : 2.85-10.91) seemed to be related to culture-positive TB. However, lesions present in the lower lung fields and on either side of the lungs, was less significantly related. The strongest predictive variables both in univariate and multivariate analysis of culture-positive TB, were a positive smear test (OR 172; $\mathrm{Cl}$ 23.23-1273.54) and having chest radiography lesions compatible with TB (OR 10.40; Cl5.86-18.45). A prediction model based on only the independent significant predictors (sputum smear microscopy and chest radiography) had a good performance by ROC analysis (AUC 0.84) for diagnosing culture-positive TB (Figure 2). Combining all predictors in the model compared to only the independent significant variables did not really improve its performance to identify culture-positive TB (AUC 0.84 to 0.87). The discriminating ability of the model neither showed much difference to rightly classify culture positive TB (AUC rose from 82 to 85 ). 
Table 1: Predictive risk factors of culture-positive compared to culture-negative TB among all clinical suspects with culture results $(n=354)$.

\begin{tabular}{|c|c|c|c|c|c|c|}
\hline \multirow[b]{2}{*}{$\begin{array}{l}\text { Predictive } \\
\text { variables }\end{array}$} & \multirow[b]{2}{*}{$\begin{array}{l}\text { Culture-positive } \\
\text { for TB }(n=105)\end{array}$} & \multirow[b]{2}{*}{$\begin{array}{l}\text { Culture- } \\
\text { negative for TB } \\
(n=249)\end{array}$} & \multirow[b]{2}{*}{$\begin{array}{l}\text { Univariate Odds Ratio } \\
(95 \% \mathrm{Cl})\end{array}$} & \multicolumn{3}{|l|}{ Model 1} \\
\hline & & & & $\beta(\mathrm{SE})$ & $\begin{array}{l}\text { Multivariate Odds Ratio } \\
(95 \% \mathrm{Cl})\end{array}$ & P Value \\
\hline \multicolumn{7}{|l|}{ Sex } \\
\hline Female & $40(25 \%)$ & $119(75 \%)$ & $0.67(0.42-1.07)$ & $-0.489(0.35)$ & $0.613(0.31-1.23)$ & 0.167 \\
\hline Male & $65(33 \%)$ & $130(67 \%)$ & 1.0 & & 1.0 & \\
\hline Age & & & & & & 0.423 \\
\hline $45+$ & $38(27 \%)$ & 104(73\%) & $0.89(0.47-1.67)$ & $-0.007(0.557)$ & $0.993(0.33-2.96)$ & 0.990 \\
\hline $25-44$ & $46(33 \%)$ & $94(67 \%)$ & $1.19(0.64-2.21)$ & $0.449(0.55)$ & $1.567(0.54-4.59)$ & 0.413 \\
\hline $0-24$ & $21(29 \%)$ & $51(71 \%)$ & 1.0 & & 1.0 & \\
\hline BMI & & & & & & 0.605 \\
\hline $\mathrm{BMI}<16$ & $15(26 \%)$ & $42(74 \%)$ & $0.98(0.50-1.93)$ & $-0.451(0.47)$ & $0.637(0.26-1.59)$ & 0.335 \\
\hline BMI 16>18.50 & $43(35 \%)$ & $78(65 \%)$ & $1.51(0.92-2.50)$ & $-0.004(0.390)$ & $0.996(0.46-2.1)$ & 0.992 \\
\hline BMI 18.5+ & $47(27 \%)$ & $129(73 \%)$ & 1.0 & & 1.0 & \\
\hline \multicolumn{7}{|l|}{ History of anti-TB } \\
\hline Previously treated & d33(47\%) & $37(53 \%)$ & $2.72(1.57-4.69)$ & $0.495(0.39)$ & $1.641(0.77-3.52)$ & 0.203 \\
\hline Never treated & $65(25 \%)$ & 198(75\%) & 1.0 & & 1.0 & \\
\hline \multicolumn{3}{|l|}{ Duration of cough } & & & & 0.998 \\
\hline $\begin{array}{l}13 \text { weeks and } \\
\text { above }\end{array}$ & $25(36 \%)$ & $44(64 \%)$ & $1.50(0.83-2.69)$ & $0.025(0.44)$ & $1.03(0.43-2.43)$ & 0.955 \\
\hline 7-12weeks & $27(29 \%)$ & $65(71 \%)$ & $1.09(0.63-1.90)$ & $0.022(0.40)$ & $1.02(0.47-2.24)$ & 0.957 \\
\hline 1-6 weeks & $52(28 \%)$ & $137(72 \%)$ & 1.0 & & 1.0 & \\
\hline Lung fields & & & & & & 0.643 \\
\hline Lower fields & $20(36 \%)$ & $35(64 \%)$ & $1.97(1.05-3.68)$ & $0.411(0.471)$ & $1.509(0.60-3.80)$ & 0.382 \\
\hline Upper fields & $29(58 \%)$ & $21(42 \%)$ & $4.76(2.52-8.99)$ & $0.351(0.520)$ & $1.420(0.51-3.94)$ & 0.500 \\
\hline Unspecified & $56(23 \%)$ & 193(77\%) & 1.0 & & 1.0 & \\
\hline Lung sides & & & & & & 0.742 \\
\hline Left & 20(39\%) & $32(61 \%)$ & $2.84(1.46-5.54)$ & $0.168(0.54)$ & $1.182(0.41-3.41)$ & 0.757 \\
\hline Bilateral & $27(55 \%)$ & $22(45 \%)$ & $5.58(2.85-10.91)$ & $0.577(0.54)$ & $1.780(0.62-5.11)$ & 0.284 \\
\hline Right & $23(39 \%)$ & $36(61 \%)$ & $2.90(1.50-5.50)$ & $0.164(0.54)$ & $1.178(0.41-3.42)$ & 0.764 \\
\hline Unspecified & $35(18 \%)$ & $159(82 \%)$ & 1.0 & & 1.0 & \\
\hline \multicolumn{7}{|l|}{ Smears results } \\
\hline Positive smears & $43(98 \%)$ & $1(2 \%)$ & $172.00(23.23-1273.54)$ & $4.352(1.05)$ & $77,668(9.89-609.68)$ & 0.001 \\
\hline Negative smears & $62(20 \%)$ & $248(80 \%)$ & 1.0 & & 1.0 & \\
\hline \multicolumn{7}{|l|}{ CXR findings } \\
\hline $\begin{array}{l}\text { Compatible with } \\
\text { TB }\end{array}$ & $87(52 \%)$ & $79(48 \%)$ & $10.401(5.86-18.45)$ & $1.660(0.43)$ & $5.261(2,280-12,141)$ & 0.001 \\
\hline $\begin{array}{l}\text { Not Compatible } \\
\text { with TB }\end{array}$ & $18(10 \%)$ & $170(90 \%)$ & 1.0 & & 1.0 & \\
\hline
\end{tabular}

$B M I$ Body Mass Index, CXR Chest x-ray 


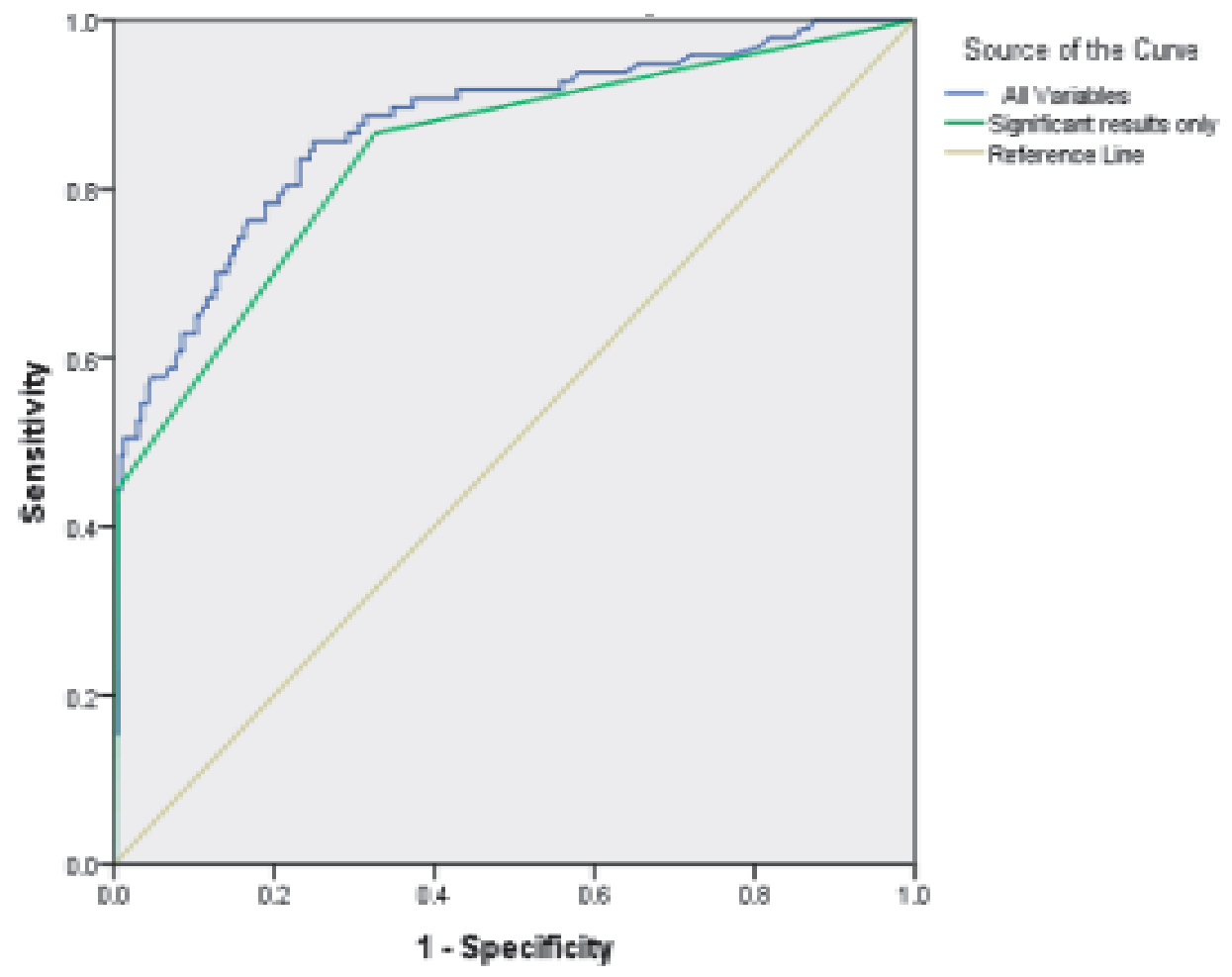

Figure 2. Receiver operator curve characteristics for the prediction of culture positive TB from culturenegative among clinical suspects $(n=354)$.

Almost all variables showed no significant difference between culture-positive and culture-negative TB among smear negative cases (Table 2). Female sex (OR 0.33; Cl: 0.150.71 ), was the only independent negative predictor of culture-positive TB in both analyses, and could be due to less severe lung lesions compared to males. The ROC curve for female sex as the only independent predictor compared to combinations of all predictors (Figure 3), both reported poor clinical performance of the model (AUC from 0.64 to 0.69$)$. 
Table 2: Predictive risk factors of culture-negative compared to culture-positive among smear negative TB cases $(n=146)$

\begin{tabular}{|c|c|c|c|c|c|c|}
\hline & & & & Model 1 & & \\
\hline Predictive variables & $\begin{array}{l}\text { Culture-negative } \\
\text { TB }(n=78)\end{array}$ & $\begin{array}{l}\text { Culture-positive } \\
\text { TB }(n=44)\end{array}$ & $\begin{array}{l}\text { Univariate Odds } \\
\text { Ratio } \\
(95 \% \mathrm{Cl})\end{array}$ & $\beta(S . E)$ & $\begin{array}{l}\text { Multivariate } \\
\text { Odds Ratio } \\
(95 \% \mathrm{Cl})\end{array}$ & P Value \\
\hline \multicolumn{7}{|l|}{ Sex } \\
\hline Female & $46(77 \%)$ & $14(23 \%)$ & $0.33(0.15-0 . .71)$ & $-1.13(0.40)$ & $0.325(0.15-0.71)$ & 0.005 \\
\hline Male & $32(52 \%)$ & $30(38 \%)$ & 1.0 & & & \\
\hline Age (group) & & & & & & 2.67 \\
\hline $45+$ & $43(67 \%)$ & $21(33 \% 0$ & $0.78(0.23-2.68)$ & $0.74(0.91)$ & $2.10(0.36-12.43)$ & 0.414 \\
\hline $25-44$ & $27(60 \%)$ & $18(40 \%)$ & $1.07(0.30-3.79)$ & $1.30(0.93)$ & $3.67(0.59-22.78)$ & 0.163 \\
\hline $0-24$ & $8(62 \%)$ & $5(38 \%)$ & 1.0 & & & \\
\hline Body Mass Index (BMI) & & & & & & 0.721 \\
\hline $\mathrm{BMI}<16$ & $23(74 \%)$ & $8(26 \%)$ & $0.55(0.21-1.45)$ & $-0.43(0.540)$ & $0.650(0.22-1.88)$ & 0.462 \\
\hline BMI 16>18.50 & $22(60 \%)$ & $15(40 \%)$ & $1.07(0.46-2.52)$ & $-0.05(0.52)$ & $0.952(0.35-2.61)$ & 0.924 \\
\hline BMI 18.5+ & $33(61 \%)$ & $21(39 \%)$ & 1.0 & & & \\
\hline \multicolumn{7}{|l|}{ History of anti-TB } \\
\hline Previously treated & $21(57 \%)$ & $16(43 \%)$ & $1.58(0.71-3.53)$ & $0.26(0.48)$ & $1.294(0.51-3.29)$ & 0.588 \\
\hline Never treated & $54(68 \%)$ & $26(32 \%)$ & 1.0 & & & \\
\hline Duration of cough & & & & & & 0.909 \\
\hline 13 weeks and above & $22(65 \% 0$ & $12(35 \%)$ & $0.92(0.37-2.27)$ & $0.22(0.53)$ & $1.249(0.44-3.53)$ & 0.674 \\
\hline 7-12weeks & $23(66 \%)$ & $12(34 \%)$ & $0.88(0.36-2.16)$ & $0.15(0.52)$ & $1.162(0.42-3.25)$ & 0.774 \\
\hline 1-6weeks & $32(63 \%)$ & $19(37 \%)$ & 1.0 & & & \\
\hline Lung fields with lesions & & & & & & 0.521 \\
\hline Lower fields & $22(65 \%)$ & $12(35 \%)$ & $1.02(0.42-2.44)$ & $0.47(0.56)$ & $1.605(0.53-4.84)$ & 0.401 \\
\hline Upper fields & $15(60 \%)$ & $10(40 \%)$ & $1.24(0.48-3.22)$ & $0.64(0.60)$ & $1.899(0.58-6.19)$ & 0.288 \\
\hline Unspecified & $41(65 \%)$ & $22(35 \%)$ & 1.0 & & & \\
\hline Lung sides with lesions & & & & & & 0.693 \\
\hline Left & $20(67 \%)$ & $10(33 \%)$ & $0.91(0.32-2.62)$ & $-0.65(0.70)$ & $0.523(0.13-2.06)$ & 0.353 \\
\hline Bilateral & $17(61 \%)$ & $11(39 \%)$ & $1.18(0.41-3.38)$ & $0.08(0.65)$ & $1.087(0.30-3.90)$ & 0.898 \\
\hline Right & $21(64 \%)$ & $12(36 \%)$ & $1.04(0.37-2.89)$ & $-0.25(0.67)$ & $0.777(0.21-2.87)$ & 0.705 \\
\hline Unspecified & $20(64 \%)$ & $11(36 \%)$ & 1.0 & & & \\
\hline
\end{tabular}

NB: 24 cases of smear negative were excluded from analysis because of missed culture test results 


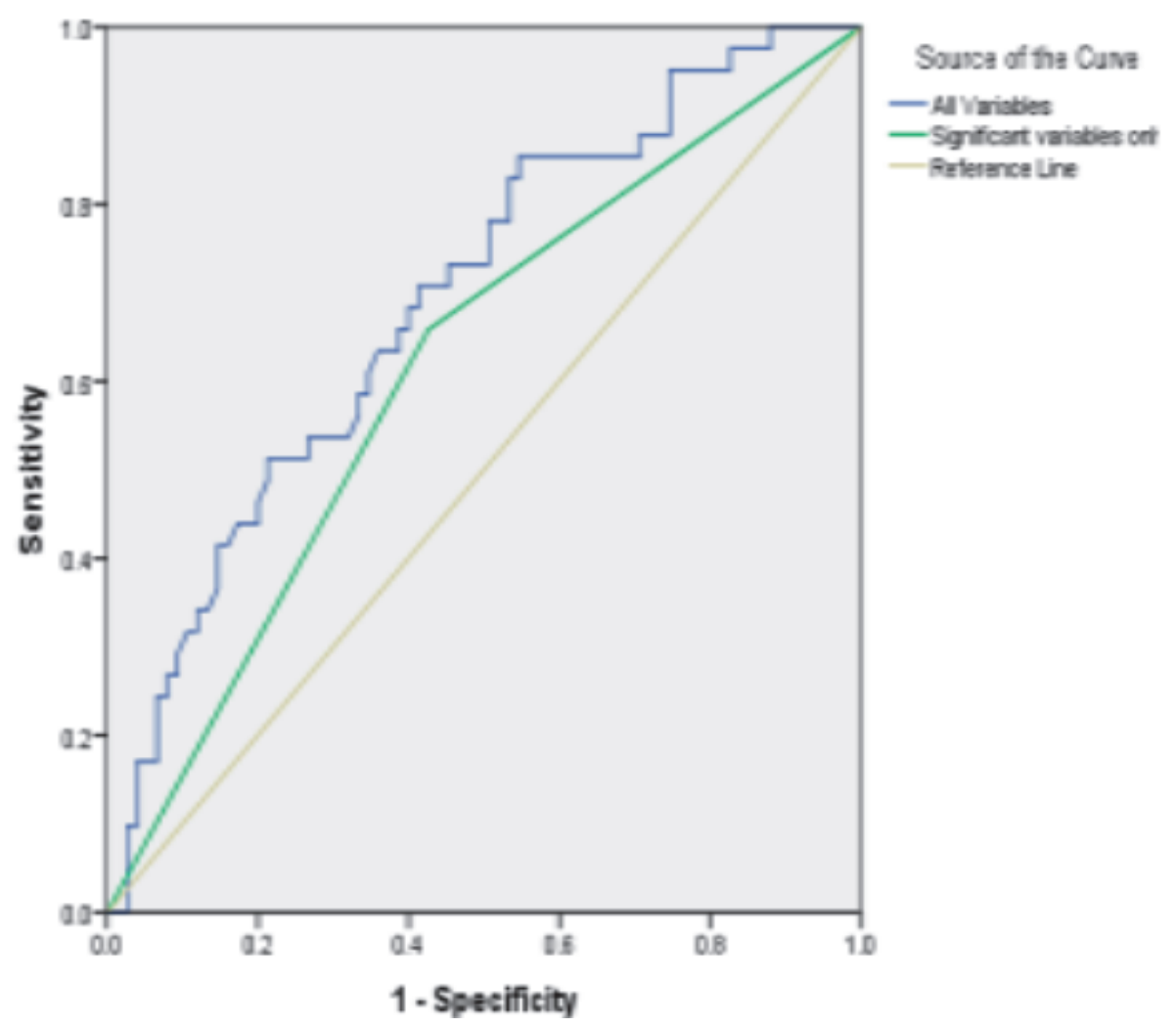

Figure 3. Receiver operator curve characteristics for the prediction of culture-negative from culture-positive among smear-negative TB cases ( $n=146)$.

\section{DISCUSSION}

Our study was designed to evaluate the performance of predictors for estimating the probability of TB in patients suspected of TB and in smear negative cases. $98 \%$ of smear positive TB were confirmed by culture while $64 \%$ of smear negatives were not. The smear test and chest radiography remain the strongest predictors for culture identified TB. Our findings suggest that neither the hallmark symptoms nor radiographic findings are invariably associated with culture-positive compared to culture-negative TB, among smear negative suspects.

The high rates of culture unconfirmed TB in our study confirms the WHO report 2007 and implies that diagnosing smear-negative TB remains problematic even with access to culture and early radiography [32]. A similar phenomenon of culture positivity in smear negative cases has been reported by others where it ranges between $24-62 \%$ [33-35] in different geographical locations. Other studies from high HIV prevalence 
settings using combined clinical and bacteriological case-definitions and not restricted to smear-negative suspects have reported between $10 \%$ and $45 \%$ of patients as culturenegative [36-38]. Furthermore, Nguyen et al. and a Hong Kong based study in the early 1980s $[9,10]$, indicate that culture-negative pulmonary TB is an early disease state with low mycobacterial burden. In addition, a US study of HIV-negative culture-positive patients estimated that negative smears are obtained from $60 \%-80 \%$ of patients with minimal disease, but from only $5 \%-10 \%$ of patients with extensive cavitary lesions [39]. Consequently, we conclude that predictive models based on clinical variables are not useful to discriminate patients with culture negative from culture-positive TB among smears negative cases. This finding suggests that additional indicators are required for predicting TB in patients with a smear and culture negative result.

Many investigators have examined the predictive value of individual signs and symptoms in assessing the risk of TB in clinical suspects [24-28]. Our findings confirm that independent significant variables (positive sputum smear and chest radiography compatible with TB) remain critical elements in the prediction of culture positive TB among patients with clinical suspicion. Scott et al. found that chest radiograph findings consistent with TB was the best available predictor of TB [40], while, Knirsch et al. were successful in using a computer protocol that interpreted chest radiographs to identify high-risk patients for TB [41]. Our study confirms previous reports that identified upper lobe lesions as independent predictive variables of culture-positive TB among clinical suspects [42, 43]. Our findings are also consistent with others who found lesions on lower lung fields were not really related to culture positive TB [44, 45]. Previous studies [46] have also confirmed the reliability of smear microscopy as a proxy for culture in the classification of TB cases. However, the wide confidence interval of positive sputum smear results may indicate the variability in the sensitivity for microscopy $[47,48]$. For example, large variations were reported in the percentage of sputum smear-positive patients among all patients with TB notified to the WHO by sub-Saharan African National Tuberculosis Programs: in 1999, reports ranged from $9 \%$ in Eritrea to $80 \%$ in Benin [49]. This variability may depend on the population tested, technique and skills in getting a good sputum sample, preparation, and reading. Quality assurance is therefore important.

Among smear negative cases only gender was an independent predictor of culturepositive TB. The male predominance in pulmonary TB is well recognized. This has also been shown in reviews in Malaysia, Ireland, Britain and United States [50-54]. So far no undisputed explanation has been forwarded for this finding, thus supporting our hypothesis that among smear-negative cases, culture-negative TB may present itself with no differences in clinical and radiographic abnormalities compared to those with culturepositive TB. Previous studies for the clinical diagnosis of smear negative TB showed also no consistent predictors in the clinical presentation except expectoration, which was identified as a negative predictor [55-57]. Nguyen et al., found a lower frequency of cavitation in culture-negative TB compared with culture-positive subjects $[9,10]$, but it should be emphasized that the differences reported in these previous studies are due to including 
both smear positive and smear negative cases in the groups that were compared with culture negative TB cases. If only smear negative cases would have been used, the results of Nguyen would probably be the same as our results. It is imperative that a high index of suspicion be maintained in order not to miss such cases. This is especially so if the same patient had persistent complaints but without a definitive diagnosis. Hence, a continuing awareness of the clinical spectrum of culture negative TB remains important.

Our study has some limitations. First, the usefulness of our approach is probably limited to African settings with poor performance of sputum smear microscopy and clinically suspected patients relying on chest radiography. The differences in quality of sputum smear microscopy, are likely to have negative impact on the power of other predictors. Second, as there is no gold standard test for the diagnosis of culture negative TB, our study was limited by the lack of absolute certainty that all cases definitely had TB. In particular for pragmatic reasons, clinicians in Ethiopia use all available information for each patient to determine whether TB is indeed present or absent. Accordingly, more insight into clinician's considerations of TB even in case of a negative smear and culture tests results is needed.

Our findings suggest that neither hallmark symptoms nor radiographic findings are invariably associated with culture negative compared to culture positive TB, among smear negative TB cases. It emphasizes that medical providers should not expect a clinical presentation with the characteristic symptoms of advanced TB in order to diagnose and initiate treatment in culture-negative TB. Such awareness is critical, as it is possible to treat culture negative TB, described as an early disease state, based on its likelihood of progression despite the low mycobacterial burden (reviewed in [58]). However, culture negative TB may be more recognized in settings with sufficient resources for extensive work-up to exhaustively exclude other respiratory diseases. For example, Nguyen et al. observed that computer tomography (CT) scans were significantly more frequently performed in culture-negative compared to culture-positive subjects, likely because chest CT scans can reveal abnormalities not seen on chest radiography and can thus aid in diagnosis of culture-negative TB. A prospective consensus diagnostic evaluation study, should be designed to evaluate the added value and ease at which clinical diagnosis of culture-negative TB is made from other possible diagnoses by CT-scan. Since, previous study found that, the clinical judgment of infectious diseases specialists has been shown to be more accurate than prediction models [22].

\section{CONCLUSION}

Our findings suggest that neither the hallmark symptoms nor radiographic findings are invariably associated with culture-negative compared to culture-positive TB, among smear negative suspects. Therefore, we found that a predictive models based on clinical variables will not be useful to discriminate patients with culture negative from culturepositive TB among smears negative cases. 


\section{REFERENCE}

1. Colebunders R, Bastian I. A review of the diagnosis and treatment of smear-negative pulmonary tuberculosis. Int J Tuberc Lung Dis. 2000; 4:97-107.

2. Siddiqi K, Lambert ML, Walley J. Clinical diagnosis of smear-negative pulmonary tuberculosis in lowincome countries: the current evidence. Lancet Infect Dis. 2003; 3:288-96.

3. Corbett EL, Watt CJ, Walker N, et al. The growing burden of tuberculosis: global trends and interactions with the HIV epidemic. Arch Intern Med. 2003; 163:1009-21.

4. Gerhardt G, Natal SR, Pereira A, Lima SF, Penna MLF, Campos HS, Wanke B, Werneck A, Manceau JN: Tuberculose pulmonar semconfirmação bacteriológica [abstract]. J Pneumol 1988, 14:137S.

5. Mello FCQ, Soares SLM, Rezende VMC, Conde MB, Kritski AL, Empirically Treated Tuberculosis - TB: Clinical Profile and Results of Treatment, in AIDS Reference Center - ARC, Riode Janeiro City [abstract]. Tuber Lung Dis 1996, 77:A95.

6. Gordin FM, Slutkin G, Schecter G, Goodman PC, Hopewell PC: Presumptive diagnosis in treatment of pulmonary tuberculosis based on radiographic findings. Am Rev Respir Dis 1989, 139:1090-1093.

7. Centers for Disease Control and Prevention. Reported tuberculosis in the United States, 2013. Atlanta, GA: Centers for Disease Control and Prevention, Services UDoHaH; 2013 May 8, 2015. Report No.

8. Blumberg HM, Burman WJ, Chaisson RE, Daley CL, Etkind SC, Friedman LN, et al. American Thoracic Society/Centers for Disease Control and Prevention/Infectious Diseases Society of America: treatment of tuberculosis. Am J Respir Crit Care Med. 2003; 167(4):603 - 62. doi: 10.1164/rccm.167.4.603 PMID:12588714

9. Nguyen M-VH, Jenny-Avital ER, Burger S, Leibert EM, Achkar JM (2015) Clinical and Radiographic Manifestations of Sputum Culture-Negative Pulmonary Tuberculosis. PLoS (10): e0140003. doi:10.1371/journal.pone.0140003

10. Hong Kong Chest Service/Tuberculosis Research Centre MBMRC. A study of the characteristics and course of sputum smear-negative pulmonary tuberculosis. Tubercle. 1981; 62(3):155- 67. Epub 1981/09/01. PMID: 7032018.ONE 10(10): e0140003. doi:10.1371/journal.pone.0140003

11. Achkar JM, Jenny-Avital ER. Incipient and subclinical tuberculosis: defining early disease states in the context of host immune response. J Infect Dis. 2011; 204 Suppl 4:S1179 - 86. doi: 10.1093/infdis/jir451PMID: 21996700; PubMed Central PMCID: PMC3192549.

12. Cardona PJ. Revisiting the natural history of tuberculosis. The inclusion of constant reinfection, host tolerance, and damage-response frameworks leads to a better understanding of latent infection and its evolution towards active disease. Arch Immunol Ther Exp (Warsz). 2010; 58(1):7 - 14. doi: 10.1007/s00005-009-0062-5 PMID: 20049645.

13. Barry CE 3rd, Boshoff HI, Dartois V, Dick T, Ehrt S, Flynn J, et al. The spectrum of latent tuberculosis: rethinking the biology and intervention strategies. Nature reviews Microbiology. 2009; 7(12):845 - 55. doi: 10.1038/nrmicro2236 PMID: 19855401; PubMed Central PMCID: PMC4144869.

14. Ashba JK, Boyce JM. Undiagnosed tuberculosis in a general hospital. Chest. 1972; 61(5):447 - 51. Epub 1972/05/01. PMID: 4625794.

15. Katz I, Rosenthal T, Michaeli D. Undiagnosed tuberculosis in hospitalized patients. Chest. 1985; 87(6):770 -4. Epub 1985/06/01. PMID: 3996065.

16. Pavi ć I, Radulovi ć P, Bujas T, Peri ć Balja M, Ostoji ć J, Bali č evi ć D. Frequency of tuberculosis at autopsies in a large hospital in Zagreb, Croatia: a 10-year retrospective study. Croat Med J. 2012; 53(1):48 -52. doi: 10.3325/cmj.2012.53.48 PMID: 22351578

17. Edlin GP. Active tuberculosis unrecognised until necropsy. Lancet. 1978; 1(8065):650 - 2. Epub 1978/03/25. PMID: 76179.

18. Linell F, Ostberg G. Tuberculosis in autopsy material, with special reference to cases not discovered until necropsy. Scand J Respir Dis. 1966; 47(3):200 - 8. Epub 1966/01/01. PMID: 5180860.

19. Lee JK, Ng TH. Undiagnosed tuberculosis in hospitalized patients - -an autopsy survey. J R Soc Health. 1990; 110(4):141 - 3. Epub 1990/08/01. PMID: 2121983. 
20. Federal Ministry of Health. Guidelines for Clinical and Programmatic Management of TB, Leprosy and TB/HIV in Ethiopia. Addis Ababa: Federal Minsitry of Health, 2012.

21. Enarson D, Rieder HL, Arnadottir T, Tre'bucq A (2000) Management of Tuberculosis: A guide for Low Income Countries. Paris: International Union Against Tuberculosis and Lung Disease.

22. American Thoracic Society (2000) Diagnostic Standards and Classification of Tuberculosis in Adults and Children. Am J Respir Crit Care Med 161:1376-1395.

23. World Health Organization (2003) Treatment of tuberculosis: guidelines for national programmes, 3rd ed, Geneva, World Health Organization. Available: http://whqlibdoc.who.int/hq/2003/WHO_CDS_TB_ 2003.313_eng.pdf. Accessed 2010 Nov 20.

24. Scott B, Schmid M, Nettleman MD. Early identification and isolation of inpatients at high risk for tuberculosis. Arch Intern Med 1994; 154:326-330

25. Kara S. Rakoczy, MD; Stuart H. Cohen, MD; Hien H. Nguyen, MD. Derivation and Validation of a Clinical Prediction Score for Isolation of Inpatients With Suspected Pulmonary Tuberculosis. Infect Control Hosp Epidemiol 2008; 29:927-932

26. Wisnivesky J, Henschke C, Balentine J, Willner C, Deloire AM, McGinn TG. Prospective validation of a prediction model for isolating inpatients with suspected pulmonary tuberculosis. Arch Intern Med 2005; 165:453-457.

27. Pegues C, Johnson DC, Pegues DA. Implementation and evaluation of an algorithm for isolation of patients with suspected pulmonary tuberculosis. Infect Control Hosp Epidemiol 1996; 17:412-418.

28. Bock NN, McGowan JE, Ahn J. Clinical predictors of tuberculosis as a guide for a respiratory isolation policy. Am J Respir Crit Care Med 1996; 154:1468-1472

29. Riley RD, Hayden JA, Steyerberg EW, Moons KG, Abrams K, Kyzas PA, et al; PROGRESS Group. Prognosis Research Strategy (PROGRESS) 2: prognostic factor research. PLoS Med. 2013;10: e1001380. [PMID: 23393429]

30. Collins GS, Altman DG. Identifying patients with undetected renal tract cancer in primary care: an independent and external validation of QCancer $^{\circledR}$ (Renal) prediction model. Cancer Epidemiol. 2013; 37:11520. [PMID: 23280341]

31. Knirsch CA, Jain NL, Pablos-Mendez A, Friedman C, Hripcsak G. Respiratory isolation of tuberculosis patients using clinical guidelines and an automated clinical decision support system. Infect Control Hosp Epidemiol 1998; 19:94-100.

32. World Health Organization (2007) Improving the diagnosis and treatment of smear-negative pulmonary and extra-pulmonary tuberculosis among adults and adolescents: Recommendations for HIV-prevalent and resource-constrained settings.

33. Deun AV. What is the role of mycobacterial culture in diagnosis and case definition? In: Toman's tuberculosis, Geneva: WHO; 2004; 35-43.

34. Hargreaves NJ, Harries AD, Kemp JR, Kwanjana JH, Salaniponi FM. Smear negative pulmonary tuberculosis: defining better approaches to case finding in Malawi. Malawi Med J 2002; 13: 20-22.

35. Swai FH, Mugusi MF, Mbwambo KJ. Sputum smear negative pulmonary tuberculosis: Sensitivity and specificity of diagnostic algorithm. BMC Res Notes 20121. 4: 475.

36. Apers L, Wijarajah C, Mutsvangwa J, Chigara N, Mason P, et al. (2004) Accuracy of routine diagnosis of pulmonary tuberculosis in an area of high HIV prevalence. Int J Tuberc Lung Dis 8: 945-951.

37. Munyati SS, Dhoba T, Makanza ED, Mungofa S, Wellington M, et al. (2005) Chronic cough in primary health care attendees, Harare, Zimbabwe: diagnosis and impact of HIV infection. Clin Infect Dis 40: 1818-1827.

38. Mutetwa R, Boehme C, Dimairo M, Bandason T, Munyati SS, et al. (2009) Diagnostic accuracy of commercial urinary lipoarabinomannan detection in African tuberculosis suspects and patients.Int J Tuberc Lung Dis. Oct; 13(10):1253-9.

39. Kim T C, Blackman R S, Heatwole K M, Kim T, Rochester D F. Acid-fast bacilli in sputum smears of patients with pulmonary tuberculosis. Am Rev Respir Dis 1984; 129: 264-268.

40. Scott B, Schmid M, Nettleman MD. Early identification and isolation of inpatients at high risk for tuberculosis. Arch Intern Med 1994; 154:326330. 
41. Knirsch CA, Jain NL, Pablos-Mendez A, Friedman C, Hripcsak G. Respiratory isolation of tuberculosis patients using clinical guidelines and an automated clinical decision support system. Infect Control Hosp Epidemiol 1998; 19:94-100.

42. Kanaya AM; Glidden DV; and Chambers HF. Identifying Pulmonary Tuberculosis in Patients With Negative Sputum Smear Results. CHEST 2001; 120:349-355

43. Wisnivesky JP, Serebrisky D, Moore C, Sacks HS, lannuzzi MC, McGinn T. Validity of Clinical Prediction Rules for Isolating Inpatients with Suspected Tuberculosis. J Gen Inten Med 2005; 20:947-952.

44. Greenberg SD, Frager D, Suster B et al .Active pulmonary tuberculosis in patients with AIDS: Spectrum of radiographic findings (including a normal appearance). Radiology 1994; 193: 115-9.

45. Geng E, Kreiswirth B, Burzynski, et al. Clinical and Radiographic correlates of primary and reactivarions tuberculosis: a molecular epidemiology study. JAMA 2005; 293(22):2740-2745

46. Chan $W$ et al. Bacteriological measures for the detection of cases of pulmonary tuberculosis. Bulletin of the World Health Organization, 1971, 45:551-558

47. Steingart KR, Henry M, Ng V, Hopewell PC, Ramsay A, Cunningham J, Urbanczik R, Perkins M, Aziz MA, Pai $M$ : Fluorescence versus conventional sputum smear microscopy for tuberculosis: a systematic review. Lancet Infect Dis 2006, 6:570-581

48. Dorman SE, Chihota VN, Lewis JJ, Shah M, Clark D, Grant AD, Churchyard GJ, Fielding KL: Performance characteristics of the Cepheid Xpert MTB/RIF test in a tuberculosis prevalence survey. PLoS One 2012, 7:e43307

49. World Health Organization. Global tuberculosis control. WHO report 2001. WHO/CDS/TB/2001.287. Geneva, Switzerland: WHO, 2001.

50. O'Reilly P, McDonnell T. The spectrum of Mycobacterial diseases in a Dublin teaching hospital. Irish Med J 1999; 93: 299-302.

51. Howell F, Kelly P, Clancy L. Pulmonary tuberculosis in the Republic of Ireland: an epidemiological profile from a single unit. Respir Med 1990; 84: 111-117.

52. McEvoy M, Maguire H. Tuberculosis in London. J Hosp Infec 1995; 30(S): 296-305.

53. Frieden TR, Sterling T, Pablo-Mendez A et al. The emergence of drug-resistant tuberculosis in New York City. N Eng J Med 1993; 328: 521-6.Miller LG, Asch SM, Yu El et al. A population-based survey of tuberculosis symptoms: How atypical are atypical presentations? Clin Infect Dis 2000; 30: 293-9

54. Samb B, Henzel D, Daley CL, et al. Methods for diagnosing tuberculosis among in-patients in eastern Africa whose sputum smears are negative. Int J Tuberc Lung Dis. 1997;1:25-30.

55. Soto A, Solari L, Diaz J, et al. Validation of a clinical-radiographic score to assess the probability of pulmonary tuberculosis in suspect patients with negative sputum smears. PLoS ONE. 2011;6:e18486.

56. Kanaya AM, Glidden DV, Chambers HF. Identifying pulmonary tuberculosis in patients with negative sputum smear results. Chest. 2001;120:349-55

57. Achkar JM, Jenny-Avital ER. Incipient and subclinical tuberculosis: defining early disease states in the context of host immune response. J Infect Dis. 2011; 204 Suppl 4:S1179 - 86. doi: 10.1093/infdis/jir451 PMID: 21996700; PubMed Central PMCID: PMC3192549.

58. Cobo J, Oliva J, Asensio A, et al. Predicting tuberculosis among HIV infected patients admitted to hospital: comparison of a model with clinical judgment of infectious diseases specialists. Eur J Clin Microbiol Infect Dis 2001; 20:779-784. 

Chapter

Exploratory analyses of the level of salivary Nitric Oxide (NO) metabolites and microbiological outcome in suspected Tuberculosis patients 


\section{ABSTRACT}

Objectives: To examine the diagnostic value of the level salivary NO metabolites in order to assess microbiological outcome (infectiousness), and its association with the severity of the disease (commonly seen as wasting) in TB suspects.

Methods: In this exploratory study, we compared the salivary NO levels in confirmed and unconfirmed TB patients by sputum smear microscopy, chest radiography and molecular GeneXpert tests. Measurements of salivary NO were performed in 90 suspected TB patients. Estimation of salivary $\mathrm{NO}$ was by the measurement of nitrite $\left(\mathrm{NO}_{2}\right)$, employing the Griess reaction according to the method of Green et al. [21]. The nitrite levels in the saliva were determined from the universal color change, constructed from the known standard concentration.

Results: We found that, there is a significantly higher proportion of low salivary NO levels $(P<0.019)$ in patients positive for smear (smear positive $T B$ ) than among negatives for smears. Surprisingly, among smear negative cases, we did not observe a significant difference in salivary NO levels (0.086) between the TB-cases and non-TB-cases. Interestingly, in our exploratory study no significant correlation between BMI and NO levels was found.

Conclusions: The results of our findings support our hypothesis that, patients unable to respond with an effective production of salivary $\mathrm{NO}$ metabolites in response to $\mathrm{Myco-}$ bacterium Tuberculosis infection, are more likely to be associated with increased risk of acquiring TB. Interestingly, in our exploratory study no significant correlation between $\mathrm{BMI}$ was found on the groups compared and with regards to the levels of salivary NO. Hence we could not support our second hypothesis that levels of salivary NO could be associated with the severity of the TB. 


\section{INTRODUCTION}

The diagnosis of tuberculosis (TB) in resource-poor endemic regions where $95 \%$ of cases and $98 \%$ of TB deaths [1] occur is challenging. In these settings, diagnosis still depends on sputum smear microscopy [2], even though accuracy is suboptimal and fails to diagnose one-third to half of all TB patients [3]. In addition, cases are diagnosed based upon passive case finding, and it is likely that a large proportion of these patients will be misdiagnosed.

In the Global TB Report 2014, the World Health Organization (WHO) declared that "one of the most urgently needed tests is a rapid biomarker-based test that can diagnose TB" [4]. Ideally, such TB biomarker should be able to detect the earliest and most difficult to diagnose TB cases, and should be suitable for use in resource-limited settings. Previous studies show that the presence of nitric oxide (NO) and its metabolites are potential screening biomarkers for TB $[5,6]$, but its relative importance is debated $[7,8]$. Patients with TB were reported to have lower levels of exhaled NO and urinary NO metabolites, compared to healthy controls [6, 9, and 10]. However, there is very limited evidence on NO in direct diagnosis of TB [11].

Human mouth represents the environment with a constant supply of NO metabolites from L-arginine. The production of a stable salivary NO metabolites, may be affected during nutritional deficiencies, a common condition in TB suspects [12, 13]. Several studies reported that patients with active TB are more likely to experience wasting, and have a lower body mass index $(\mathrm{BMI}=\mathrm{kg} / \mathrm{m} 2)$ than healthy individuals [14-17]. Therefore, it is hypothesized that, patients unable to respond with an effective production of salivary NO metabolites in response to Mycobacterium Tuberculosis infection, are more likely to have severe (commonly seen as wasting in TB suspects) and more infectious forms of TB.

NO can be measured directly in the exhaled air [18-20] as well as by the stable urinary metabolites, nitrite and nitrate [19]. In recent years there has been much interest to investigate the level and especially the role of urinary and exhaled air NO in relation to host defense in human TB $[20,21]$. However, there is a lack of clinical studies describing the level of salivary NO in suspected TB patients in the diagnostic phase. Therefore, in this study, the diagnostic value of the level salivary NO metabolites was examined to assess microbiological outcome (infectiousness), and its association with the severity of the disease (commonly seen as wasting) in TB suspects. 
Chapter 5

\section{METHODS}

\section{Study population and methods}

In this prospective study, we compared the salivary NO levels in confirmed and unconfirmed suspected TB patients by sputum smear microscopy, chest radiography and molecular GeneXpert tests. Measurements of salivary NO were performed at the Kilifi County Hospital and Malindi District Hospital, which serve as both referral centers and primary-care facilities in the coastal region of Kenya. Kilifi County Hospital is a 172-bed hospital on the eastern coast of Kenya, serving an area of $3870 \mathrm{~km} 2$ with a population of approximately 500,000 people [22]. Whereas, the Malindi District Hospital is a 208-bed hospital situated on the same coast $60 \mathrm{~km}$ north of Kilifi town [23].

The study protocol was reviewed and approved by the Ethical Review Committee of Pwani University (ERC/MSc/026/2015), and authorization to conduct this study at the hospitals was given by the Kilifi County Research Ethical Review Committee. Patients were asked to read and sign the informed consent form for the collection of samples and subsequent analysis. These were available in both English and Swahili languages, and an interpreter was available if required. Personal identifiers were not collected and data were analysed anonymously.

All consenting patients aged $\geq 18$ years, consecutively presenting themselves at the hospital with symptoms compatible with TB, were considered eligible for inclusion. The patients were screened for TB in accordance with the guidelines for Management of Tuberculosis and Leprosy in Kenya [24], between 23 June and 22 August 2015. Exclusion criteria were current anti-TB treatment. All participants were interviewed by treating clinicians using a pre-coded structured questionnaire on demographics, TB signs and symptoms and factors potentially affecting salivary NO levels, i.e., smoking history, recent alcohol consumption, history of antibiotic treatment, antibacterial mouthwash, HIV status and recent consumption of nitrate-rich vegetables and caffeine-rich tea.

\section{NO estimation in the serum and saliva}

After the interview, salivary NO levels were analyzed using commercially available NO Test Strips (manufactured by Berkeley Test, USA). The NO Test Strip is a simple, noninvasive procedure that can be performed by any individual in remote setting with no established diagnostic methods. Estimation of salivary NO was by the measurement of nitrite $\left(\mathrm{NO}_{2}\right)$, employing the Griess reaction according to the method of Green et al. [25]. The strip is administered on the tongue of the patient for no longer than 5 seconds, for the absorption pad to saturate. The nitrite levels in the saliva were determined from the universal color change (Figure 1) constructed from the known standard concentration. 


\section{DEPLETED}

\section{NO-poor diet}

\section{LOW}

\section{THRESHOLD \\ NO-rich diet \\ TARGET}

\section{HIGH}

Figure 1. Color change seen depending upon patients' salivary NO level.

\section{Principle of the Griess reaction}

The Griess reaction involves formation of a chromophore during the reaction of nitrite with sulfanilamide and heterocyclic amine of naphthylene-ethylene-diamine (Griess reagent) under low $\mathrm{pH}$ conditions. During this reaction, acidified nitrite undergoes diazotization with sulfanilamide to form a diazonium salt. This diazonium salt then couples to $\mathrm{N}$-(1-Naphthyl) ethylenediamine to form a magenta-colored azo dye with a characteristic absorption spectrum. The only stable product formed by spontaneous autoxidation of $\mathrm{NO}$ in oxygenated solutions is $\mathrm{NO}_{2}$.

The chemistry of the Berkeley Test Nitric Oxide Test Strips involves the conversion of $\mathrm{NO}_{2}$ to a deep purple azo compound, producing a readily observable colour change that enables accurate determination of NO concentration [25]. These strips can detect between 21 and $869 \mu \mathrm{M}$ of $\mathrm{NO}_{2}( \pm 15 \mu \mathrm{M})$. NO levels have been categorized as low, normal and high, which correlates to specific concentrations of $>21,108-217$ and 434-869 $\mu \mathrm{M}$, respectively [26]. Typical NO levels in human saliva range from 10 to $1000 \mu \mathrm{M}$, therefore these strips are sufficient to detect the NO levels present in most samples [27].

\section{Data analysis}

Data were analyzed using SPSS version 20 (SPSS Inc., Chicago, IL, USA). Descriptive categorical variables are summarized as proportions, whereas continuous variables as means \pm standard deviations. The $\chi 2$ test was used to determine the difference in proportions. Analysis of differences between diagnostic outcome and levels of NO was performed using the $\chi 2$ test and Fisher's exact test for categorical variables and Student's t test for continuous variables where appropriate. The salivary NO levels were stratified for known influencing factors which had a distinctly different distribution between the patient groups. All tests with a p-value less than 0.05 are considered statistically significant. 


\section{RESULTS}

We recruited 90 suspected TB patients during a two-month period from June to August 2015 (Figure 2), of which, 24 (27\%) were diagnosed as smear-positive TB. The remaining 66 (73\%) suspected TB patients had negative smear results, of which 41 (46\%) subsequently received a diagnosis by chest radiography as smear-negative TB if the lesions were highly consistent for TB. However, in only 14 (52\%) of the smear negative TB cases, TB diagnosis was confirmed by the molecular Xpert MTB/RIF test (new molecular test for detecting the presence of TB bacteria).

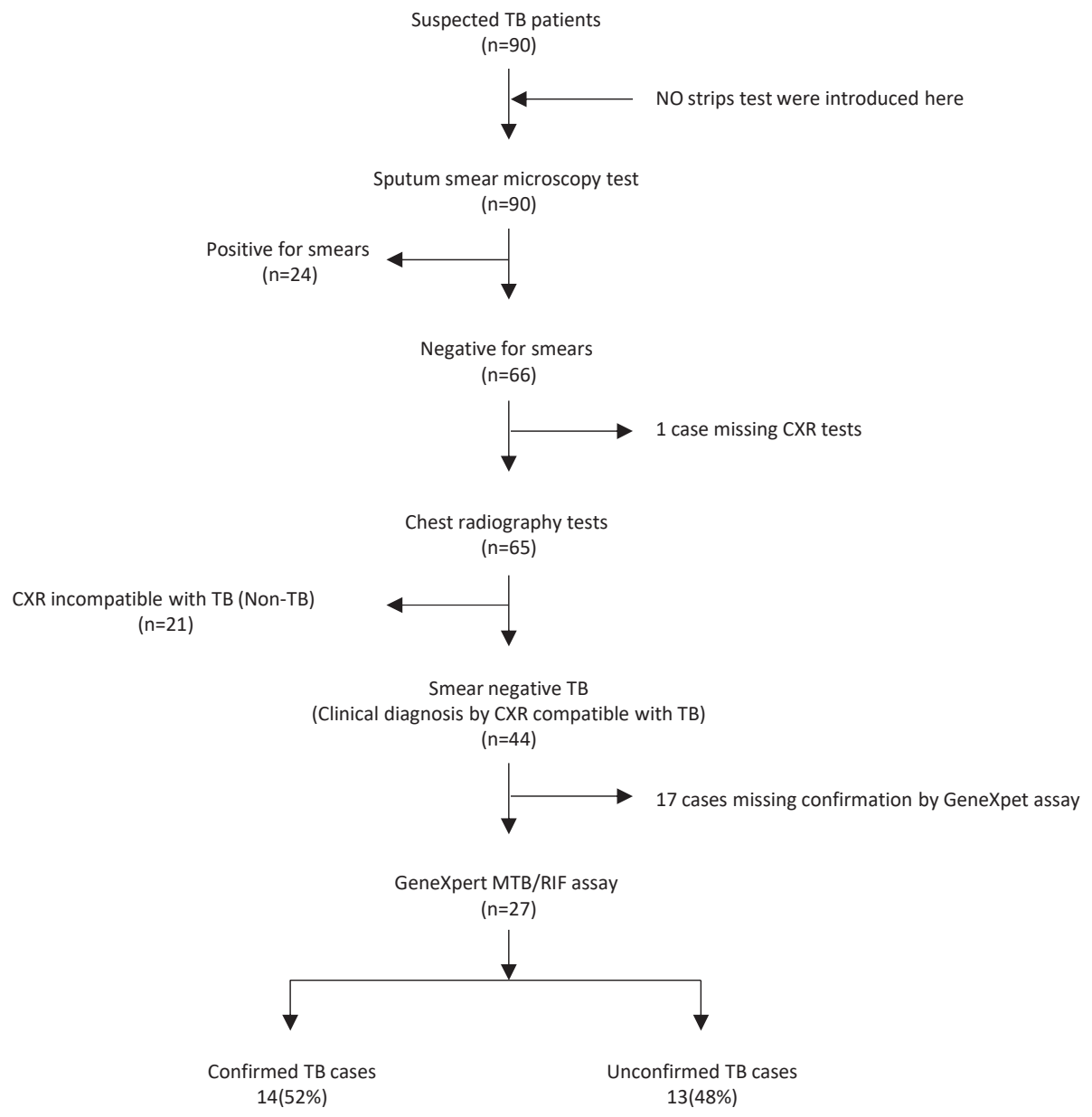

Figure 2. Patient flowchart in the diagnostic process and the point at which NO test strips may be used to measure salivary NO levels. 
Clinical parameters and salivary NO levels between different groups of patients are given in Table 1.Almost all variables showed no significant difference between patients positive (smear-negative TB) compared to negative (non-TB cases) for chest $x$-ray lesions compatible with TB. However, a significant difference in age was observed between patients positive for smear compared to negative for smear TB. In addition, the level of salivary NO in cases positive for smear (smear positive TB cases) was significantly $(P<0.019)$ lower than among negative for smears. Although we did not observe a significant difference in salivary $\mathrm{NO}$ levels $(P=0.086)$ between patients smear-negative TB compared to non-TB cases, but there was a larger proportion of patients with high levels of NO in the smear-negative TB group. Furthermore, no significant correlation between BMI was found on all the groups compared.

Table 1. Clinical parameters and salivary Nitric oxide levels between different groups of patients

\begin{tabular}{|c|c|c|c|c|c|c|}
\hline & \multicolumn{2}{|c|}{ Smear sputum microscopy results } & \multirow{2}{*}{ P value } & \multirow{2}{*}{$\begin{array}{l}\text { Chest X-ray } \\
\text { Compatible with TB } \\
\text { (Smear negative } \\
\text { TB) } \\
(n=44)\end{array}$} & \multirow{2}{*}{$\begin{array}{l}\text { findings } \\
\text { B Not compatible } \\
\text { with TB } \\
\text { (No TB) } \\
(n=21)\end{array}$} & \multirow[b]{2}{*}{$P$ value } \\
\hline & $\begin{array}{l}\text { Smear positive } \\
\text { TB } \\
(n=24)\end{array}$ & $\begin{array}{l}\text { Not detected by } \\
\text { smears } \\
(n=66)\end{array}$ & & & & \\
\hline Sex (Male) & $16(67 \%)$ & $31(47 \%)$ & 0.098 & $20(46 \%)$ & $10(48 \%)$ & 1.000 \\
\hline Age & $33.9(12.2)$ & $42.7(17.2)$ & 0.023 & $42.4(16.7)$ & $43.2(19.2)$ & 0.879 \\
\hline Body mass index & $19.8(2.8)$ & $21.0(4.4)$ & 0.228 & $20.8(3.5)$ & $20.8(4.7)$ & 0.978 \\
\hline \multicolumn{7}{|l|}{ HIV status } \\
\hline Positive & $7(29 \%)$ & $20(30 \%)$ & 0.230 & $12(27 \%)$ & $6(29 \%)$ & 0.794 \\
\hline Negative & $17(71 \%)$ & $39(59 \%)$ & & $28(64 \%)$ & $12(57 \%)$ & \\
\hline Unknown & - & $7(11 \%)$ & & $4(9 \%)$ & $3(14 \%)$ & \\
\hline \multicolumn{7}{|l|}{ Salivary NO levels: } \\
\hline Low & $15(63 \%)$ & $25(38 \%)$ & 0.019 & $18(41 \%)$ & $10(48 \%)$ & 0.086 \\
\hline Normal & $8(33 \%)$ & $20(30 \%)$ & & $11(25 \%)$ & $9(43 \%)$ & \\
\hline High & $1(4 \%)$ & $21(32 \%)$ & & $15(34 \%)$ & $2(9 \%)$ & \\
\hline
\end{tabular}

HIV Human Immunodeficiency Virus, NO nitric oxide

The relationship between associated factors that may impair the salivary NO levels in suspected TB patients is presented in table 2. No effect of the nitrate/arginine-rich diet consumption was found to affect the salivary NO levels, and the same goes for known confounders like alcohol, recent antibiotic treatment or antibacterial mouthwash use. The analysis yielded $(P<0.012)$ gender, and $(P<0.041)$ smoking as significant factors that impaired the level of salivary NO in suspected TB patient. 
Chapter 5

Table 2. Relationship between associated factors and NO levels in patients suspected of having TB

\begin{tabular}{|c|c|c|c|c|}
\hline & & NO levels & & \\
\hline & $\begin{array}{l}\text { Low } \\
n=40(\%)\end{array}$ & $\begin{array}{l}\text { Normal } \\
n=28(\%)\end{array}$ & $\begin{array}{l}\text { High } \\
n=22(\%)\end{array}$ & $P$ value \\
\hline \multicolumn{5}{|l|}{ Gender } \\
\hline Male & $18(45)$ & $21(75)$ & $8(36)$ & 0.012 \\
\hline Female & $22(55)$ & $7(25)$ & $14(64)$ & \\
\hline Age & $41(18.1)$ & $40.3(13.3)$ & $39.2(17.4)$ & 0.922 \\
\hline Body Mass Index & $20.7(4.4)$ & $20.3(3.4)$ & $21.2(4.2)$ & 0.735 \\
\hline \multicolumn{5}{|l|}{ History of anti-TB } \\
\hline New & $40(100)$ & 26(93) & 19(86) & 0.073 \\
\hline Relapse & - & $2(7)$ & $3(14)$ & \\
\hline \multicolumn{5}{|l|}{ Alcohol status } \\
\hline Alcohol & $4(10)$ & $4(14)$ & $2(9)$ & 0.808 \\
\hline No Alcohol & $36(90)$ & $24(86)$ & 20(91) & \\
\hline \multicolumn{5}{|l|}{ Smoking status } \\
\hline Smoking & $2(5)$ & $7(25)$ & 2(9) & 0.041 \\
\hline Non-smoking & $38(95)$ & $21(75)$ & 20(91) & \\
\hline \multicolumn{5}{|l|}{ Diet consumption } \\
\hline Nitrate-/Arginine-rich diet & $35(88)$ & $24(86)$ & $18(82)$ & 0.830 \\
\hline No Nitrate-/Arginine-rich diet & $5(12)$ & $4(14)$ & $4(9)$ & \\
\hline \multicolumn{5}{|l|}{ Antibiotic status } \\
\hline Antibiotic treatment & $27(68)$ & $18(64)$ & $9(41)$ & 0.106 \\
\hline No Antibiotic treatment & $13(32)$ & $10(36)$ & 13(59) & \\
\hline \multicolumn{5}{|l|}{ HIV status } \\
\hline Positive & $10(25)$ & $11(39)$ & $6(27)$ & 0.776 \\
\hline Negative & $27(68)$ & $15(54)$ & $14(64)$ & \\
\hline Unknown & $3(7)$ & $2(7)$ & $2(9)$ & \\
\hline
\end{tabular}

\section{DISCUSSION}

The results of our findings support our hypothesis that, patients unable to respond with an effective production of salivary NO metabolites in response to Mycobacterium Tuberculosis infection, are more likely to be associated with increased risk of acquiring TB. Thus, we found that, there is a significantly higher proportion of low salivary NO levels in patients positive for smear (smear positive TB) than among negative for smears. Surprisingly, among smear negative cases, we did not observe a significant difference in salivary NO levels (0.086) between the TB-cases and non-TB-cases. Similarly, a previous study also found no difference in NO levels between positive and negative chest radiography findings at treatment initiation [6]. Although our exploratory analyses of salivary 
NO levels were restricted only to TB suspects, our findings are similar to Idh et al., [6] who observed an association between a low level of exhaled NO with smear positive TB compared to control healthy subjects. It should be emphasized that previously reported differences may potentially introduce bias due to comparing TB cases and healthy controls than if the analysis is restricted only to TB suspects.

Weight is a well-recognized measure of TB severity and treatment response [28]. Several studies reported that patients with active TB are more likely to experience wasting, and have a lower BMI (BMI=kg/m2) than healthy individuals [14-17]. This may lead to deficiencies of important nutrients, such as the amino acid L-arginine, which is essential for NO production. Interestingly, in our exploratory study no significant correlation between BMI and NO levels was found. Hence we could not support our second hypothesis that levels of salivary NO could be associated with BMI and thus indirectly with the severity of the TB. This finding is in line with the study of Idh et al., [6] in which they neither found an association between BMI and $\mathrm{NO}$ levels at treatment initiation. However, larger clinical studies, including long term clinical follow up will be essential to test this hypothesis thoroughly.

The present investigation is limited in some aspects, such as the small sample size. Thus, further longitudinal studies must be undertaken involving a larger number of subjects to evaluate the role of salivary NO in the screening of suspected TB patients. In addition, our analysis yielded $(P<0.012)$ gender and $(P<0.041)$ smoking as significant factors to influence on the level of salivary NO in TB patients. Thus, these factors may have obscured TB-related differences in salivary NO levels among TB patients. The results of our study cannot explain the reason underlying the difference in NO levels between males and females. However, it has been speculated that males might not be as susceptible as females in the production of NO [29-31], due to low endothelial responsiveness to the circulating adipocytokines produced by visceral fat tissue. However, previous studies reported higher NO production in female than males, despite the controversy over the differences [32,33]. Finally, a possible explanation for the effects of smoking on NO levels may be the oxidative burden, a process that stimulates the production of NO [34, 35].

A series of reviews has shown that biomarkers such as adenosine deaminase, interferon-gamma (IFN- - ) [36-39] and NO have the potential for screening TB suspects [5, 6]. These biomarkers, especially salivary NO, are easy to measure and inexpensive. However, there is the potential downside that these biomarkers are not necessarily TBspecific, a weakness that may be addressed by the identification of biosignaturesmultiple signals driven by specific host-pathogen interaction [40, 41]. Therefore, this makes the determination of a single host biomarker's sensitivity and specificity challenging. So far only a few studies have integrated combinations of markers to predict treatment outcome [42]. Consequently, uncertainties exist for the use of single biomarkers to diagnose infection status prior to initiation of therapy, and differentiate recent or progressive infection from and distant or latent infection. Inability to distin- 
guish these two states will greatly reduce the potential for these tests to add value to clinical decision-making in TB-endemic regions, where a majority of adults would be expected to have been exposed to Mycobacterium tuberculosis. This is particularly important where laboratory services are being overwhelmed with demand for smear microscopy. Despite these limitations, a better prospective study should be designed to confirm our findings in various populations of suspected TB patients in order to determine if and how salivary NO strip test may be routinely used by admitting physician to improve the adequacy of current diagnostic tests.

\section{CONCLUSION}

The results of our findings support our hypothesis that, patients unable to respond with an effective production of salivary NO metabolites in response to Mycobacterium Tuberculosis infection, are more likely to be associated with increased risk of acquiring TB. Our findings and previous studies suggest that the use of NO metabolites as a biomarkers may have great value for screening of smear positive TB. Interestingly, in our exploratory study no significant correlation between BMI was found on the groups compared and with regards to the levels of salivary NO. Hence we could not support our second hypothesis that levels of salivary NO could be associated with the severity of the TB. 


\section{REFERENCES:}

1. Raviglione MC, Gupta R, Dye CM, Espinal MA: The burden of drug-resistant tuberculosis and mechanisms for its control. Ann N Y Acad Sci 2001 953:88 - 97.

2. WHO: Global Tuberculosis Report. World Health Organization 2012.

3. Steingart, K.R., Ng, V., Henry, M., Hopewell, P.C., Ramsay, A. Cunningham, J., Urbanczik, R., Perkins, M.D., Aziz, M.A., Pai, M. Sputum Processing methods to improve the sensitivity of smear microscopy for tuberculosis: a systematic review. Lancet Infectious Diseases. 2006. 6: 664-674.

4. Global tuberculosis report 2014. Geneva, Switzerland: World Health Organization, 2014.

5. Van Beek SC, Nhung NV, Sy DN, Sterk PJ, Tiemersma EW, Cobelens FG. Measurement of exhaled nitric as a potential screening tool for pulmonary tuberculosis. Int J Tuberc Lung Dis 2011; 15 (2): 185-92.

6. Idh J, Westman A, Elias D, Moges F, Getachew A, Gelaw A, Sundqvist T, Forslund T, Alemu A, Ayele B, Diro E, Melese E, Wondmikun Y, Britton S, Stendahl O, Schön T. Nitric oxide production in the exhaled air of patients with pulmonary tuberculosis in relation to HIV co-infection. BMC Infectious Diseases 2008; $8: 146$.

7. Chan ED, Chan J, Schluger NW. What is the role of nitric oxide in murine and human host defense against tuberculosis? Am J Respir Cell Mol Biol 2001; 25:606e12.

8. Stenger S. Cytolytic T cells in the immune response to mycobacterium tuberculosis. Scand J Infect Dis 2001; 33(7):483e7.

9. Schön T et al. Effects of a food supplement rich in arginine in patients with smear positive pulmonary tuberculosis; a randomized trial. Tuberculosis 91 (2011) 370 e377

10. Wang CH, Liu CY, Lin HC, Yu CT, Chung KF, Kuo HP: Increased exhaled nitric oxide in active pulmonary tuberculosis due to inducible NO synthase up regulation in alveolar macrophages. European Respiratory Journal 1998, 11:809-815.

11. Pal R, Dahal S, Gurung A. Exhaled breath analysis in tuberculosis case detection: The new horizon. Nepal J Epidemiol 2013; 3(2): 243-4.

12. Peters, W. H. and Lubs, H. (1987) Serum free amino acids of healthy males and pregnant women from Ethiopia. Die Nahrung 31, 923-5.

13. Zea, A. H., Culotta, K. S., Ali, J., Mason, C., Park, H. J., Zabaleta, J., Garcia, L. F., Ochoa, A. C. (2006) Decreased expression of CD3zeta and nuclear transcription factor kappa B in patients with pulmonary tuberculosis: potential mechanisms and reversibility with treatment. J Infect Dis 194, 1385-93

14. Van Lettow M, Kumwenda JJ, Harries AD, et al. Malnutrition and the severity of lung disease in adults with pulmonary tuberculosis in Malawi. Int J Tuberc Lung Dis. 2004; 8:211-217

15. van Lettow M, Fawzi WW, Semba RD. Triple trouble: the role of malnutrition in tuberculosis and human immunodeficiency virus co-infection. Nutr Rev. 2003; 61:81-90.

16. Zachariah R, Spielmann MP, Harries AD, Salaniponi FM. Moderate to severe malnutrition in patients with tuberculosis is a risk factor associated with early death. Trans R Soc Trop Med Hyg. 2002; 96:291-294

17. Tverdal A. Body mass index and incidence of tuberculosis. Eur J Respir Dis. 1986; 69:355-362. [PubMed]

18. WHO. Treatment of tuberculosis: guidelines, whqlibdoc.who.int/hq/2003/WHO_CDS_TB_2003.313_eng.pdf; 2008. WHO/CDS/TB 2003.313.WHO classification.

19. Verdon CP, Burton BA, Prior RL. Sample pre-treatment with nitrate reductase and glucose-6-phosphate dehydrogenase quantitatively reduces nitrate while avoiding interference by NADPp when the griess reaction is used to assay for nitrite. Anal Biochem 1995; 224:502e8.

20. Chan ED, Chan J, Schluger NW. What is the role of nitric oxide in murine and human host defense against tuberculosis? Am J Respir Cell Mol Biol 2001; 25:606e12.

21. Wang CH, Liu CY, Lin HC, Yu CT, Chung KF, Kuo HP. Increased exhaled nitric oxide in active pulmonary tuberculosis due to inducible NO synthase upregulationin alveolar macrophages. Eur Respir J 1998;11(4):809e15.

22. KDH Trust (2015). Friends of Kilifi District Hospital Info Page. [Internet]. Available from: www.kdhfriends.org 


\section{Chapter 5}

23. Elective Africa. May 2015. [Internet]. Available from electiveafrica.com/ destinations/Malindi

24. MoH. Guidelines for Management of Tuberculosis and Leprosy in Kenya Nairobi, July 2013.

25. Green LC, Wagner DA, Glogowski J, Skipper PL, Wishnok JS, Tannenbaum SR. Analysis of nitrate, nitrite, and [15N] nitrate in biological fluids. Anal Biochem 1982; 126:131-8.

26. Jules, M. Berkeley Test Information_TB. info@berkeleytest.com; 2015

27. McKnight G.M., Smith L.M., Drummond R.S., Duncan C.W., Golden M., Benjamin, N. Chemical synthesis of nitric oxide in the stomach from dietary nitrate in humans. Gut. 1997. 40; $2: 211-214$.

28. Khan A, Sterling TR, Reves R, Vernon A, Horsburgh CR. Lack of weight gain and relapse risk in a large tuberculosis treatment trial. Am J Respir Crit Care Med 2006; 174:344-8

29. Fujita K, Wada K, Nozaki Y, Yoneda M, Endo H, Takahashi H, et al. Serum nitric oxide metabolite as a biomarker of visceral fat accumulation: clinical significance of measurement for nitrate/nitrite. Med Sci Monit. 2011; 17: CR123 - 131.

30. Kondo T, Ueyama J, Imai R, Suzuki K, Ito Y. Association of abdominal circumference with serum nitric oxide concentration in healthy population. Environmental Health and Preventive Medicine.2006; 11: 321 -325 .

31. Higashi Y, Sasaki S, Nakagawa K, Kimura M, Noma K, Hara K, et al. Low body mass index is a risk factor for impaired endothelium-dependent vasodilation in humans: role of nitric oxide and oxidative stress. J Am Coll Cardiol. 2003; 42: 256-263.

32. Ghasemi A, Zahedi AsI S, Mehrabi Y, Saadat N, Azizi F. Serum nitric oxide metabolite levels in a general healthy population: relation to sex and age. Life Sci. 2008; 83: 326 - 331.

33. Ghasemia A, Zahediasl S, Azizi F. Elevated nitric oxide metabolites are associated with obesity in women. Arch Iran Med. 2013; 16(9): 521-525.Johnson GK, Hill M. Cigarette smoking and the periodontal patient. J Periodontol 2004; 75:196-209.

34. Razali M, Palmer RM, Coward P, Wilson RF. A retrospective study of periodontal disease severity in smokers and non-smokers. Br Dent J 2005; 198:495-8

35. Goto M, Noguchi Y, Koyama H, Hira K, Shimbo T, et al. (2003) Diagnostic value of adenosine deaminase in tuberculous pleural effusion: A meta-analysis. Ann Clin Biochem 40: 374-381.

36. Greco S, Girardi E, Masciangelo R, Capoccetta GB, Saltini C (2003) Adenosine deaminase and interferon gamma measurements for the diagnosis of tuberculous pleurisy: A meta-analysis. Int J Tuberc Lung Dis 7: 777-786.

37. Jiang J, Shi HZ, Liang QL (2007) Diagnostic value of interferon-g in tuberculous pleurisy: A meta-analysis. Chest 131: 1133-1141.

38. Liang QL, Shi HZ, Wang K, Qin SM, Qin XJ (2008) Diagnostic accuracy of adenosine deaminase in tuberculous pleurisy: A metaanalysis. Respir Med 102: 744-754.

39. Trajman A, Pai M, Dheda K, van zyl Smit R, Zwerling A, et al. (2008) Novel tests for diagnosing tuberculous pleural effusion: What works and what does not? Eur Respir J 31: 1098-1106.

40. Jacobsen $\mathrm{M}$ et al. (2008). Novel strategies to identify biomarkers in tuberculosis. Biological Chemistry, 389(5):487-495.

41. Walzl G et al. (2008). Biomarkers for TB treatment response: challenges and future strategies. The Journal of Infection, 57(2):103-109. Epub 2008 Jul 22.

42. Wallis RS et al. (2000). Predicting the outcome of therapy for pulmonary tuberculosis. American Journal of Respiratory and Critical Care Medicine, 161:1076-1080. 
Chapter

General Discussion 



\section{INTRODUCTION}

In this chapter, the main findings with regards to the research questions are summarized and general conclusions based on the findings of the studies presented in this thesis are described. Furthermore, the strength and limitations of this thesis are considered and suggestions for further research into quality of TB diagnostic care are presented. This chapter concludes with recommendations for three categories of stakeholders: TB service providers, program managers and policy makers.

The main research finding is that culture-negative TB cannot be discriminated from culture-positive TB among smear negative suspects. Thus supporting our hypothesis that culture-negative TB may present with no differences in clinical and radiographic abnormalities compared to those with culture-positive TB among smear-negative cases. Our findings further emphasize that in settings like ours with higher TB prevalence, there is a low threshold for starting antituberculous therapy, especially in patients with radiographic lesions compatible with $\mathrm{TB}$, despite negative culture results. However, we confirmed that independent significant variables (positive sputum smear and chest radiography compatible with $\mathrm{TB}$ ) remain critical elements in the prediction of culture positive TB among patients with clinical suspicion. Furthermore, the results of our findings support our hypothesis that patients unable to respond with an effective production of salivary NO metabolites in response to Mycobacterium Tuberculosis (Mtb) infection, are more likely to be associated with increased risk of acquiring TB. Our findings and previous studies suggest that the use of NO metabolites as a biomarkers may have great value for screening of smear positive TB. Finally, our study indicates that the degree of trust that TB service providers have in the diagnostic test results have a great impact on compliance with guidelines. However, a specific aspect of our findings was that TB service providers had developed negative expectations about the results of sputum smear microscopy tests.

Our findings suggest that neither the hallmark symptoms nor radiographic findings are invariably associated with culture-positive compared to culture-negative TB, among smear negative suspects. This confirms the findings of a previous study which reported that diagnosing smear-negative TB remains problematic even with access to culture and early radiography [1]. Considering that, other studies using combined clinical and bacteriological case-definitions reported between $10 \%$ and $45 \%$ of patients as culturenegative TB [2-4]. Most importantly, high rates of late diagnoses and low rates of culture confirmed TB were reported [1]. This is particularly common in real practice due to a number of obstacles intrinsic to the clinical setting [5]. First, in the majority of patients with a negative smear- and culture result, their illness is commonly in its early stage, when 'red flag' symptoms are yet to evolve and bacteriological confirmation is often difficult if TB is not advanced [6-8]. Second, the time to detection and speciation of cultures, may be up to 7 weeks, particularly when the burden of the mycobacteria is very low, as is often the case in smear and culture-negative TB [9]. All of these factors 
considered, emphasizes that medical providers should not expect a clinical presentation with the characteristic symptoms of advanced TB in order to diagnose and initiate treatment in culture-negative TB. Such awareness is critical, as it is possible to treat culture negative TB, described as an early disease state, based on its likelihood of progression despite the low mycobacterial burden (reviewed in [10]). This is particularly important to policy-makers and from medical personnel to volunteer health workers, given that in resource-limited settings TB is often diagnosed by non-physicians, who have low TB knowledge and little experience [11].

To improve the accuracy of diagnostic work-up several new diagnostic tests have been suggested [12-16]. Ideally, a single reference test to classify culture negative TB is preferred. However, such a single and error-free test, also known as a reference or "gold" standard, is not available [17]. This is problematic, as errors in the final disease classification can seriously bias the results from correct diagnosis $[17,18]$. In addition, clinical definitions of culture-negative TB in existing guidelines are rather vague [19-21] and do not allow to classify patients according to their probability of TB. Our findings confirm that independent significant variables (positive sputum smear and chest radiography compatible with $\mathrm{TB}$ ) remain critical elements in the prediction of culture positive TB among patients with clinical suspicion. Our finding is in agreement with a previous study [22] that confirmed the reliability of smear microscopy as a proxy for culture in the classification of TB cases. Therefore, in countries with a high prevalence of TB, the specificity of smear microscopy may thus be superior to that of culture. This may be true even for the diagnosis of TB [23], since acid-fast bacillus (AFB) demonstrated in direct sputum smears would then almost invariably represent $M$. tuberculosis, even in areas with a high burden of HIV [24]. By contrast, in countries with a low prevalence of $\mathrm{TB}$, culture (or alternative techniques of species identification) will often be indispensable to the differentiation of TB from other mycobacterial diseases. As prevalence falls, clinicians will be less likely to suspect TB, and will be less likely to be experts on recognizing TB, so that even a late culture result will be useful. Although, other sensitive rapid diagnostics have shown great promise when evaluated against culture [25], these tests still need to be evaluated for their usefulness in diagnosing culture negative TB. Diagnostic barriers will not be completely resolved until rapid diagnostic methods with sensitivity approaching $100 \%$ become widely available [26].

The Xpert MTB/RIF test ( new molecular test for detecting the presence of Mycobacterium TB) for diagnosing TB increases the number of true cases diagnosed, and reduces the number of false-positive cases $[27,28]$ and is the preferred diagnostic test for use after screening with chest radiography. This screening should be considered for risk groups in which it is critical not to miss TB, such as cases with smear and culture negative results. However, at a $1 \%$ prevalence the positive predictive value is $78 \%$ with an Xpert MTB/RIF test for diagnosing TB, while at $0.5 \%$ prevalence it is $64 \%$. [28]. At a prevalence less than $0.5 \%$, using chest radiography to screen for abnormalities suggestive of active TB followed by an Xpert MTB/RIF test yields 20\% false-positive cases (posi- 
tive predictive value $=79 \%$ at $0.5 \%$ prevalence) unless further diagnostic verification is done. Hence, culture or careful diagnostic assessment, or both, are necessary for persons who are positive by Xpert MTB/RIF testing, especially when the TB prevalence is less than $0.5 \%$. Immunodiagnostics of TB has also been shown to be useful in predicting active smear negative TB, especially in low incidence settings of TB [29-31]. Furthermore, an immunological test (such as the QFT test) is expected to be a stronger predictor of pulmonary TB because it is less likely to be affected by non-tuberculous mycobacteria infection and Bacillus Calmette-Gue'rin vaccination compared to the tuberculin skin test [31]. However, the QFT test is unable to differentiate between latent and active TB infection. Because of this, it is of limited value, although it is a significant predictor in the clinical diagnosis of active pulmonary TB.

WHO estimates that about one third of all incident cases of active TB are not properly diagnosed or receive care of questionable quality outside of national TB programs [32]. What seems clear from our study is that TB suspects without an initially confirmed diagnosis of TB are poorly served by current systems. Thus, echo a fundamental principle of TB control espoused by Piot more than 40 years ago, that a substantial proportion of TB cases occur among individuals who fail to access high quality diagnostic and treatment services [33, 34]. On the other hand, since early 2000, all surveys of TB prevalence done in countries with reasonably well performing national TB programs, have consistently demonstrated that the majority of undiagnosed cases of TB are sputum-smear negative. They have also demonstrated that more than $50 \%$ of those with prevalent bacteriologically confirmed TB do not report symptoms that correspond to the commonly used criteria for suspecting disease and prompting diagnostic investigation [35-37]. In our studies we showed that a higher proportion of clinical actions were omitted during diagnosis and monitoring of treatment response in smear-negative compared with smear-positive TB cases, despite the fact that the clinical actions are especially important for the former. Our findings add that care offered to smear-negative cases continues to be deviant from the standards, compared to smear-positive TB cases. However, this could be reduced if programme managers and policymakers, pay more attention to clinical measures related to care for smear-negative TB patients that are poorly followed compared to smearpositive TB cases. Therefore, we suggest that future quality improvement initiatives should focus on the role of real-time, clinic-level monitoring of smear negative TB suspect evaluation practices, in order to capture individual data on clinical measures related to care of smear-negative TB from every patient evaluated. Tools for electronic data collection, including mobile telephone based applications, are becoming readily available and can facilitate real-time monitoring and evaluation of TB care at the level of individual clinics. This approach deserves preference over existing monitoring and evaluation systems, since the system captures individual data on symptoms of TB from every patient evaluated in primary health clinics, permitting direct calculation of the numbers at risk for and suspected of TB, and longitudinal measurement of the quality of TB evaluation services for an individual clinic or provider. In a study by Davis et al, electronic monitoring 
and evaluation proved to be feasible, and this study demonstrated that modest improvements in individual clinic performance in assessing patients suspected of TB were associated with increases in TB case detection [38].

Screening is particularly appropriate to find TB patients who are non-symptomatic or only have vague symptoms that are unlikely to be recognized by the person with the disease during early stages [39]. Thus, the critical question is whether culture-negative TB suspects can be detected and treated early enough. Our finding is in agreement with a previous study that reported that predictive models based on clinical variables may not be useful to discriminate culture-negative TB, because of the variable presentations of symptoms [7]. Screening for active TB is one of several possible interventions that can improve early detection of all forms of TB, but providing better access to diagnostic tests that are more sensitive than smear microscopy is the first essential step. From the above studies it is not possible to confidently conclude whether predictive models contributed additional cases or only identified cases that would have been detected through passive case finding. For such an evaluation, a controlled design comparing areas that use or do not use such predictive models, is required. In addition, when there is little empirical evidence, mathematical modelling can help create scenarios to assess the possible impacts, but these scenarios require verification through clinical trials [40, 41]. However, without reliable parameters for the natural course of culture-negative TB, such models should be interpreted with caution.

It has been suggested that volatile metabolites from Mtb organisms may be exploited to diagnose TB rapidly and could be used as biomarkers of cure and relapse [42]. This is an area of much interest and may lead to a practical, non-invasive way of diagnosing TB at site of care. However, there is a lack of clinical studies describing the level of salivary NO in suspected TB patients in the diagnostic phase. Human mouth represents the environment with a constant supply of NO metabolites from L-arginine. The production of salivary NO metabolites, may be affected during nutritional deficiencies, a common condition in TB suspects $[43,44]$. The results of our findings support our hypothesis that, patients unable to respond with an effective production of salivary NO metabolites in response to $M t b$ infection are more likely to have TB. Our findings and previous studies suggest that the use of NO metabolites as a biomarker may have great value for screening of smear positive TB. However, there is the potential downside that this single biomarker is not necessarily TB-specific, a weakness that may be addressed by the identification of biosignatures-multiple signals driven by specific host-pathogen interaction $[45,46]$. Multiplex assessments have proven to have significant prognostic/diagnostic value in cancer [47, 48], HIV infection [49] and malaria [50]. Several recent studies suggest the same is true in TB, implying that highly multiplexed assays may be superior to single immune biomarkers. Furthermore, uncertainties exist for the use of these tests to diagnose infection status prior to initiation of therapy, the most important being the degree to which these tests can differentiate recent or progressive infection from a distant or latent infection. Inability to distinguish these two states will greatly reduce 
the potential for these tests to add value to clinical decision-making in TB-endemic regions, where a majority of adults would be expected to have been exposed to Mtb. There are many studies that suggest that the magnitude of the immune response reflects the magnitude of the bacterial load and two longitudinal studies in untreated contacts suggest that the highest responders are at greatly elevated risk of TB [51, 52], but these studies are relatively small and lack detailed sequential testing routines. Thus the kinetics and duration of the differences are unknown; however, the fact that the two studies produced similar results in very different populations and environments (Ethiopia and Germany) is encouraging. Studies to address the questions of remote versus recent infection, predictive value and optimization of the assays, and their usefulness in HIV infected patients should prove very valuable.

We must consider a few methodological issues when interpreting the results of our studies. First, in this study we evaluated the performance of predictors for estimating the probability of culture-negative TB when all versus only significant variables were considered among smear negative cases. Our approach is different from previous studies that based their analysis on combining only independent significant predictive variables into a decision model [53-57]. Information from a single predictor is often insufficient to provide reliable estimates of diagnostic probabilities or risks [58, 59]. However, we did not follow-up predictive variables (or clusters of variables) of culturenegative $T B$, progressing over time that may be useful in diagnostic decision making. Culture-negative TB patients often present early in the course of an illness when typical symptoms and signs are absent [60]. Therefore, as the condition evolves, clinicians may rely more on the assimilation of information gained over a period of time ('dynamic evidence', e.g. the addition of new features, the persistence or changes in the characteristics of previous problems) rather than the traditional static information we obtained in our study at one point [61]. Second, it is clear from our study, that we did not evaluate how often and to what extent uncertain diagnostic outcome is shared with patients during consultations. Failure to communicate uncertainty effectively can lead to patients failing to return until they are approaching death [62].

Operational research has been increasingly recognized as vital to the strengthening of health programmes. For example, the expanded Stop TB Strategy [63] explicitly includes operational research as one of the key components for successful TB programmes. A strong connection exists between good monitoring and evaluation of infectious-disease programmes and operational research [64]. Since, good quality data on cases and treatment outcomes can be used to do operational research, which in turn can help to improve the routine data collected in the field. Operational research means that the search for knowledge on interventions, strategies, or tools can enhance the quality, effectiveness, or coverage of programmes in which the research is being done [64].The importance of operational research is its ability to address and solve local problems in delivering quality health services. A necessary starting point is to identify the obstacles to providing high quality services, analyse why these obstacles occur and 
to adopt policies and practices to overcome them [65]. As such, operational research is important to initially obtain more data to build on knowledge already acquired [6-8] and then to work out how best to integrate TB suspect follow-up into routine care. If we do act to improve the follow-up and management of TB suspects with smear and culture negative results, how will we do this and how will we monitor it? Firstly, if the diagnosis is uncertain, that uncertainty should be communicated to the patient (or parent/carer), so that they are empowered to re-consult if necessary. In addition, if there is a recognized risk of deterioration or complications developing then a safety-net advice should include the specific clinical features (including red flags) that the patient (or parent/carer) should look out for. Secondly, we suggest that future research into quality of diagnostic care of culture-negative TB, should better adopt a study design that closely reflects its natural history. For example, three to four weekly re-assessments (if possible combined with symptom diaries) of patients would not only provide more useful 'dynamic' clinical information, but would also enable the study to assess more reliably the association between the clinical information and the eventual diagnosis. This pragmatic approach would be ideal, since clinicians in Ethiopia use all available information for each patient to determine whether TB is indeed present or absent in case of negative smear and/or culture test results [66]. Besides, there are a number of 'test of time' strategies, [67] which allow clinicians to deal with uncertain diagnostic outcome more effectively. Often these strategies 'buy time' for the condition to either resolve or declare itself more floridly and leads to explicit identification of patients with 'undifferentiated presentations' that can all help target antibiotics to those most likely to benefit. For example, safety-netting has been proposed as a method of reducing the risk of missing serious disease in patients with an 'uncertain' diagnostic outcome [68]. Research designed around the explicit identification of patients with 'undifferentiated presentations' would facilitate the evaluation of different strategies for programme managers to policy-makers and from medical personnel to handle this subgroup in clinical practice [69]. This would impact significantly on general practice, providing clinicians with a set of validated tools to confidently handle uncertain diagnostic outcome. 


\section{REFERENCE}

1. World Health Organization (2007) Improving the diagnosis and treatment of smear-negative pulmonary and extra-pulmonary tuberculosis among adults and adolescents: Recommendations for HIV-prevalent and resource-constrained settings.

2. Apers L, Wijarajah C, Mutsvangwa J, Chigara N, Mason P, et al. (2004) Accuracy of routine diagnosis of pulmonary tuberculosis in an area of high HIV prevalence. Int J Tuberc Lung Dis 8: 945-951. 17.

3. Munyati SS, Dhoba T, Makanza ED, Mungofa S, Wellington M, et al. (2005) Chronic cough in primary health care attendees, Harare, Zimbabwe: diagnosis and impact of HIV infection. Clin Infect Dis 40: 1818-1827. 18.

4. Mutetwa R, Boehme C, Dimairo M, Bandason T, Munyati SS, et al. (2009) Diagnostic accuracy of commercial urinary lipoarabinomannan detection in African tuberculosis suspects and patients. Int J Tuberc Lung Dis 13: 1253-1259.

5. McCowan C, Fahey T. Diagnosis and diagnostic testing in primary care. Br J Gen Pract 2006; 56(526): 323-324.

6. Hong Kong Chest Service/Tuberculosis Research Centre, Madras/British Medical Research Council A controlled trial of 2- month, 3-monrh and 12 month regimens of chemotherapy for sputum-smearnegative pulmonary tuberculosis. AmRev Respir Dis 1984; 130: 23-28.

7. Cowie RL, Langton ME, Escreet BC. The diagnosis of sputum smear- and sputum culture-negative pulmonary tuberculosis. S Afr MedJ 1985; 68:878

8. Nguyen M-VH, Jenny-Avital ER, Burger S, Leibert EM, Achkar JM (2015) Clinical and Radiographic Manifestations of Sputum Culture-Negative Pulmonary Tuberculosis. PLoS (10): e0140003. doi:10.1371/journal.pone.0140003

9. Conde MB, Melo FA, Marques AM, Cardoso NC, Pinheiro VG, Dalcin PT, et al.III Brazilian Thoracic Association Guidelines on tuberculosis. JBras Pneumol 2009, 35:1018-1048. PMID: 19918635

10. Cobo J, Oliva J, Asensio A, et al. Predicting tuberculosis among HIV infected patients admitted to hospital: comparison of a model with clinical judgment of infectious diseases specialists. Eur J Clin Microbiol Infect Dis 2001; 20:779-784

11. Siddiqi K, Lambert ML, Walley J. Clinical diagnosis of smear-negative pulmonary tuberculosis in lowincome countries: the current evidence. Lancet Infect Dis. 2003; 3:288-96.

12. Automated real-time nucleic acid amplification technology for rapid and simultaneous detection of tuberculosis and rifampicin resistance: Xpert MTB/RIF system. Policy statement. Geneva, World Health Organization, 2011 (WHO/HTM/TB/2011.4)

13. Kanaya AM, Glidden DV, Chambers HF. Identifying pulmonary tuberculosis in patients with negative sputum smear results. Chest. 2001; 120:349-55. 23.

14. Wisnivesky JP, Kaplan J, Henschke C, et al. Evaluation of clinical parameters to predict Mycobacterium tuberculosis in inpatients. Arch Intern Med. 2000; 160:2471-6. 24.

15. Nakanishi M, Demura Y, Ameshima S, et al. Utility of high resolution computed tomography for predicting risk of sputum smear-negative pulmonary tuberculosis. Eur J Radiol. 2010; 73: 545-50.

16. Lee HM, Shin JW, Kim JY, et al. HRCT and whole-blood interferon-gamma assay for the rapid diagnosis of smear-negative pulmonary tuberculosis. Respiration. 2010; 79:454-60.

17. Reitsma JB, Rutjes AW, Khan KS, Coomarasamy A, Bossuyt PM (2009) A review of solutions for diagnostic accuracy studies with an imperfect or missing reference standard. J Clin Epidemiol 62: 797-806.

18. Hadgu A, Dendukuri N, Hilden J (2005) Evaluation of nucleic acid amplification tests in the absence of a perfect gold-standard test: a review of the statistical and epidemiologic issues. Epidemiology 16: 604612.

19. Enarson D, Rieder HL, Arnadottir T, Trébucq A (2000) Management of Tuberculosis: A guide for Low Income Countries. Paris: International Union Against Tuberculosis and Lung Disease.

20. American Thoracic Society (2000) Diagnostic Standards and Classification of Tuberculosis in Adults and Children. Am J Respir Crit Care Med 161:1376-1395. 
21. World Health Organization (2003) Treatment of tuberculosis: guidelines for national programmes, 3rd ed, Geneva, World Health Organization. Available: http://whqlibdoc.who.int/hq/2003/WHO_CDS_TB_ 2003.313_eng.pdf. Accessed 2010 Nov 20

22. Chan $W$ et al. Bacteriological measures for the detection of cases of pulmonary tuberculosis. Bulletin of the World Health Organization, 1971, 45:551-558.

23. Levy H, Feldman C, Sacho H, van der Meulen H, Kallenbach J, Koornhof H: A reevaluation of sputum microscopy and culture in the diagnosis of pulmonary tuberculosis. Chest 1989, 95:1193-1197.

24. Githui W et al. Cohort study of HIV-positive and HIV-negative tuberculosis, Nairobi, Kenya: comparison of bacteriological results. Tubercle and Lung Disease, 1992, 73:203-209

25. Foundation for innovative new diagnostics (2009) Rapid scale up of integrated molecular diagnostic laboratories. Available: http://www.finddiagnostics.org/export/sites/default/resource-centre/presentations/ stop_tb_forum_mar09/rapid_scale_up_rio_molecular_2.pdf. Accessed 2010 Feb 8.

26. Kanaya AM, Glidden DV, Chambers HF. Identifying pulmonary tuberculosis in patients with negative sputum smear results. Chest. 2001; 120:349-55. 23.

27. Automated real-time nucleic acid amplification technology for rapid and simultaneous detection of tuberculosis and rifampicin resistance: Xpert MTB/RIF system. Policy statement. Geneva, World Health Organization, 2011 (WHO/HTM/TB/2011.4).

28. WHO 2013. Systematic screening for active tuberculosis: principles and recommendations. WHO/HTM/TB/2013.04

29. Wisnivesky JP, Kaplan J, Henschke C, et al. Evaluation of clinical parameters to predict Mycobacterium tuberculosis in inpatients. Arch Intern Med. 2000; 160:2471-6. 24.

30. Nakanishi M, Demura Y, Ameshima S, et al. Utility of high resolution computed tomography for predicting risk of sputum smear-negative pulmonary tuberculosis. Eur J Radiol. 2010; 73: 545-50.

31. Lee HM, Shin JW, Kim JY, et al. HRCT and whole-blood interferon-gamma assay for the rapid diagnosis of smear-negative pulmonary tuberculosis. Respiration. 2010; 79:454-60.

32. Global tuberculosis control 2011. Geneva, World Health Organization, 2012

33. Piot M. A simulation model of case finding and treatment in tuberculosis

34. Kemp J R, Mann G H, Nhlema Simwaka B, Salaniponi F M L, Squire S B. Can Malawi's poor afford free TB services? Patient and household costs associated with a TB diagnosis in Lilongwe. Bull World Health Organ 2006; 85: 580-585.

35. Hoa NB et al. A national survey of tuberculosis prevalence in Vietnam. Bulletin of the World Health Organization, 2010, 88:273-280.

36. National TB Prevalence Survey, 2002, Cambodia. Phnom Penh: Ministry of Health, 2002.

37. Ayles $\mathrm{H}$ et al. Prevalence of tuberculosis, HIV and respiratory symptoms in two Zambian communities: implications for tuberculosis control in the era of HIV. PLoS One 2009, 4:e5602 (doi:10.1371/journal. pone.0005602).

38. Davis, Katamba, Vasquez, et al.: Real-Time Monitoring of TB Diagnostic Service. Am J Respir Crit Care Med Vol 184. pp 362-367, 2011

39. World Health Organization 2013. Systematic screening for active tuberculosis: principles and recommendations. WHO/HTM/TB/2013.04

40. Murray CJL, Salomon JA. Modeling the impact of global tuberculosis control strategies. Proceedings of the National Academy of Sciences of the USA, 1998, 95:13881-13886.

41. Borgdorff MW, Floyd K, Broekmans J. Interventions to reduce tuberculosis mortality and transmission in low- and middle-income countries. Bulletin of the World Health Organization, 2002, 80:217-227.

42. Phillips M et al. (2007). Volatile biomarkers of pulmonary tuberculosis in the breath. Tuberculosis (Edinburgh, Scotland), 87(1):44-52.

43. Peters, W. H. and Lubs, H. (1987) Serum free amino acids of healthy males and pregnant women from Ethiopia. Die Nahrung 31, 923-5.

44. Zea, A. H., Culotta, K. S., Ali, J., Mason, C., Park, H. J., Zabaleta, J., Garcia, L. F., Ochoa, A. C. (2006) Decreased expression of CD3zeta and nuclear transcription factor kappa B in patients with pulmonary tuberculosis: potential mechanisms and reversibility with treatment. J Infect Dis 194, 1385-93 
45. Jacobsen $\mathrm{M}$ et al. (2008). Novel strategies to identify biomarkers in tuberculosis. Biological Chemistry, 389(5):487-495.

46. Walzl G et al. (2008). Biomarkers for TB treatment response: challenges and future strategies. The Journal of Infection, 57(2):103-109. Epub 2008 Jul 22.

47. Bullinger $L$ et al. (2004). Use of gene-expression profiling to identify prognostic subclasses in adult acute myeloid leukemia. The New England Journal of Medicine, 350(16):1605-1616.

48. Burczynski ME et al. (2005). Transcriptional profiles in peripheral blood mononuclear cells prognostic of clinical outcomes in patients with advanced renal cell carcinoma. Clinical Cancer Research, 11(3):11811189.

49. Ockenhouse CF et al. (2005). Functional genomic relationships in HIV-1 disease revealed by geneexpression profiling of primary human peripheral blood mononuclear cells. The Journal of Infectious Diseases, 191(12):2064-2074.

50. Griffiths MJ et al. (2005). Genomewide analysis of the host response to malaria in Kenyan children. The Journal of Infectious Diseases, 191(10):1599-1611.

51. Diel R et al. (2008). Predictive value of a whole blood IFN-gamma assay for the development of active tuberculosis disease after recent infection with Mycobacterium tuberculosis. American Journal of Respiratory and Critical Care Medicine, 177(10):1164-1170

52. Doherty TM et al. (2002). Immune responses to the Mycobacterium tuberculosis-specific antigen ESAT-6 signal subclinical infection among contacts of tuberculosis patients. Journal of Clinical Microbiology, 40(2):704-706.

53. Scott B, Schmid M, Nettleman MD. Early identification and isolation of inpatients at high risk for tuberculosis. Arch Intern Med 1994; 154:326-330

54. Kara S. Rakoczy, MD; Stuart H. Cohen, MD; Hien H. Nguyen, MD. Derivation and Validation of a Clinical Prediction Score for Isolation of Inpatients With Suspected Pulmonary Tuberculosis. Infect Control Hosp Epidemiol 2008; 29:927-932

55. Wisnivesky J, Henschke C, Balentine J, Willner C, Deloire AM, McGinn TG. Prospective validation of a prediction model for isolating inpatients with suspected pulmonary tuberculosis. Arch Intern Med 2005; 165:453-457

56. Pegues C, Johnson DC, Pegues DA. Implementation and evaluation of an algorithm for isolation of patients with suspected pulmonary tuberculosis. Infect Control Hosp Epidemiol 1996; 17:412-418.

57. Bock NN, McGowan JE, Ahn J. Clinical predictors of tuberculosis as a guide for a respiratory isolation policy. Am J Respir Crit Care Med 1996; 154:1468-1472

58. Riley RD, Hayden JA, Steyerberg EW, Moons KG, Abrams K, Kyzas PA, et al; PROGRESS Group. Prognosis Research Strategy (PROGRESS) 2: prognostic factor research. PLoS Med. 2013; 10: e1001380. [PMID: 23393429]

59. Collins GS, Altman DG. Identifying patients with undetected renal tract cancer in primary care: an independent and external validation of QCancer $^{\circledR}$ (Renal) prediction model. Cancer Epidemiol. 2013; 37:11520. [PMID: 23280341]

60. Centers for Disease Control and Prevention. Reported tuberculosis in the United States, 2013. Atlanta, GA: Centers for Disease Control and Prevention, Services UDoHaH; 2013 May8, 2015. ReportNo.

61. Summerton N. Diagnosis and general practice. Br J Gen Pract 2000;50(461):995-1000

62. Pearson GA(Ed).Confidential Enquiry into Maternal and Child Health. Why Children Die: A Pilot Study 2006; England (Southwest, North East \&West Midlands), Wales and Northern Ireland. London: CEMACH, 2008.

63. The Stop TB Strategy, World Health Organization. 2006. http://whqlibdoc.who.int/hq/2006/ WHO_HTM_STB_2006.368_eng.pdf. [Accessed 06 March 2013]

64. Zachariah R, Harries D, Ishikawa $\mathrm{N}$ et al . Operational research in low income countries: what, why and how? Lancet Infect Dis 2009; 9: 711-717

65. Edginton ME, Wong ML, Hodkinson HJ. Tuberculosis at Chris Hani Baragwanath Hospital: an intervention to improve patient referrals to district clinics. Int J Tuberc Lung Dis 2006; 10: 10181022. 


\section{Chapter 6}

66. Mala G, Moser A, Dinant GJ and Spigt M. Why tuberculosis service providers do not follow treatment guide-line in Ethiopia: a qualitative study. J Eval Clin Pract 2014; 20: 88-93

67. Heneghan C, Glasziou P, Thompson M, et al. Diagnostic strategies used in primary care. BMJ 2009; 338: b946.

68. Almond S, Mant D, Thompson M. Diagnostic safety-netting. Br J Gen Pract 2009; 59(568): 872-874.

69. Green C, Holden J. Diagnostic uncertainty in general practice. Eur J Gen Pract 2003; 9(1): 13-15. 
Summary 

The studies presented in this thesis were focused on the quality of diagnostic care provided to suspected tuberculosis (TB) patients and centre on four principle investigations. The central aim of the studies presented in Chapters 2 and 3 was to gain understanding of the current quality of TB care and reasons for not following the guidelines. The overarching aim of the study presented in Chapter 4 is to develop strategies to diagnose TB in patients whose initial tests are negative for smears. A prospective study to determine the predictors of culture-negative TB among smear negative TB cases is described. Finally, in Chapter 5 we explore the level of salivary NO and its metabolites as a potential biomarker to detect the earliest and most difficult to diagnose TB cases. In the final chapter of this thesis, Chapter6, we presented the overall major findings, methodological considerations, and general conclusion of the studies.

In Chapter 1 of this thesis, we summarize the result of a literature review related to research questions regarding the quality of diagnostic care provided to suspected TB patients. In its Global TB Report 2013, the WHO highlighted detection of missed cases as a priority action to reach 2015 global targets. The estimated TB case detection rate (percentage of detected cases among the estimated number of incident cases) in Ethiopia has been consistently low; the WHO estimate is $64 \%$ for all forms. In a recent Ethiopian national population based survey in 2011, the prevalence of bacteriologicallyconfirmed TB (smear and/or culture positive) for persons aged 15 years and above was 277 per 100 000. Besides, reports from previous studies, showing a high proportion of TB among young persons, suggests that TB is circulating in the community and that there is a need for more efforts to limit the spread of TB disease.

Current TB control strategies in many countries depend on care that is provided to persons who have symptoms of TB and/or those who have the disease. In most highburden countries, TB is still diagnosed using tools such as direct sputum microscopy and chest radiographs. When applied to resource-limited settings, these methods suffer from significant limitations; accuracy is suboptimal and fails to diagnose one-third to half of all TB patients. To improve the accuracy of diagnostic work-up several new diagnostic tests have been suggested. However, no single parameter has gained undisputed acceptance. Predictive models for the diagnosis of TB provide a useful framework for systematization of the diagnostic approach and are able to standardize data collection from clinicians. These models could be used to identify patients at very high risk of TB that may require further diagnostic tests after the results of a negative smear test. Besides, further research is necessary to determine the usefulness of these prediction models to establish the exact causes of false smear negative TB (patients that have TB, but have a negative smear test). Furthermore, substantial effort has been devoted to improving physician compliance with evidence-based guidelines. Studies of the quality of TB evaluation (i.e. diagnostic workup of patients with symptoms suggestive of TB) in high burden countries have generally shown poor adherence to international or national guidelines. While previous research has assessed barriers patients face in accessing primary care centers that provide TB diagnostic services, less is known about barriers 
providers in these settings face in adhering to guidelines for evaluating patients for TB. We identified diagnostic guideline adherence and professional practice, as relevant elements of the under-emphasized theme in quality of TB care.

The aim of the studyreportedinChapter2wasto determine quality of diagnosis and monitoring of treatment response of patients with smear-negative TB compared with smear-positive cases. A retrospective review of medical records of newly diagnosed TB cases was performed and we descriptively analysed the proportion of TB cases managed according to ISTC protocol. Our findings show poor standardized clinical care offered to smear-negative TB cases compared to smear-positive TB cases. This could be due to the assumption that the prognosis for smear-negative cases is better than for smear-positive cases. However, this assumption can only be valid if microscopy procedures are followed completely and reliably. Therefore, there is a need to explore the reasons behind poor standardized clinical work-up in smear-negative compared to smear-positive TB cases.

The research aim of the second study, described in Chapter 3, was to explore TB service providers' reasons for not complying with TB treatment guidelines at health facilities in Ethiopia. A descriptive qualitative design was used. Focus group discussions (FGD) were held with 39 service providers involved in TB care. A questioning route for the FGDs was prepared, with open-ended questions, probes and prompts. The questions were based on information from the current literature (sensitizing concepts), and had been reviewed by stakeholders and adapted accordingly. The topics were quality deficits, compliance with TB guidelines and information transfer. For the data analysis we used the qualitative content analysis approach based on the constant comparison method. Codes and categories emerged inductively from the data through careful examination by the researcher. The main reasons for non-compliance with TB treatment guidelines were (1) insufficient diagnostic modalities, limiting the capacity to correctly diagnose TB and provide the right regimen; (2) ambiguity in guideline recommendations especially for specific sub-groups of patients, making it difficult to prioritize them to the right regimen; and (3) poor documentation concerning referred patients, with no appropriate examination and difficulty obtaining information from previously treated patients. Our findings offer real-life examples of what is needed to customize TB guidelines to local contexts of healthcare in order to improve compliance. We recommend developing intervention strategies that are not only tailored to average patients. Consideration should be given to specific sub-groups as an important influence on guideline compliance.

Despite the considerable incidence of culture-negative TB, little is known about this specific group's clinical manifestations compared to culture-positive TB among smear negative cases. In Chapter 4, we hypothesize that culture-negative TB cases may present with no significant differences in clinical and radiographic abnormalities when compared to those cases with a culture-positive and smear-negative result. A prospective evaluation study was designed to determine the predictive ability of routine diagnostic tests and clinical symptoms. While application of predictive modelling in patients 
with clinical suspicion of TB has been described before, previous studies based their analysis on combining only independent significant predictive variables into a decision model. Therefore, in this study, we evaluated the performance of predictive models when all and only significant variables were considered for estimating the probability of TB; (1) among all clinical suspects and (2) among smear negative cases. Employing all variables and only independent significant ones into a regression model, failed to discriminate between culture-negative and culture-positive among smear-negative TB cases, thereby supporting our hypothesis that among smear-negative cases, culturenegative TB may present with no differences in clinical and radiographic abnormalities compared to those with culture-positive TB. This finding emphasizes that medical providers should neither rely on culture as the gold standard tests for TB, nor expect a clinical presentation with the symptoms characteristic of advanced TB in order to diagnose and initiate treatment in culture-negative TB. Furthermore, our findings suggest that independent significant variables (positive sputum smear and chest radiography compatible with TB) remain critical elements in prediction of culture-positive TB among patients with clinical suspicion.

In the Global TB Report 2014, the WHO declared that "one of the most urgently needed tests is a rapid biomarker-based test that can diagnose TB". Ideally, such TB biomarker should be able to detect the earliest and most difficult to diagnose TB cases, and should be suitable for use in resource-limited settings. A series of reviews has shown that biomarkers such as NO have the potential for screening TB suspects. Human mouth represents the environment with a constant supply of NO metabolites from Larginine. The production of stable salivary NO metabolites, may be affected during nutritional deficiencies, a common condition in TB suspects. Several studies reported that patients with active TB are more likely to experience wasting, and have a lower body mass index (BMI) than healthy individuals. Therefore, it is hypothesized that patients unable to respond with an effective production of salivary NO metabolites in response to $M t b$ infection, are more likely to have severe (commonly seen as wasting in TB suspects) and more infectious forms of TB. In Chapter 5, an exploratory study to compare the salivary NO levels in confirmed and unconfirmed suspected TB patients by sputum smear microscopy, chest radiography and molecular GeneXpert tests, is summarized. The objective of the study was to examine the diagnostic value of the level of salivary NO metabolites, in order to assess microbiological outcome (infectiousness), and its association with the severity of the disease (commonly seen as wasting) in TB suspects. The results of our findings support our hypothesis that patients unable to respond with an effective production of salivary NO metabolites in response to Mtb infection, are more likely to be associated with increased risk of acquiring TB. Interestingly, the results could not support our second hypothesis that levels of salivary NO could be associated with the severity of the TB. This finding is also in line with the study of Idh et al., in which they neither found an association between BMI and NO levels at 
treatment initiation. However, larger clinical studies, including long term clinical follow up will be essential to test this hypothesis thoroughly.

The findings in this studies presented in this thesis suggest a number of avenues for further research and specific public policy implications for professional practice. Our findings indicate that the degree of trust that TB service providers had in the diagnostic test results had a great impact on clinician's compliance with the guidelines. Thus, addressing their negative perception of sputum smear microscopy may be just as important as scaling up the diagnostic test. Consideration should be given to specific sub-groups (smear and culture negative TB suspects) as an important influence on guideline compliance. However, in our study a critical question was whether smear and culture negative patients can be detected early by predictive modelling. Our finding is in agreement with a previous study that reported that predictive models based on clinical variables may not be useful to discriminate culture-negative TB, because of the variable presentations of symptoms. These symptoms are somewhat ubiquitous in a clinical setting, thereby nonspecific, and could possibly indicate a multitude of other illnesses. Although the coexistence of illness in smear and culture negative TB is problematic in itself, a fundamental challenge arises when clinical responses to this uncertainty are guided by the single disease paradigm that dominates the existing health care system. This paradigm leads to 'incomplete assessment of diagnostic outcome and failure to modify the clinical approach accordingly'. One possible direction for further research is the use of a diagnostic panel, i.e. a group of experts who assesses the results from all available relevant patient data to reach a final diagnosis in each patient. However, few studies have systematically examined the accuracy of TB diagnosis by such a panel and the type of diagnostic evidence available. Besides, approaches that combine clinical judgment based on all available information with additional value of new diagnostic tests have been proposed, but rarely addressed. Furthermore, we suggest that future quality improvement initiatives should focus on the role for real-time, clinic-level monitoring of smear negative TB suspect evaluation practices, in order to capture individual data on clinical measures related to care of smear-negative TB from every patient evaluated. 
Valorisation 



\section{VALORISATION: IMPLICATION FOR FUTURE RESEARCH AND PRACTICE}

In this section, I consider possible implications, both for the theoretical development of research on quality of TB diagnostic care and the specific public policy implications for professional practice in patients with suspected TB in resource poor setting. The findings in this study suggest a number of avenues for further research. Firstly, I recognize that by situating this research in Ethiopia context, the generalizability of my findings outside the resource constrained setting is limited. Although recent WHO report and others have shown that clinicians often deviate from internationally recommended TB management, our findings add that care offered to smear-negative cases continues to be deviant from the standards, compared to smear-positive TB cases. This then raises the question of the perceived habitual bias by TB healthcare providers, who apparently still consider managing smear-positive cases to be the most effective strategy to curb the TB epidemic. This treatment adherence gap would be significantly closed if strategies that increase compliance to ISTC are identified by programme managers and policymakers in hospitals that best meet the desired standards. Therefore, we suggest that future quality improvement initiatives should focus on the role for real-time, clinic-level monitoring of smear negative TB suspect evaluation practices, in order to capture individual data on clinical measures related to care of smear-negative TB from every patient evaluated.

Less well understood are the determinants of provider adherence to TB treatment guidelines. In particular, there is increasing recognition that guideline implementation is heavily dependent on provider behaviour and in order to improve the quality of diagnostic care, understanding and subsequently changing provider behaviour is required. Our findings indicate that the degree of trust that TB service providers had in the diagnostic test results had a great impact on compliance with the guidelines. Thus, addressing their negative perception of sputum smear microscopy may be just as important as scaling up the diagnostic test. Furthermore, there was dilemma among TB service providers about relying only on sputum microscopy in passive case finding. The underlying conflict was a lack of alternative diagnostic modalities when the initial test result does not correspond with the patient's clinical presentation. A better strategy to retest those patients whose initial tests are negative in settings where TB is frequently missed may improve compliance with the guideline.

A recent review of the TB guidelines showed that the overall methodological quality of the guideline was low. Several domains of the guidelines, such as applicability to specific local situations, contributed to their poor performance. Our findings offer reallife examples of what is needed to customize TB guidelines to local contexts of healthcare in order to improve compliance. We recommend, more studies in other regions and across a wider health facility spectrum to generalize a whole picture of nonadherence in Ethiopia and develop appropriate strategies that influence their compliance with the guidelines. With the important limitation of this study described above in 
mind, further studies are now needed to develop appropriate interventions aimed at addressing the critical factors suggested by this study, and to test these systematically. Consideration should be given to specific sub-groups (smear and culture negative TB suspects) as an important influence on guideline compliance.

Numerous decisions are made by care providers, on the basis of an estimated probability that a specific disease or condition is present in an individual. In the diagnostic setting, the probability estimates are commonly based on combining information from multiple predictors observed or measured from an individual. While application of predictive modelling in patients with clinical suspicion of TB has been described before, these studies usually combined only independent significant predictive variables into the decision models. In addition, information from a single predictor is often insufficient to provide reliable estimates of diagnostic probabilities or risks. However, in this study the critical question is whether patients with smear and culture negative result can be detected and treated early by predictive model. Our finding is in agreement with a previous study that reported that predictive models based on clinical variables may not be useful to discriminate culture-negative TB, because of the variable presentations of symptoms. Therefore, screening for active TB is one of several possible interventions that can improve early detection of all forms of $\mathrm{TB}$, but providing better access to diagnostic tests that are more sensitive than smear microscopy is the first essential step. In addition, sound training in threshold principles and providing tools to correctly assess patients with smear and culture negative results might help in making a better diagnostic decision in resource poor settings.

There is an absolute need to find biomarkers that can distinguish between individuals infected having the highest risk of developing TB (susceptibility) and those who have the highest probability of resisting development of the disease (protection). This would significantly help the control programme to break the chain of transmission of the disease in the long run. We are also in need of validated biomarkers that can be translated as point-of-care assays for early diagnosis and treatment of active TB patients in highincidence countries. The results of our findings support our hypothesis that, patients unable to respond with an effective production of salivary NO metabolites in response to Mtb infection, are more likely to be associated with increased risk of acquiring TB. Our findings and previous studies suggest that the use of NO metabolites as a biomarker may have great value for screening of smear positive TB. Will it be possible to learn from this naturally induced host response and better understand those mechanisms that control infection and exploit their value as potential indicators of protection? There are many studies that suggest that the magnitude of the immune response reflects the magnitude of the bacterial load and two longitudinal studies in untreated contacts suggest that the highest responders are at greatly elevated risk of TB, but these studies are relatively small and lack detailed sequential testing routines. Thus the kinetics and duration of the differences are unknown; however, the fact that the two studies produced similar results in very different populations and environments (Ethio- 
pia and Germany) is encouraging. Studies to address the questions of remote versus recent infection, predictive value and optimization of the assays, and their usefulness in HIV infected patients should prove very valuable. 

Acknowledgements 



\section{ACKNOWLEDGEMENTS}

I am sincerely grateful to everyone who has contributed to this thesis, and I would especially like to mention the following people:

To my promoter, Geert-Jan, for welcoming me to the department despite my more adventurous than scientific attitude (I hope the latter has improved a bit), for trust in independence and support when asked for (from wherever in the world you happened to be) and encouragement through the five-year process, even if we never came to a final agreement on the use of commas.

To my co-promoter, Mark, who has not only been a dedicated mentor, but a friend and a model to follow. Our interactions were always quite informal and friendly. Thank you for your never-ending energy, enthusiasm and for your support both when things go well and when life is tough. I consider myself quite fortunate to have had such an understanding and caring adviser, throughout the course of my studies.

\section{On a personal note,}

A special thank you to my friends Zerihun, Loko, Hayelom and Ms. Lori Mees (for their passion and support from the beginning to the end of my studies); Marlees, Hans, Riet, and Maud (who were hospitable as always whenever I visited their homes in Maastricht during my studies). My apologies to those I neglected to mention.

I would especially like to thank my family members who patiently supported me and persevered with my rolling deadline of thesis submission. I must specially mention my wife Jacquelove, who obviously had to endure a lot more of my own pressures. To my daughters Gracey and Ricci, for illuminating my life and accompanying me in spirit in the adventure of leaving our Kenyan life for in order to complete this PhD.

I would like to give all credit of this thesis to my mother, Helida and my grandfather, Odongo Mala who brought me up with love and gave me all good things. Both of them passed on when I was away from home, completing my studies. My commitment to get a PhD for them motivated and helped me to overcome obstacles and finish this thesis.

This study was funded by Nuffic Fellowship Program that promote capacity building within organizations in 51 countries by providing fellowships for training and education for professionals.

With many thanks!!!!!!

Mala George 

Biography 



\section{GEORGE MALA OTIENO}

1. Personal details

$\begin{array}{ll}\text { Date of Birth: } & 15^{\text {th }} \text { June } 1974 \\ \text { Nationality: } & \text { Kenyan } \\ \text { Marital status: } & \text { Married } \\ \text { Current Location: } & \text { Mekelle, Ethiopia } \\ \text { Current Position: } & \text { Expatriate lecturer, Mekelle University } \\ \text { Home Address: } & \text { C/O Jacqueline Sungu Matendechere } \\ & \text { P.O.Box 2642, Bungoma, Kenya. }\end{array}$

2. Professional qualification

2011- 2017

PhD Candidate at University of Maastricht, department of Family Medicine and Primary Healthcare

Degree Expected: $\quad$ PhD (spring 2017)

Thesis Title: Diagnostics evaluation of smear-negative Tuberculosis in a Resource Poor Setting.

Institution: Maastricht University, Dept of Family Medicine; Mekelle University, Dept. of Biomedical Sciences, Mekelle, Ethiopia

Funded by: $\quad$ Nuffic, PhD fellowships program

2000-2005: $\quad$ The University of Nairobi, Department of Biochemistry

Degree: MSc. Biochemistry

Thesis Title: $\quad$ Elution, purification and partial characterization of IgG bound to normal and pathological human placentae.

1995-2000:

Egerton University- Kenya, Faculty of Sciences

Degree:

BSc. (Biochemistry/Zoology) 


\begin{tabular}{llll}
\hline Date & Department & Organisation & Position \\
\hline $\begin{array}{l}\text { Nov 2004-August } \\
2006\end{array}$ & Dept. of Biochemistry & University of Nairobi & Graduate \\
Sept 2006-To Date & $\begin{array}{l}\text { Dept. of Medical } \\
\text { Biochemistry }\end{array}$ & Mekelle University & Lecturer \\
Jan 2011-2017 & $\begin{array}{l}\text { Dept. of Family } \\
\text { Medicine }\end{array}$ & Maastricht University & PhD candidate \\
\hline
\end{tabular}

4. University Teaching Experience (Mekelle University-Ethiopia 2006-Date)

\begin{tabular}{|c|c|c|c|}
\hline Department & Programs & $\begin{array}{l}\text { Courses } \\
\text { offered }\end{array}$ & Specisspecial modules offered \\
\hline $\begin{array}{l}\text { Depts. of Health's } \\
\text { and Medicine }\end{array}$ & $\begin{array}{l}\text { MB.Ch B I, BDS I, } \\
\text { B.Pharm I, BScN } \\
\text { I, BSc Public } \\
\text { health (Regular } \\
\text { programs) }\end{array}$ & $\begin{array}{l}\text { Medical } \\
\text { Biochemistry }\end{array}$ & $\begin{array}{l}\text { Molecular medicine, Biochemical } \\
\text { pharmacology, Nutritional } \\
\text { Biochemistry, Neurochemistry, } \\
\text { Clinical Immunology }\end{array}$ \\
\hline Depts. of Nursing & $\begin{array}{l}\text { Cataract, } \\
\text { Neonatal and } \\
\text { Emergency, } \\
\text { Nursing (Special } \\
\text { programs) }\end{array}$ & $\begin{array}{l}\text { Medical } \\
\text { Biochemistry }\end{array}$ & $\begin{array}{l}\text { Biochemistry of the eye, In born } \\
\text { errors of metabolism, Acid-Base } \\
\text { balance, electrolyte and Paediatric } \\
\text { Biochemistry }\end{array}$ \\
\hline $\begin{array}{l}\text { Dept of Public } \\
\text { Health }\end{array}$ & $\begin{array}{l}\text { MSc. Public } \\
\text { Health }\end{array}$ & $\begin{array}{l}\text { Medical } \\
\text { Biochemistry } \\
\text { \& research } \\
\text { supervision }\end{array}$ & $\begin{array}{l}\text { Nutritional Biochemistry, research } \\
\text { supervision in TB diagnostic and } \\
\text { quality of care }\end{array}$ \\
\hline Dept of Density & $\begin{array}{l}\text { MSc. } \\
\text { Orthodontics }\end{array}$ & $\begin{array}{l}\text { Medical } \\
\text { Biochemistry }\end{array}$ & $\begin{array}{l}\text { Molecular genetics (Principles of } \\
\text { Orofacial and Malocclusion) }\end{array}$ \\
\hline
\end{tabular}

\section{Other responsibilities}

1. Head of Clinical Biochemistry course unit (2006-2013)

2. Mentor for medical students (mentoring students throughout the first year of their bachelor in medicine)

3. External Oral examiner for Medical Biochemistry to Preclinical students (Addis Ababa, Hawassa, Bahidar and Gonder Universities in Ethiopia).

\section{Current Research Interest}

1. Endocrine disruptors in reproductive health and metabolism 
2. The role of biomarker for screening and diagnostics of suspected tuberculosis (TB) patients and relation to treatment outcome.

3. Impact of cholesterol and cathelicidin LL-37 levels on Host Immunity to Mycobacterium Tuberculosis and Response to Treatment

4. The protective role of glucose-6-phosphate dehydrogenase (G-6-PDH) activity against risk factors of cardiovascular diseases

\section{List of publications and submitted Manuscripts}

1. Omwandho C. A, Gruessner E.E, Falconer J, Tumbo-Oeri A.G, Mala G, Mecha E, Tinnerberg H.R, Roberts T.K 2006. Ovine placental eluate immunoglobulins recognize isologous and third party acid treated trophoblast microvesicles antigens in Vitro.Jounarls of South African Veterinary Association 77(1):24-27.

2. Mala G, Spigt M, Gidding LG, Blanco R and Dinant GJ. Quality of diagnosis and monitoring of tuberculosis in Northern Ethiopia: medical records-based retrospective study: Tropical Doctor 2015; 45(4):214-20.

3. Mala G, Moser A, Dinant GJ, and Spigt M. Why tuberculosis service providers do not follow treatment guideline in Ethiopia: a qualitative study. J Eval Clin Pract 2013.

4. Mala G, Dinant GJ, Berhe S, Kiti LA and Spigt M. Predicting smear- and culturenegative tuberculosis with routinely available diagnostic tools in Ethiopia. Submitted Manuscript

5. Mala G, Spigt M, Berhe S, Tesfay H and Dinant GJ. Dealing with diagnostic uncertainty in suspected Tuberculosis (TB) patients with a negative smear-and culture results: A consensus diagnostic evaluation study. Submitted Manuscript

6. Parsons B J, Mala G, Dinant GJ, Ngeny C and Spigt M. Exploratory analyses of the level of salivary Nitric Oxide (NO) metabolites and microbiological outcome in suspected Tuberculosis (TB) patients. Submitted Manuscript

\section{Referees}

- Dr. Mark Spigt (PhD), Maastricht University / CAPHRI School for public Health and Primary Care, Department of general practice

Email:m.spigt@maastrichtuniversity.nl

- Prof. Tsehaye Asmelash (PhD), Vice-President for Research and Community service, Mekelle University

Email: tsehaye93@yahoo.com,

Mobile: 09128956149

- Dr. Loko Abraham, Dean College of Health Sciences, Mekelle University-Ethiopia.

Email: lokab77@yahoo.com

Mobile: +251911657510 
